\title{
Dipole-Dipole Interactions in a Cold Rydberg Gas
}

Brian Gordon Richards

Virginia Beach, VA

B.S., The College of William and Mary, 2010

\begin{abstract}
A Dissertation presented to the Graduate Faculty of the University of Virginia in Candidacy for the Degree of

Doctor of Philosophy
\end{abstract}

Department of Physics

University of Virginia

August, 2017 


\section{Abstract}

We investigate the strength and effects of dipole-dipole interactions in a cold Rydberg atom system. Our experiments explore the absence of collective decay due to dipole-dipole interactions, characterize dipole-dipole interactions and their dependence on Rydberg atom density and atomic motion, and explore possible methods to control atom separation using dipole-dipole interactions.

We study the decay of Rydberg atoms in a magneto-optical trap. The absence of collective decay or superradiant decay is attributed to variations in transition energies within the atom sample. These variations are dominated by inhomogeneities due to dipoledipole exchange interactions for initial $s$ states and by a combination of dipole-dipole and electric field inhomogeneities for initial $p$ states.

We characterize the strength of the dipole-dipole resonant energy transfer reaction $32 p 32 p \leftrightarrow 32 s 33 s$ by measuring population transfer to the $32 s 33 s$ pair state as a function of electronic energy difference between the initial and final atom-pair states. We obtain resonance line shapes and widths that agree with a randomly distributed, nearest-neighbor model. We also use the line shapes to estimate the electric field inhomogeneity.

We explore two possible methods of controlling atom separation using dipole-dipole 
interactions. The first uses a chirped-frequency control laser to push closely separated atoms apart in a fully formed magneto-optical trap. The second uses a fixed-frequency control laser to prevent atoms from approaching past a minimum separation as the trap is formed. While atomic separation control was not observed in these measurements, we present several improvements for future experiments. 


\section{Acknowledgments}

As with all dissertations, it took a large cast of characters to bring this one to fruition. There are far too many people to thank for the last seven years of my graduate school career and the last twenty-three years of my education. Knowing that I will be unable to encompass everyone, I must try to acknowledge those without whom this work would not have been possible.

First, I want to thank my family for all of their support over the years. Thank you Mom and Dad for your encouragement, stories of your own struggles, and for lending an ear when I needed it most. You two taught me how to succeed and have always provided me a space to seek out my own interests.

I want to thank my advisor, Professor Robert Jones, for his support and mentorship over the last six years. Your wide-ranging knowledge has proven invaluable in making me a better researcher. Whether it involved helping me understand the implications of my measurements, helping me grasp how fundamental physics applies to my research, teaching me about vacuum systems, or helping me weld, I would not have made it this far without you. Though I may struggle at times with the lessons you have taught me, they have helped mold me into a better physicist.

Thank you to Professors Stefan Baeßler, Lou Bloomfield, and Tom Gallagher for serving on my research committee. Your presence on my research committee has helped me refine my research and think deeper about the physics of my project. Thank you to the members of my defense committee, Professors Tom Gallagher, Cass Sackett, and Kevin Lehmann, for taking the time to read this dissertation and help me along the path to completion.

The help of my former lab mates, Dr. Tao Zhou, Dr. Sha Li, and Dr. Kisra Egodapitiya was instrumental to my success. Thank you Tao for teaching me how to run a cold atom experiment and for the many hours of assistance in getting my own experiments to work. Thank you Sha and Kisra for all your many discussions and contributions. I also want to

thank my current lab mates Chengxing He, Sanjay Khatri, Mark Gordon, and Dr. Péter 
Sandor for their many discussions and refinements of my work.

I want to thank Evan Kim and Lauren Levac. Without you two I likely would not have made it through my first two years. While I have missed your company for the last few years, I am happy you both have moved on to bigger and better things. Eric Magnuson, thank you for providing me with a sanity check whenever I go too deeply down a rabbit hole. Your insights helped propel these projects forward and I will be forever grateful for our lunchtime physics discussions. I also want to thank the amazing staff of the UVA Physics department, particularly Dawn Shifflett, who kept me going through the hardest parts of the program.

Thank you to the College of William and Mary Physics department staff and faculty, particularly Professor Seth Aubin. Seth, you were the person who first introduced me to atomic physics research and cold atoms. Without your guidance, I would never have applied to UVA for graduate school. Thank you for your efforts and for making me feel welcome when I have returned to W\&M. I also want to thank my undergraduate classmates, Virginia Forstall, Colton O'Connor, Francesca Fornasini, Ryan Zielinski, and Jordan Gates, who helped me get through the tough times in classes.

I want to extend thanks to my mentors at the National Institute of Standard and Technology during my time there as a Summer Undergraduate Research Fellow. Thank you Dr. Donald Windover, Dr. Joshua Pomeroy, and Dr. Katherine Mullen for giving me skills that helped get me through the beginning of graduate school. I want to thank Dr. Mullen in particular for introducing me to the wonderful world of R. The knowledge I gained during my summer sharing an office with you has proven invaluable in changing the way I think about, process, and visualize data.

During my time in Charlottesville, I was fortunate to live with a great group of people. Thank you Kyle Martin, Franny Yackso, Peter Bonventre, and Scott Bailey for your constant support both during and after our time living together. I also want to thank Ariel Kahrl and Karen Bailey for being near constant fixtures within our house. The six of you are like family to me.

I also want to thank the following people for their support and contribution to my work: Marc Presler, Mary Ingram, Anne Williams, Laura Matthews, the members of Swing Cville, the UVA R UseR group, and my friends in the blues dancing and Lindy Hop communities across the country.

Finally I want to thank Katie Dillon, whose encouragement over the last year has helped fill me with determination. 


\section{Contents}

1 Introduction 1

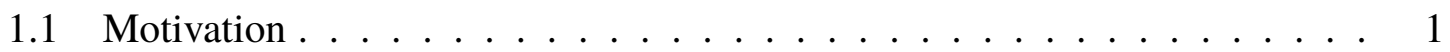

1.2 Atomic Units . . . . . . . . . . . . . . . . . . . 2

1.3 Rydberg Atoms . . . . . . . . . . . . . . . . . . . 3

1.4 Dipole-Dipole Interactions and Resonant Energy Transfer . . . . . . . . . . 4

1.5 Dissertation Structure . . . . . . . . . . . . . . . . 6

2 Experimental Setup and Equipment $\quad 8$

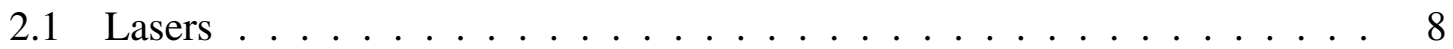

2.1 .1 Diode Lasers . . . . . . . . . . . . . . . . 8

2.1.2 Nd:YAG Laser . . . . . . . . . . . . . . . . . . 15

2.1.3 Dye Amplifier . . . . . . . . . . . . . . . . . . 16

2.2 Magneto-Optical Trap $($ MOT) . . . . . . . . . . . . . . . 18

2.2.1 Theory of Operation ..................... 18

2.2.2 Implementation . . . . . . . . . . . . . . . . 22

2.2.3 Characterization, Updates, and Modifications . . . . . . . . . . 28 


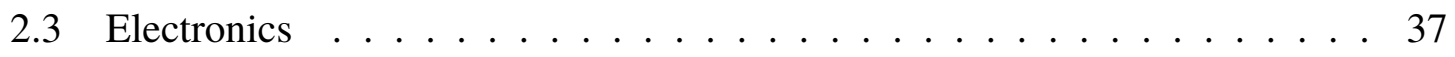

2.3.1 Field Ionization . . . . . . . . . . . . . . . . 37

2.3.2 Micro-Channel Plate (MCP) Detector . . . . . . . . . . . . . 38

2.3.3 Electric Fields . . . . . . . . . . . . . . . 38

2.3.4 Delay Generators . . . . . . . . . . . . . . . . . . . 39

2.3.5 Frequency Stabilization . . . . . . . . . . . . . . 40

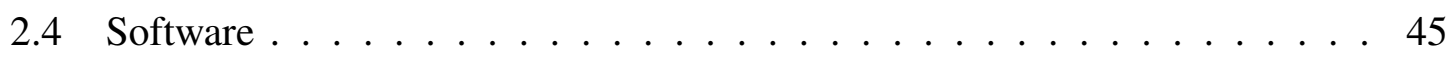

2.4.1 Data Acquisition ................... 45

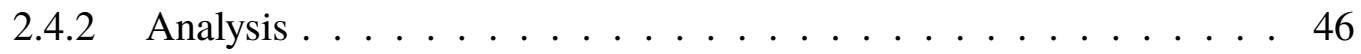

3 Absence of Collective Decay in a Cold Rydberg Gas 48

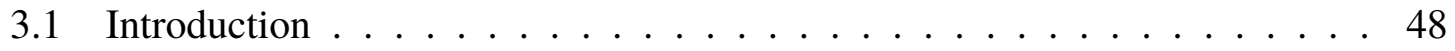

3.2 Experimental Procedure and Results . . . . . . . . . . . . . . . 52

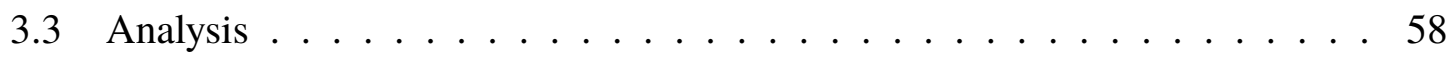

3.4 Electric Field Characterization . . . . . . . . . . . . . . . . 62

3.5 Conclusion . . . . . . . . . . . . . . . . . 69

4 Dipole-Dipole Resonance Line Shapes in a Cold Rydberg Gas 70

4.1 Introduction . . . . . . . . . . . . . 70

4.2 Experimental Procedure $\ldots \ldots \ldots \ldots$. . . . . . . . . . . . . .

4.3 Experimental Results . . . . . . . . . . . . . . . 76

4.4 Analysis and Discussion $\ldots \ldots \ldots$. . . . . . . . . . . 77

4.5 Conclusion . . . . . . . . . . . . . . . . . 88 
5 Towards Controlling Minimum Atom Separation in a Cold Gas 90

5.1 Introduction . . . . . . . . . . . . . . . . 90

5.2 Chirped Frequency Experiment . . . . . . . . . . . . . . . . 97

5.2.1 Experimental Procedure . . . . . . . . . . . . . 97

5.2.2 Results and Analysis . . . . . . . . . . . . . . . . . . 102

5.3 Fixed Frequency Experiment . . . . . . . . . . . . . . 111

5.3.1 Experimental Procedure ................. 111

5.3.2 Results and Analysis . . . . . . . . . . . . . 115

5.4 Model Improvements . . . . . . . . . . . . . . . . . . . . . 119

5.5 Future Steps . . . . . . . . . . . . . . . . 125

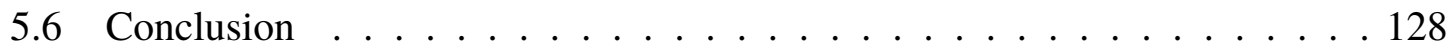

6 Summary and Conclusion $\quad 129$ 


\section{List of Figures}

1.1 A schematic of the energy levels for a pair of atoms under the resonant energy transfer reaction $32 p_{3 / 2} 32 p_{3 / 2} \leftrightarrow 32 s 33 s . \ldots \ldots \ldots \ldots \ldots$

1.2 The calculated energies of the $32 p_{3 / 2} 32 p_{3 / 2}\left(\left|m_{j}\right|=3 / 2\right)$ and $32 s 33 s$ pair states under the Stark effect. Zero energy has been set to the crossing point

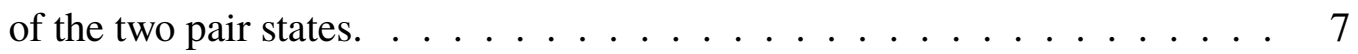

2.1 Schematic drawing of the Vortex Stablewave tunable diode laser [1] . . . 10

2.2 Schematic drawing of the Toptica TA/DL-SHG pro laser. . . . . . . . . 12

2.3 Schematic drawing of the double-pass AOM configuration showing (a) a top-down view of the setup and (b) a side view of the setup. . . . . . . 15 
2.4 Schematic of the Surelite III Nd:YAG laser. The labels H, C, and V represent the horizontal, circular, and vertical polarizations of the light. Not shown here are the SLD and SLT modules that produce the second harmonic 532 $\mathrm{nm}$ light and the third harmonic $355 \mathrm{~nm}$ light, respectively. (a) The voltage across the Pockels cell is $0 \mathrm{~V}$ such that the polarization is only rotated by $\lambda / 4$ after each pass through the $\lambda / 4$ plate and the light is rejected by the polarizer. (b) The voltage across the Pockels cell is $3600 \mathrm{~V}$, creating another $\lambda / 4$ rotation on each pass through the Pockels cell. The net effect of the Pockels cell and waveplate is zero retardation, allowing the light to pass through the polarizer and the cavity to lase [2] . . . . . . . . . 17

2.5 One dimensional energy level schematic of the MOT. The energy of excited state $e$ is split by the Zeeman effect into three sublevels $M_{e}=0, \pm 1$ for $J_{e}=1$. Counterpropagating beams with opposite circular polarization $\sigma_{+}$ and $\sigma_{-}$are given a red-detuning $\delta$. Atoms at $z>0$ are more likely to scatter light from the $\sigma_{-}$beam while atoms at $z<0$ are more likely to scatter light from the $\sigma_{+}$beam. Therefore, the atoms outside of the center of the trap will be pushed towards the center. [3] . . . . . . . . . . . . . 20 
2.6 Schematic representation of the 3D MOT. The magnetic field is provided by a pair of anti-Helmholtz coils represented by the dark rings at the top and bottom of the schematic. An arrow on each shows the direction of the current. The thick black arrows on the axes show the direction of the magnetic field near the center of the trap. The large red arrows are the three pairs of counterpropagating beams with their polarizations labeled $[4,5]$. The large, dashed red arrow is the re-pump beam that is used to prevent pumping to the $5 s_{1 / 2}, F=2$ "dark" ground state as discussed in Section

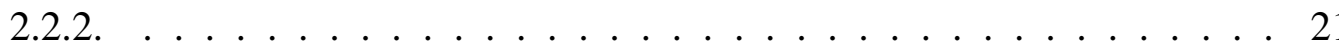

2.7 Schematic diagram of the energy levels of ${ }^{85} \mathrm{Rb}$ showing the trapping and re-pumping transitions along with the hyperfine structure of the $5 s_{1 / 2}$ and $5 p_{3 / 2}$ states (energy levels are not to scale). . . . . . . . . . 26

2.8 The energy levels for a three-level dressed atom. The energy levels on the left side are the energies for states $a, b$, and $c$ in the dressed atom picture without interaction. States $|1\rangle$ and $|2\rangle$ are the states with the atom-light interaction. State $c$ remains unchanged as the laser driving states $a$ and $b$ is far off resonance for state $c$. The energy gap between states $b$ to $c$ is $\omega_{1} \ldots$. . 32

2.9 Spectroscopy of the $32 p_{3 / 2}$ Rydberg state of ${ }^{85} \mathrm{Rb}$. The bold line is primarily the $m_{j}=1 / 2$ state and the thin line is primarily the $m_{j}=3 / 2$ state. Both states show a primary peak and a smaller satellite peak consistent with an Autler-Townes doublet [6]. The small satellite features, left of the main $m_{j}=1 / 2$ peak and right of the main $m_{j}=3 / 2$ peak in, are due to excitation of the $m_{j}=3 / 2$ and $m_{j}=1 / 2$ states, respectively. . . . . . . . . . 33 
2.10 The energy splitting between the $32 p_{3 / 2}\left|m_{j}\right|=3 / 2$ state and the $32 p_{3 / 2}$ $\left|m_{j}\right|=1 / 2$ state. The points are experimental data collected by performing spectroscopy on the $m_{j}$ states. The solid line is a calculation of the difference between the $m_{j}$ states done by calculating the Stark shift for all of the states near to the $32 p$ state. The inset shows the points near the minimum of the splitting [6] . . . . . . . . . . . . . . . . 35

2.11 Schematic of a $1 / r$ potential with (a) no external electric field and (b) an external electric field $F$. The solid lines represent bound energy states of the electron. When the field $F$ is applied, the electron in the uppermost state is above the barrier and can escape from the potential. . . . . . . . . . 37

2.12 Schematic of the micro-channel plate (MCP) detector set up in a chevron configuration for collecting ions. The detector consists of two microchannel plates sandwiched between three thin metal plates. For ion detection, the front plate is held between $-1500 \mathrm{~V}$ to $-2000 \mathrm{~V}$ and the back plate is held slightly above ground while the middle plate is allowed to float. The electrons emitted by the MCPs are collected on the collection plate which is held at $100 \mathrm{~V}$. The voltage on the back plate is measured as a function of time and indicates the relative number of ions hitting the detector versus time $[5] \ldots \ldots \ldots \ldots \ldots$

2.13 A sample trace of the signal from the Fabry-Pérot interferometer. The cavity length of the FPI is set such that the transmission through the cavity is approximately half of the maximum transmission at the frequency we wish to lock. . . . . . . . . . . . . . . . . 40 
2.14 A schematic of the frequency locking system for the Toptica TA-SHG Pro. The red line is the infrared $(960 \mathrm{~nm})$ light going from the master diode laser to the Fabry-Pérot Interferometer (SA200-3B). The signal from the FPI goes to the amplifier (SA201) and from there goes to the feedback controller (PID 110). The control signal goes from the PID 110 to the master diode laser and adjusts the frequency. The piezo driver (TPZ001) sends a voltage to the FPI while the temperature controller (HTC-3000) sends a current to the heaters wrapped around the FPI and reads the temperature of the FPI using a thermistor. . . . . . . . . . . . . . . . . 42

2.15 A schematic drawing of the "T" used for controlling the pressure of the Fabry-Pérot Interferometer. (a) The top view shows the "T" shape with the FPI resting inside the main part of the "T". (b) The side view shows the electrical feedthroughs and the valve that is used for pressure tuning of the FPI frequency. . . . . . . . . . . . . . . . . . . . . 44 
3.1 (a),(c) Probabilities for finding atoms in $26 s+25 p$ (green, fastest decay), $32 s$ (red, intermediate decay), and $40 s$ (blue, slowest decay) as a function of detection time $\tau$ for Rydberg densities of $\rho \sim 3 \times 10^{9} \mathrm{~cm}^{-3}(\mathrm{a}, \mathrm{b})$ and $\rho \sim$ $1.5 \times 10^{9} \mathrm{~cm}^{-3}(\mathrm{c}, \mathrm{d})$. Note that the sum of the $26 \mathrm{~s}$ and $25 p$ populations is shown since their corresponding features could not be adequately separated in the field-ionization signal. Vertical bars show the experimental data with uncertainties, and the solid curves are calculated as described in the text. Measurements and calculations for the $40 \mathrm{~s}$ decay extend to $500 \mu$ s where the remaining population is negligible. (b),(d) Probabilities for finding the atoms in $26 p$ (green, fastest rise and decay), $32 p$ (red, intermediate rise and decay), and $40 p$ (blue, slowest rise and decay) levels as a function of detection time $\tau$. The states are populated by blackbody redistribution from the initial $26 s, 32 s$, and $40 s$ levels, respectively. The data was measured simultaneously with those shown in (a) and (c). Vertical bars show the experimental data with uncertainties and the solid curves are calculated as described in the text. The measured $p$-state probabilities are normalized to the calculations as described in the text. The calculations have no free parameters and consider only the effects of spontaneous emission and blackbody radiation on isolated atoms. . . . . . . . . . . . 55 
3.2 Probabilities for finding atoms in $26 p$ (green, fastest decay), $32 p$ (red, intermediate decay), and $40 p$ (blue, slowest decay) as functions of detection time $\tau$ for Rydberg densities of $\rho \sim 3 \times 10^{9} \mathrm{~cm}^{-3}$ (a) and $\rho \sim 1.5 \times 10^{9}$ $\mathrm{cm}^{-3}$ (b). Vertical bars show the experimental data with uncertainties and the solid curves are calculated as described in the text. Measurements and calculations for the $40 p$ decay extend to $500 \mu$ s where the remaining population is negligible. The calculations have no free parameters and consider only the effects of spontaneous emission and blackbody radiation on isolated atoms. . . . . . . . . . . . . . . . . 56

3.3 Measured $32 p_{3 / 2}\left|m_{j}\right|=1 / 2$ (bold line) and $\left|m_{j}\right|=3 / 2$ (thin line) excitation probabilities as a function of Rydberg laser frequency in zero applied field. The two data curves are obtained simultaneously in the same laser frequency scan. The small feature on the left (right) of the main $\left|m_{j}\right|=1 / 2(3 / 2)$ peak is the result of imperfect discrimination of the $\left|m_{j}\right|=1 / 2$ and $3 / 2$ components via SSFI. The additional peak on the right of the main feature in each trace is due to the trap-laser dressing of the $5 p_{3 / 2}$ and $5 s$ levels. Its frequency shift from the main peak reflects the Autler-Townes splitting of the $5 p_{3 / 2}$ initial state. See Section 2.2.3 for more information on the Autler-Townes splitting. . . . . . . . . . . . . . . . . . 64 
3.4 Difference (i.e. splitting) in the transition energies for exciting $32 p_{3 / 2}$ $\left|m_{j}\right|=1 / 2,3 / 2$ from $5 p_{3 / 2}$ as a function of applied electric field. Filled circles are measurements and the solid curve is the result of a numerical Stark map calculations assuming orthogonal "offset" and "residual" electric field components due to the MCP of $2.758 \mathrm{~V} / \mathrm{cm}$ and $1.38 \mathrm{~V} / \mathrm{cm}$, respectively. The inset shows a magnified view of the portion of the main figure within the dashed window. . . . . . . . . . . . . . . . . 66 
4.1 $32 p_{3 / 2} 32 p_{3 / 2} \rightarrow 32 s 33 s$ DD-resonance line shapes, showing population transfer to $33 \mathrm{~s}$ as a function of applied tuning field at various Rydberg densities: $\rho=2 \times 10^{8}$ (filled circles); $\rho=1 \times 10^{9}$ (filled triangles); and $\rho=3 \times 10^{9} \mathrm{~cm}^{-3}$ (filled squares). These line shapes were measured using a step function rising and falling edge for the tuning pulse and a tuning pulse length of $\tau=15 \mu \mathrm{s}$. The measured signals are not individually normalized, so the relative heights of the profiles reflects the difference in resonant transition probability. The baseline, corresponding to zero population transfer, is the same for the three data sets. The horizontal axis shows the applied tuning field due to the rods. The resonance line centers are shifted from the expected resonance condition, $F_{0}=12.5 \mathrm{~V} / \mathrm{cm}$, due to the presence of the MCP offset field described in Sections 3.4 and 4.3. The three data sets are acquired with the excitation beam focused at (slightly) different locations within the MOT. The relative shifts of the line centers are due to the variation in the offset field within the FWHM of the atom cloud. As described in Section 4.4, the inhomogeneity in the offset field is also responsible for the Gaussian line shapes observed at low Rydberg density. The solid (red) line through the lowest density data is the best Gaussian fit of the inhomogeneously broadened line shape. The solid (blue and green) lines through the higher density data are fits to the cusp line shape expected for a random ensemble, as described in Section 4.4. The small peaks indicated by arrows on either side of the resonance data are sidebands due to oscillations in the tuning pulse and vanish when an exponential rising and falling pattern is applied to the tuning pulse. . . . . . . . . . 78 
4.2 Resonance width as a function of Rydberg density. Measured widths are shown as points with the shapes showing different tuning pulse lengths $\tau$ : $200 \mathrm{~ns}$ (green squares), $1 \mu \mathrm{s}$ (orange cirlces), $15 \mu \mathrm{s}$ (purple triangles). The two dotted lines are the calculated width as described in Section 4.4 (lower) and the calculated width multiplied by a factor of 2 (upper) assuming only nearest-neighbor interactions and $15 \mathrm{MHz}$ of inhomogeneous broadening due the offset field. The points for $\tau=15 \mu$ s include data taken with both a long $3 \mu$ s excitation laser pulse and a short $10 \mathrm{~ns}$ excitation laser pulse. No significant difference in the profile widths for the long and short pulse excitations is expected or observed. . . . . . . . . . . . . . . . 79

4.3 Pair-energy detuning from resonance as a function of applied electric field. The filled circles are experimentally determined values of $\delta$ obtained from Stark shift measurements for the $32 p_{3 / 2}\left|m_{j}\right|=3 / 2,32 s$, and $33 s$ levels as a function of the applied field. At these low fields, the energies of all three levels shift quadratically with the field. The solid line is a quadratic fit to the data. Near resonance, the variation in $\delta$ is approximately linear with a slope of $170 \mathrm{MHz} /(\mathrm{V} / \mathrm{cm})$ (dashed line) . . . . . . . . . . . . . . . 80

4.4 Comparsion of the cusp (solid line) and Lorentzian (dashed line) line shapes expected for ensembles with random [see Eq. 4.5] and uniform [see Eq. 4.3] atom separation, respectively. The Lorentzian profile assumes the most probable value of $R$ at the Rydberg density used to compute the cusp. Note the cusp's broad, large amplitude wings and relatively narrow central peak. . 83 
5.1 Energy of a laser dressed, DD resonant, atom pair with control laser detuning $\Delta=30 \mathrm{MHz}$ and single-atom Rabi frequency (a) $\Omega=0 \mathrm{MHz}$ and (b) $\Omega=19$ MHz. In (a), $W_{1, N+2}$ is the energy of the $5 p_{3 / 2} 5 p_{3 / 2}$ trap state pair plus two blue-detuned photons. $W_{2, N+1}$ is the energy of the singly-excited Rydberg state, $5 p_{3 / 2} 32 p_{3 / 2}$ or $32 p_{3 / 2} 5 p_{3 / 2}$ plus one blue-detuned photon. $W_{3, N}$ and $W_{4, N}$ are the doubly-excited Rydberg states, $32 p_{3 / 2} 32 p_{3 / 2}+32 s 33 s$ and $32 p_{3 / 2} 32 p_{3 / 2}-32 s 33 s$, respectively, with no additional photons. The energy $W_{1, N+2}$ is defined as 0 . The dashed horizontal line is the energy of the doubly-excited Rydberg state for atoms with infinite separation. In (b), the energies have developed laser-induced avoided crossings where the doublyexcited Rydberg state energy with dipole-dipole interaction crosses with the other energy levels. The remaining flat energy level is the $5 p_{3 / 2} 32 p_{3 / 2}-$ $32 p_{3 / 2} 5 p_{3 / 2}$ "dark" state that does not couple to the rest of the system. See Figure 5.2 for the composition of the states with laser interaction shown in

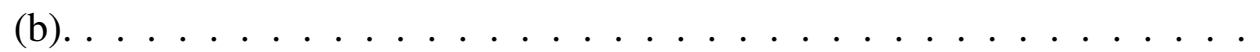

5.2 Energy of a laser dressed, DD resonant, atom pair for control laser detuning $\Delta=30 \mathrm{MHz}$ and Rabi frequency $\Omega=19 \mathrm{MHz}$, which allows for an adiabatic transition. The dashed lines are the energy levels as shown in Figure 5.1(b). The colors in each panel show the probability for the atom pairs to be in state (a) $5 p 5 p$, (b) $5 p 32 p+32 p 5 p$, (c) $32 p 32 p+32 s 33 s$, and (d) $32 p 32 p-32 s 33 s$. The $5 p 32 p-32 p 5 p$ state probability is not shown as it does not interact with the other states and resides entirely in the straight, horizontal dashed line. . . . . . . . . . . . . . . . . . . . . . 94 
5.3 A sample field ionization trace for the fixed frequency control laser described in Section 5.3.1 with detuning $\Delta=15 \mathrm{MHz}$, MOT formation time $t_{\text {on }}=0.25 \mathrm{~s}$, and an electric field of $F=18.6 \mathrm{~V} / \mathrm{cm}$. The vertical lines on the right show the gate for the Rydberg states and the vertical lines on the left show the gate for the additional ions. The time $\mathrm{t}=0$ is set to the arrival of the field ionization trigger. . . . . . . . . . . . . . 100

5.4 (a) Total Rydberg signal, (b) ion signal, and (c) MOT fluorescence as a function of initial detuning $\Delta_{I}$. The target detuning $\Delta_{T}=30 \mathrm{MHz}$ in all cases. The filled circles are for step sizes of $1 \mathrm{MHz}$ and the filled triangles are for step sizes of $5 \mathrm{MHz}$. The green points are for an applied electric field of $12.6 \mathrm{~V} / \mathrm{cm}$ (on resonance) and the orange points are for an applied electric field of $18.6 \mathrm{~V} / \mathrm{cm}$ (off resonance). Detection of the Rydberg atoms and ions was done $2 \mathrm{~ms}$ after the end of the frequency sweep (with the control laser still on). The Rydberg and ion signals are both normalized by the MOT fluorescence. No frequency chirp occurs for $\Delta_{I}=30 \mathrm{MHz}$. . . . 101

5.5 The total Rydberg signal as a function of Rydberg excitation laser power at a fixed excitation laser detuning of $\Delta=30 \mathrm{MHz}$. A $2 \mu \mathrm{s}$ AOM chopped pulse was used for the excitation.The green points were taken in a total electric field of $12.6 \mathrm{~V} / \mathrm{cm}$ and the orange points were taken in a total field of $18.6 \mathrm{~V} / \mathrm{cm}$. The lines are linear fits with their $y$-intercept set to 0 . The Rydberg signal is normalized by the MOT fluorescence. The ratio between the two slopes $m_{18.6} / m_{12.6} \sim 2 \ldots \ldots$. . . . . . . . . . . 103 
5.6 Total Rydberg signal as a function of Rydberg excitation laser pulse length for detuning $\Delta=$ (a) $20 \mathrm{MHz}$, (b) $30 \mathrm{MHz}$, and (c) $40 \mathrm{MHz}$. The different solid lines are for excitation at electric fields of $12.6 \mathrm{~V} / \mathrm{cm}$ (green), 14.7 V/cm (orange), 18.6 V/cm (purple), and 22.6 V/cm (pink). See Figure 5.7 for total ion signals from the same measurement. The Rydberg signals have been normalized by the MOT fluorescence. . . . . . . . . . . . . 105

5.7 Total ion signal as a function of Rydberg excitation laser pulse length for detuning $\Delta=$ (a) $20 \mathrm{MHz}$, (b) $30 \mathrm{MHz}$, and (c) $40 \mathrm{MHz}$. The different solid lines are for excitation at electric fields of $12.6 \mathrm{~V} / \mathrm{cm}$ (green), $14.7 \mathrm{~V} / \mathrm{cm}$ (orange), $18.6 \mathrm{~V} / \mathrm{cm}$ (purple), and 22.6 V/cm (pink). See Figure 5.6 for total Rydberg signals from the same measurement. The ion signals have been normalized by the MOT fluorescence. . . . . . . . . . . . . 106

5.8 Rydberg signal as a function of detuning from the zero-field $32 p_{3 / 2}$ excitation frequency with an applied electric field of (a) $12.4 \mathrm{~V} / \mathrm{cm}$, (b) 12.6 $\mathrm{V} / \mathrm{cm}$, and (c) $13.0 \mathrm{~V} / \mathrm{cm}$. The large peak seen at lower frequencies is the $32 p_{3 / 2}\left|m_{j}\right|=3 / 2$ Rydberg state. The small peak seen at higher frequencies is a satellite peak identified as transitions to the $32 p_{3 / 2}\left|m_{j}\right|=1 / 2$ Rydberg state from the lower-lying hyperfine levels of the $5 p_{3 / 2}$ trap state. . . . . . 108 
5.9 Detuning from the zero electric field Rydberg excitation laser frequency as a function of applied electric field. The points are the measured excitation frequencies for the large peak (filled circles) and small peak (filled triangles) from Figure 5.8. The lines are calculated excitation frequencies based on a Stark map calculation with the solid lines being excitation to the $32 p_{3 / 2}$ $\left|m_{j}\right|=1 / 2$ state and dashed lines being excitation to the $32 p_{3 / 2}\left|m_{j}\right|=3 / 2$ state. The colors are for excitation from the $5 p_{3 / 2}$ trap state with hyper-fine state $F=4$ (black), 3 (blue), 2 (red), and 1 (green). The measured points have been shifted by $-0.5 \mathrm{~V} / \mathrm{cm}$ to better align with the calculation. . . . . . 110

5.10 Nearest-neighbor probability distribution for a nominal density of $6 \times 10^{9}$ $\mathrm{cm}^{-3}$. The shaded sections are for separations of less than $1 \mu \mathrm{m}$ (furthest left and shaded red), $1.7 \mu \mathrm{m}$ (center and shaded blue), and $2.5 \mu \mathrm{m}$ (furthest rigth and shaded green). $2.7 \%$ of the atoms are in the red section, $12 \%$ are in the red and blue sections, and $33 \%$ are in all of the shaded sections. . . . 112

5.11 Rydberg atom population as a function of re-pump turn-off time $t_{\text {off }}$. Complete dissipation of the MOT is achieved after approximately $1 \mathrm{~s}$. . . . . . 114

5.12 (a) Normalized Rydberg population and (b) normalized ion population for a control laser detuning $\Delta=15 \mathrm{MHz}$. Measurements were made at electric fields of $F=12.6 \mathrm{~V} / \mathrm{cm}$ (green points) and $18.6 \mathrm{~V} / \mathrm{cm}$ (orange points). Both Rydberg and ion populations were normalized by dividing by the total ion yield (sum of the Rydberg and ion populations) . . . . . . . . . . . 116 
5.13 (a) Normalized Rydberg population and (b) normalized ion population for a control laser detuning $\Delta=30 \mathrm{MHz}$. Measurements were made at electric fields of $F=12.6 \mathrm{~V} / \mathrm{cm}$ (green points) and $18.6 \mathrm{~V} / \mathrm{cm}$ (orange points). Both Rydberg and ion populations were normalized by dividing by the total ion yield (sum of the Rydberg and ion populations) . . . . . . . . . . . 117

5.14 (a) Normalized Rydberg population and (b) normalized ion population for a control laser detuning $\Delta=40 \mathrm{MHz}$. Measurements were made at electric fields of $F=12.6 \mathrm{~V} / \mathrm{cm}$ (green points) and $18.6 \mathrm{~V} / \mathrm{cm}$ (orange points). Both Rydberg and ion populations were normalized by dividing by the total ion yield (sum of the Rydberg and ion populations) . . . . . . . . . . . 118

5.15 Adiabatic crossing probability as a function of Rabi Frequency $\Omega$ for control laser detunings $\Delta$ of (a) $10 \mathrm{MHz}$, (b) $15 \mathrm{MHz}$, (c) $20 \mathrm{MHz}$, (d) $30 \mathrm{MHz}$, and (e) $40 \mathrm{MHz}$. The adiabatic crossing probability is given in Equation 5.1. The dotted vertical line is a reference for the control laser Rabi frequency used in the experiments, approximately 4 MHz. . . . . . . . . . . . . . . 120 
5.16 Energy of the laser dressed, DD resonant, atom pair for a model including the $5 s$ ground state. The calculation was done using a trap laser detuning $\Delta_{1}=20 \mathrm{MHz}$ and single-atom Rabi frequency $\Omega_{1}=16 \mathrm{MHz}$ and a control laser detuning $\Delta_{2}=30 \mathrm{MHz}$ and single-atom Rabi frequency $\Omega_{2}=22$ MHz. The energy levels are shown in the solid lines in (a). (b) - (f) shows the energy levels in the dashed lines with the color giving the probability for the atom pairs to be in the state (b) $5 s 5 s$, (c) $5 s 5 p+5 p 5 s$, (d) $5 p 5 p$, (e) $5 p 32 p+32 p 5 p$, (f) $32 p 32 p+32 s 33 s$. The repelling Rydberg-Rydberg state $32 p 32 p-32 s 33 s$, along with the non-interacting, "dark", states $5 s 5 p-5 p 5 s$ and $5 p 32 p-32 p 5 p$ are not shown. . . . . . . . . . . . . 122

5.17 Rough model showing the laser dressed, DD resonant, atom pair energies and including the $5 s$ ground state and the natural energy width of the $5 p_{3 / 2}$ state. The calculation was done using a trap laser detuning $\Delta_{1}=20 \mathrm{MHz}$ and single-atom Rabi frequency $\Omega_{1}=16 \mathrm{MHz}$ and a control laser detuning $\Delta_{2}=30 \mathrm{MHz}$ and single-atom Rabi frequency $\Omega_{2}=22 \mathrm{MHz}$. The bold black lines are the average energies for each state. The gray bars around each line are the energy widths of each state assuming the natural line width

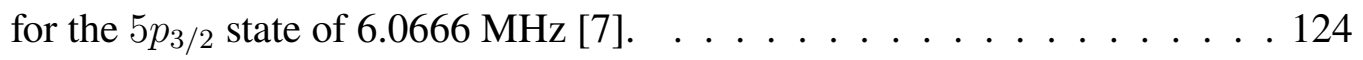




\section{Chapter 1}

\section{Introduction}

\subsection{Motivation}

Atom-atom interactions in Rydberg systems, particularly long-range dipole-dipole interactions, have been a topic of intense study over the last few decades. Beginning with experiments examining collisions between thermal atoms [8], the development of laser cooling and trapping has allowed these experiments to move to a regime where the atoms are nearly motionless over the course of an experiment [9-39]. This is the, so-called, nearlyfrozen or frozen gas regime. Rydberg atoms in the frozen gas regime allow us to explore long-range dipole-dipole interactions and exploit them to study few- to many-body quantum physics. Rydberg atoms are an ideal medium for this study as they are highly sensitive to external electric fields [8], allowing for strong, long-range interactions. Additionally, dipole-dipole coupled Rydberg atoms are a potential platform for quantum information applications [12, 40-43]. 
This dissertation describes experiments investigating dipole-dipole interactions in a cold Rubidium Rydberg gas. We examined how dipole-dipole interactions work to shut off collective decay effects (Chapter 3), how many-body effects and atomic motion influence our ability to characterize and control dipole-dipole interactions (Chapter 4), and how dipole-dipole interactions might be used to control the motion of atoms (Chapter 5). This work builds upon the research from many other groups [9-11,22,44]. It also follows directly from previous work by Mary Kutteruf and Tao Zhou, former students in our group. The work of Mary Kutteruf focused on the coherence of dipole-dipole interactions [5]. The work of Tao Zhou focused on the evolution of Rydberg wave packets and the transfer of wave packet coherence due to dipole-dipole interactions [45], in addition to the collective decay investigation reproduced here (Chapter 3). My experiments approach the study of dipoledipole interactions by examining the strength of the interaction itself, its characterization in the presence of atom motion and stray fields, and whether that interaction might be used to physically manipulate relative atom positions within a cold gas.

\subsection{Atomic Units}

Throughout this dissertation we make use of atomic units, a unit system commonly used in atomic physics $[46,47]$. Atomic units are defined with

$$
\hbar=m_{e}=e=\frac{1}{4 \pi \epsilon_{0}}=1
$$

where $\hbar$ is the reduced Planck's constant $(h / 2 \pi), m_{e}$ is the mass of the electron, $-e$ is the fundamental charge of the electron, and $\epsilon_{0}$ is the permittivity of free space. Conversions 


\begin{tabular}{|c|c|c|}
\hline Atomic Unit & Definition & SI Value \\
\hline Charge & $e$ & $1.602188 \times 10^{-19} \mathrm{C}$ \\
\hline Mass & $m_{e}$ & $9.10939 \times 10^{-31} \mathrm{~kg}$ \\
\hline Angular momentum & $\hbar$ & $1.05457 \times 10^{-34} \mathrm{~J} /(\mathrm{s} \mathrm{rad})$ \\
\hline Energy & $m_{e} e^{4} / \hbar^{2}$ & $4.35975 \times 10^{-18} \mathrm{~J}$ \\
\hline Length & $\hbar^{2} / m_{e} e^{2}$ & $5.29177 \times 10^{-11} \mathrm{~m}$ \\
\hline Time & $\hbar^{3} / m_{e} e^{4}$ & $2.41888 \times 10^{-17} \mathrm{~s}$ \\
\hline Electric dipole moment & $\hbar^{2} / m_{e} e$ & $8.47836 \times 10^{-30} \mathrm{C} \mathrm{m}$ \\
\hline Magnetic dipole moment & $e \hbar / 2 m_{e}$ & $9.27402 \times 10^{-24} \mathrm{~J} / \mathrm{T}$ \\
\hline
\end{tabular}

Table 1.1: Definition and conversion factors for typical atomic units $[47,48]$.

from atomic units to SI units are given in Table 1.1.

\subsection{Rydberg Atoms}

All of the experiments in the following chapters involve exciting ${ }^{85} \mathrm{Rb}$ atoms to Rydberg states. A Rydberg atom is an atom where an electron bound to the atom has been excited to a high principal quantum number $n$ (typically $n \gtrsim 10$ ) [8]. Rydberg atoms have a number of useful properties that scale with various powers of $n$ (see Table 1.2). Among the properties of interest to us are the large orbital radius of the electron $\left(n^{2}\right)$, the long radiative lifetime $\left(n^{3}\right)$, and large dipole-dipole interactions $\left(n^{4}\right)$. The large transition dipole moment $\left(n^{2}\right)$ and polarizability $\left(n^{7}\right)$ also show that Rydberg atoms are highly susceptible to external electric fields.

For non-hydrogenic Rydberg electrons with low orbital angular momentum $\ell$, we must also consider perturbations caused by the ion core. In a Rydberg atom, the excited electron spends the majority of its time far from the ion core where it is sensitive only to the net charge of the core. When the electron approaches the core, it becomes sensitive to the distribution 


\begin{tabular}{|c|c|}
\hline Property & $n$ dependence \\
\hline Binding energy & $n^{-2}$ \\
\hline Energy between adjacent $n$ states & $n^{-3}$ \\
\hline Orbital radius & $n^{2}$ \\
\hline Geometric cross section & $n^{4}$ \\
\hline Dipole moment & $n^{2}$ \\
\hline Polarizability & $n^{7}$ \\
\hline Radiative lifetime & $n^{3}$ \\
\hline Fine-structure interval & $n^{-3}$ \\
\hline
\end{tabular}

Table 1.2: Properties of Rydberg atoms [8].

of charge within the core, leading to modifications in the energy and wavefunction of the Rydberg state relative to hydrogen. The energy change can be modeled as

$$
W=-\frac{R y}{\left(n-\delta_{\ell}\right)^{2}}=-\frac{R y}{n^{* 2}}
$$

where $R y=k^{2} Z^{2} e^{4} m_{e} / 2 \hbar^{2}=1 / 2$ is the Rydberg constant (assuming infinite nuclear mass) in atomic units, $n$ is the principal quantum number, $\delta_{\ell}$ is the quantum defect for orbital angular momentum number $\ell$, and $n^{*}$ is the effective principal quantum number [8]. In ${ }^{85} \mathrm{Rb}$, the quantum defect $\delta_{\ell}$ has typical values $\delta_{32 p}=2.642016$ and $\delta_{32 s}=3.131394$ and is typically larger at low $\ell$ and approaches 0 at higher $\ell$ [8].

\subsection{Dipole-Dipole Interactions and Resonant Energy Trans- fer}

All of the experiments described below investigate strong, long-range, dipole-dipole interactions between Rydberg atoms. Dipole-dipole interactions are mutual interactions 
between pairs of atoms. For example, let us assume we have a pair of atoms with the first atom in state $|n S\rangle$ and the second atom in state $|n P\rangle$. This atom pair is coupled by an exchange interaction $n S+n P \leftrightarrow n P+n S$ of the form

$$
V_{D D} \propto \frac{|\langle n S|r| n P\rangle|^{2}}{R^{3}}
$$

where $\langle n S|r| n P\rangle$ is the dipole transition matrix element and $R$ is the separation between the atoms. The strength of the interaction comes from the $n^{2}$ scaling of $\langle n S|r| n P\rangle$ for states with approximately the same principle quantum number $n$. Since $V_{D D}$ scales as $n^{4}$ for large $n$, we get incredibly strong interactions, even at large separations [8].

Resonant energy transfer, also known as Förster resonant energy transfer, is a process in which one atom in a pair gains electronic energy at the expense of the other [20,29,40,49]. The process is considered resonant if the electronic energy gain on one atom precisely matches the loss on the other, i.e., no center of mass energy is transferred. Resonant energy transfer can allow for the exchange of energy over large distances as it is often mediated by the dipole-dipole interaction. For example, let us use the interaction studied in Chapters 4 and 5 in which a pair of atoms initially in the $32 p_{3 / 2}\left|m_{j}\right|=3 / 2$ Rydberg state undergo a Stark-tuned resonant energy transfer reaction, nominally $32 p_{3 / 2} 32 p_{3 / 2} \leftrightarrow 32 s 33 s$, with interaction strength

$$
V_{D D} \propto \frac{\langle 32 p|r| 32 s\rangle\langle 32 p|r| 33 s\rangle}{R^{3}} .
$$

In this resonant energy transfer interaction, one of the $32 p_{3 / 2}$ electrons loses energy and is transferred to the $32 s$ state. The other $32 p_{3 / 2}$ electron gains that energy, making a transition to the $33 \mathrm{~s}$ state (see Figure 1.1). This reaction is most efficient at resonance, 


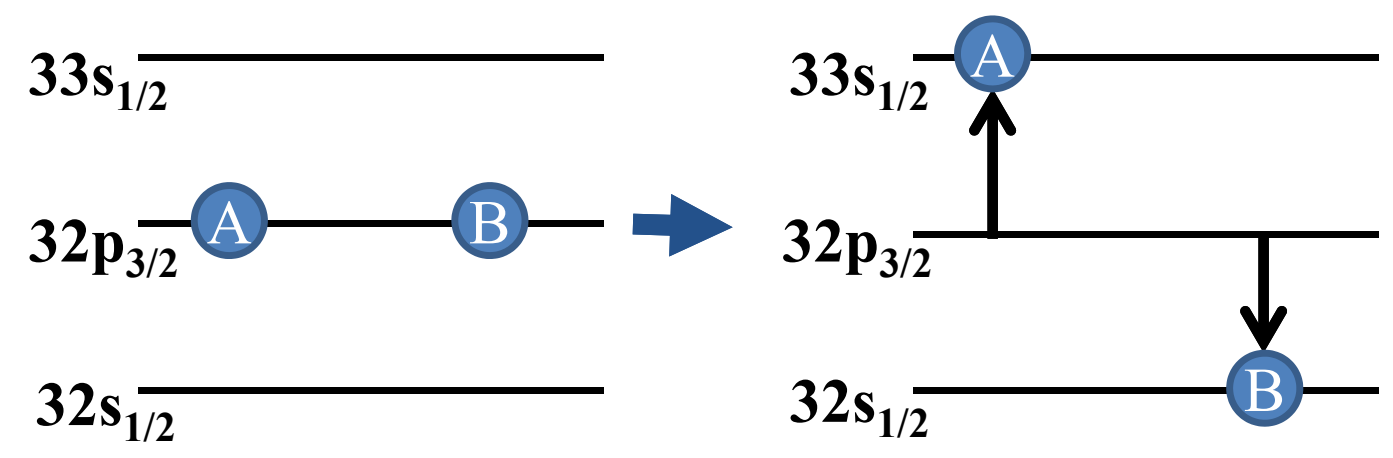

Figure 1.1: A schematic of the energy levels for a pair of atoms under the resonant energy transfer reaction $32 p_{3 / 2} 32 p_{3 / 2} \leftrightarrow 32 s 33 s$.

when the electronic energy of the pair of atoms is the same before and after the reaction, i.e., $2 E_{32 p}-\left(E_{32 s}+E_{33 s}\right)=0$. This resonance situation is particularly easy to achieve in Rydberg atoms due to their high sensitivity to external electric fields. The Stark effect can be used to tune the energy of the Rydberg states to create a system where the initial pair state and final pair state energies are degenerate. Figure 1.2 shows the energies of the $32 p_{3 / 2} 32 p_{3 / 2}\left(\left|m_{j}\right|=3 / 2\right)$ and $32 s 33 s$ pair states as a function of electric field, with the two states at the same energy at the crossing point of $F=11.5 \mathrm{~V} / \mathrm{cm} \mathrm{[20].} \mathrm{In}{ }^{85} \mathrm{Rb}$, reaching degenerate energies in pair states requires low strength electric fields $(F<20 \mathrm{~V} / \mathrm{cm})$ for $p p \leftrightarrow s s^{\prime}$ type reactions with $30<n<40$.

\subsection{Dissertation Structure}

The following chapters describe experiments to study dipole-dipole interactions in a cold Rydberg gas. In Chapter 2, we describe the apparatus used in the experiment including a description of the lasers, vacuum chamber, and various electronics used. It also describes 


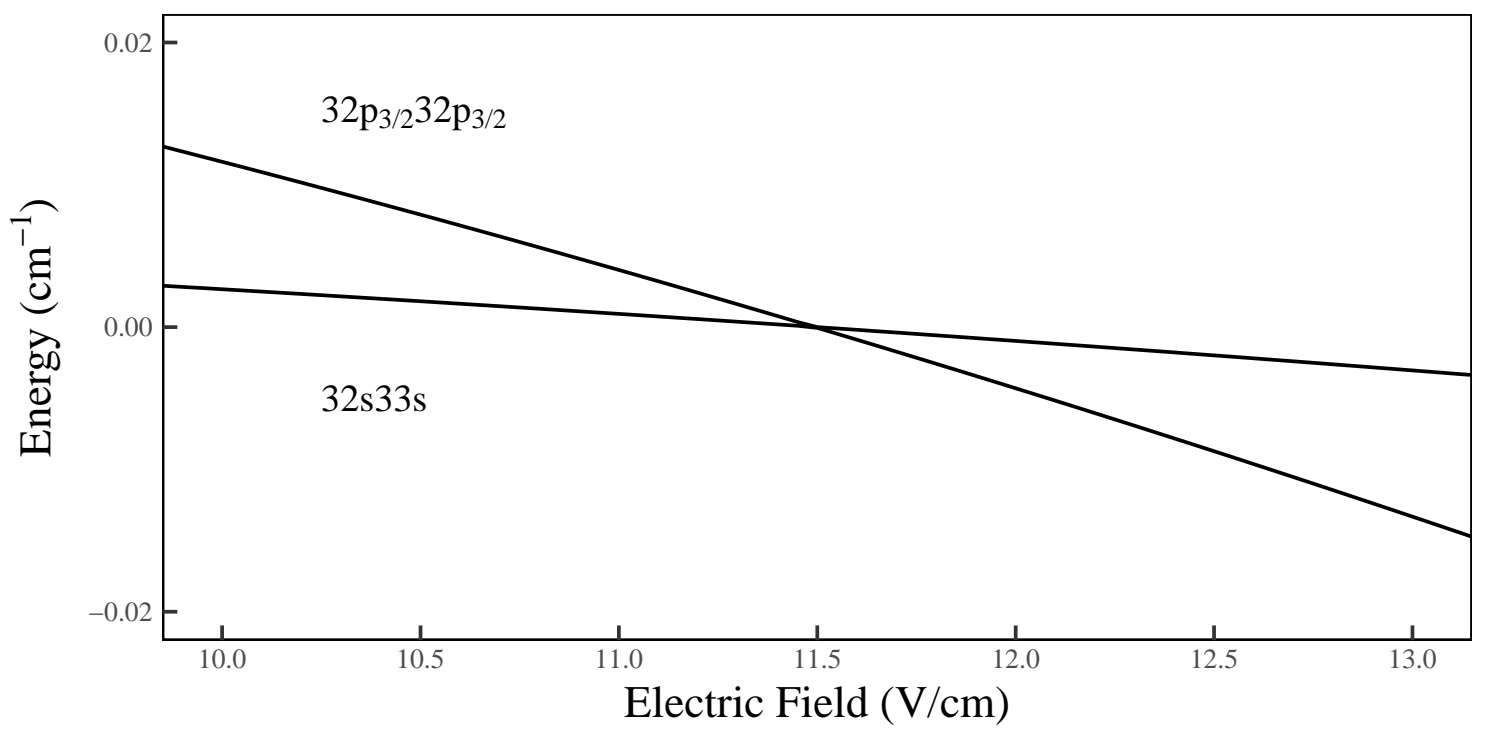

Figure 1.2: The calculated energies of the $32 p_{3 / 2} 32 p_{3 / 2}\left(\left|m_{j}\right|=3 / 2\right)$ and $32 s 33 s$ pair states under the Stark effect. Zero energy has been set to the crossing point of the two pair states.

and provides an introduction to laser cooling and trapping and the magneto-optical trap, along with our implementation and characterization of the trap. Chapter 3 describes an investigation of collective decay effects in cold Rydberg atoms and how dipole-dipole interactions may prevent collective decay. This chapter includes an expanded discussion of our efforts to characterize the electric field within the magneto-optical trap which was key to the interpretation of the results. In Chapter 4, we describe measurements of the line shape of electric-field tuned, dipole-dipole mediated, resonant energy transfer reactions. The roles of beyond 2-body interactions, stray fields, and atom motion are discussed as sources of line shape broadening. Chapter 5 describes an initial exploration of a method for exploiting coherent atom interactions to control the minimum separation of atoms within a cold gas. Finally, in Chapter 6 we present a brief conclusion along with some thoughts on possible future directions and extensions of this work. 


\section{Chapter 2}

\section{Experimental Setup and Equipment}

The experiments described in this dissertation involve the excitation, interaction, and detection of ${ }^{85} \mathrm{Rb}$ atoms excited to Rydberg states in a magneto-optical trap (MOT). These experiments were performed in Room 166/168 of the Physics Building at the University of Virginia. This chapter describes the lasers used for excitation, the equipment used to create the MOT, the electronics used to manipulate and detect the Rydberg atoms, and the software used in detection and analysis.

\subsection{Lasers}

\subsubsection{Diode Lasers}

The creation of our cold atom trap (the MOT, see Section 2.2) is driven by two Vortex Stablewave tunable diode lasers from New Focus. The Vortex Stablewave tunable diode laser is an external cavity diode laser (ECDL) that provides mode-hop free frequency tuning 
and a sub-500 kHz line width. These lasers provide a typical $30 \mathrm{~mW}$ of power and can provide upwards of $50 \mathrm{~mW}$ of power as needed. ECDL's work by using a diffraction grating at grazing incidence to select a narrow lasing wavelength. The light from a diode laser is first collimated using a lens and then directed to the diffraction grating. The first order diffraction from the grating is sent to a prism and reflected as feedback to the diode laser. The zeroth order diffraction from the grating couples the laser light out of the cavity. The prism can be rotated towards or away from the diffraction grating to tune the laser to specific wavelengths (see Figure 2.1). This basic design was first proposed by Littman and Metcalf for use in dye lasers [50] and has since been extended for use in many other systems including diode lasers [51].

"Mode-hop" free tuning of the ECDL is achieved by optimizing two parameters, the prism-grating angle and the cavity length. The cavity length and prism-grating angle are related through

$$
\begin{gathered}
L=\frac{N \lambda_{N}}{2} \\
\lambda=\Lambda\left(\sin \theta_{i}+\sin \theta_{d}\right)
\end{gathered}
$$

where $\mathrm{L}$ is the cavity length, $\lambda_{N}$ is the discrete set of possible modes that can lase, $\Lambda$ is the groove spacing of the diffraction grating, and $\theta_{i}$ and $\theta_{d}$ are the angle of incidence and diffraction, respectively [1]. By appropriately selecting the pivot point of the prism, these two parameters can be synchronized to allow for continuous tuning of the frequency of the diode laser free of any mode-hops, i.e. changes in $N$ [52]. Coarse tuning of the laser wavelength is accomplished by loosening 3 locking screws within the Stablewave housing and rotating the prism mount. The prism and wavelength are fine-tuned by applying a 


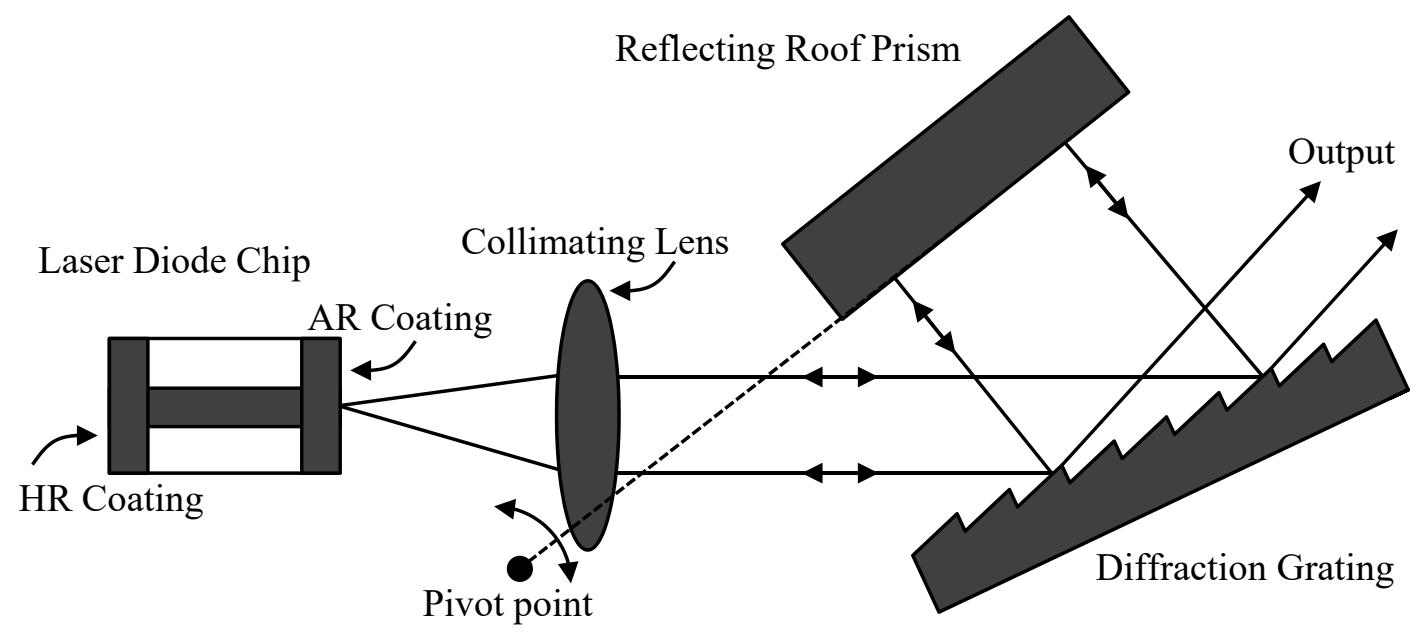

Figure 2.1: Schematic drawing of the Vortex Stablewave tunable diode laser [1].

voltage to a piezoelectric attached to the prism. The piezoelectric can accept voltages from $0 \mathrm{~V}$ to $117 \mathrm{~V}$, but stable operation occurs for voltages in the range of $50 \mathrm{~V}$ to $90 \mathrm{~V}$. Frequency modulation, using an external voltage source for the piezoelectric, is limited by a bandwidth of $3.5 \mathrm{kHz}$. The frequency of the laser can also be fine-tuned by changing the current through, or temperature of, the diode.

Our ${ }^{85} \mathrm{Rb}$ atoms are excited to Rydberg states using a Toptica TA/DL-SHG pro diode laser. The TA-SHG pro is a tunable diode laser that provides $480 \mathrm{~nm}$ light by doubling the $960 \mathrm{~nm}$ light from a master diode laser. The master diode laser (Toptica DL pro) is an ECDL in a modified Littrow-Hänsch configuration. Unlike the Vortex Stablewave diode laser, the DL pro focuses the light from a diode laser onto a reflection grating and then takes the 1st order diffraction and reflects that directly back towards the laser diode. This establishes an optical cavity between the rear facet of the diode and the grating, achieving a bandwidth of 
less than $1 \mathrm{MHz}$. Mode-hop free tuning is achieved using a feed-forward current control system. As the piezoelectric that controls the external resonator length and grating profile is changed, the current is adjusted leading to a change in the internal resonator length [53]. When properly synchronized, this allows for mode-hop free tuning for a typical range of about $20 \mathrm{GHz}$.

As shown in Figure 2.2, the light from the master diode laser is amplified in a tapered amplifier (TA). The TA provides high gain in a single pass cavity where non-seeded emissions are kept negligible by anti-reflection coatings on the interior of both the input and output ports [54]. The TA provides approximately $1 \mathrm{~W}$ of output for approximately $40 \mathrm{~mW}$ of input light. After the TA, the light is sent to a bow-tie-ring second harmonic generation (SHG) cavity. The SHG cavity has a non-linear crystal inside that doubles the frequency of the circulating $960 \mathrm{~nm}$ light. The SHG cavity length is stabilized using the Pound-DreverHall technique $[55,56]$. The cavity length is modulated, creating sidebands on the $960 \mathrm{~nm}$ light that is reflected out of the cavity and into a photodiode. The reflected light measured by the photodiode, after some electronic processing, provides an error signal that varies linearly with the cavity length and passes through zero when the cavity length matches the resonance condition for the laser frequency [54]. This error signal is then sent to a PID feedback system, similar to the ones described in Section 2.3.5, and is used to stabilize the cavity length.

The Toptica TA/DL-SHG pro provides $\sim 160 \mathrm{~mW}$ of laser light at $482 \mathrm{~nm}$. While the nominal frequency bandwidth should be less than $1 \mathrm{MHz}$, we have estimated a maximum bandwidth of approximately $3.9 \mathrm{MHz}$. This was measured by performing spectroscopy on the $32 p_{3 / 2}$ Rydberg state and measuring the full-width at half-maximum (FWHM) of 


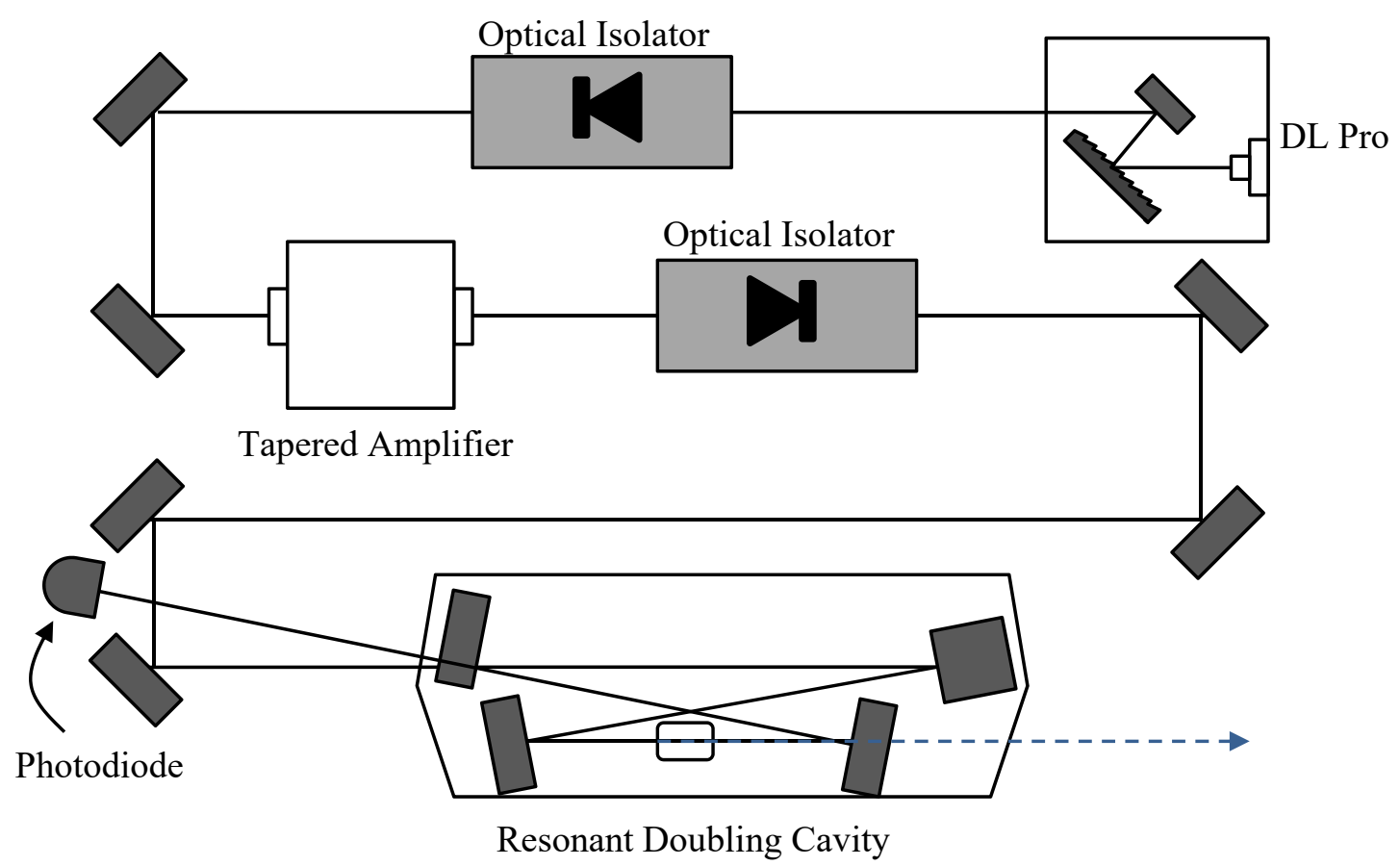

Figure 2.2: Schematic drawing of the Toptica TA/DL-SHG pro laser. 
the resonance. Broadening due to the Doppler effect is negligible in these measurements due to the slow speed of the atoms within the MOT (see Section 2.2). The maximum estimate for the laser bandwidth was extracted from the FWHM of the resonance, which includes contributions from the lifetime of the $5 p_{3 / 2}$ intermediate state, magnetic field inhomogeneities, and electric field inhomogeneities (see Chapter 3 for more details on this measurement).

For the experiments detailed in Chapters 3 and 4, pulsed operation of the Toptica laser was achieved using two different techniques. First, a long pulse $(\sim 3 \mu \mathrm{s})$ was created using an acousto-optic modulator (AOM) by IntraAction Corp (Model AFM-802A1). The AOM uses RF acoustic waves in an optically transparent medium (flint glass) to create a periodic change in the refractive index of the medium. This acts as a diffraction grating with controllable features. Laser light that enters the AOM at the Bragg angle $\theta_{B}=\frac{\lambda F}{2 V}$, where $\lambda$ is the laser wavelength, $F$ is the acoustic frequency, and $V$ is the acoustic velocity of the interaction medium, is diffracted by an angle $\theta_{R}=2 \theta_{B}$ and gives a beam that can be turned on and off by applying a pulse to the AOM driver [57]. We used the first-order diffraction to chop the light from the Toptica laser and create our long pulse. The diffraction efficiency is approximately $60 \%$ due to the relatively large spot size of the beam entering the AOM.

Second, we create a short, $\sim 10 \mathrm{~ns}$, pulse by placing a Pockels cell between crossed polarizers [58]. Our implementation uses the well defined polarization of the Toptica laser and a polarizer after the Pockels cell. When no voltage is applied to the Pockels cell, the polarization of the light is unchanged, and a polarizer dumps the light into a beam block. The index ellipsoid of the Pockels cell is modified by an applied electric field such that the cell acts as a $\lambda / 2$ plate while the field is applied. The electric field is produced by applying 
a voltage across either end of the Pockels cell. While the voltage is applied, the polarization axis of the laser undergoes a $\pi / 2$ rotation allowing it to pass through the polarizer. $\mathrm{A} \sim 3$ $\mathrm{kV}$, fast rising $(<3 \mathrm{~ns})$ pulse is produced by a FastPulse Technology Inc. Lasermetrics 5056 Q-Switch Driver [59]. The $\sim 10 \mathrm{~ns}$ pulse is created by sending the fast-rising pulse first to the front of the Pockels cell and then sending the same fast-rising pulse to the back of the Pockels cell $\sim 10 \mathrm{~ns}$ later, creating a potential difference across the Pockels cell of $\sim 3 \mathrm{kV}$ for only $\sim 10 \mathrm{~ns}$.

Additionally, we use an AOM in a double-pass configuration to achieve precise frequency tuning control of the Toptica laser described above. As the acoustic frequency $F$ of the AOM changes, there is a related change in the deflection of the first-order beam $\Delta \theta_{R}=\frac{\lambda \Delta F}{V}$. The double-pass configuration removes this angular dependence, allowing us to maintain the position of the laser beam while shifting the laser frequency. This configuration is used in the experiments detailed in Chapter 5. The version of the double-pass AOM that we are using is a modification of that presented by Donley et al [60]. As stated above, laser light enters the AOM at the Bragg angle and is diffracted. In addition to being deflected, the first-order diffraction beam is also shifted in frequency by an amount equal to the frequency of the RF driving field applied to the AOM. The frequency of the first-order diffracted beam is $\omega_{1}=\omega_{0} \pm \Omega$ for an incidence angle of $\pm \theta_{B}$, where $\omega_{0}$ is the initial laser frequency and $\Omega$ is the frequency of the RF driving field. After making a first pass through the AOM, the zeroth- and first-order beams enter a cat's eye retroreflector that consists of a plano-convex lens with focal length $f=100 \mathrm{~mm}$ and two flat mirrors at $90^{\circ}$ to one another. These mirrors reflect the light back towards the lens after adding a small vertical translation to the beam. The lens is positioned a distance $f$ from the AOM and the mirrors are a distance $f$ 


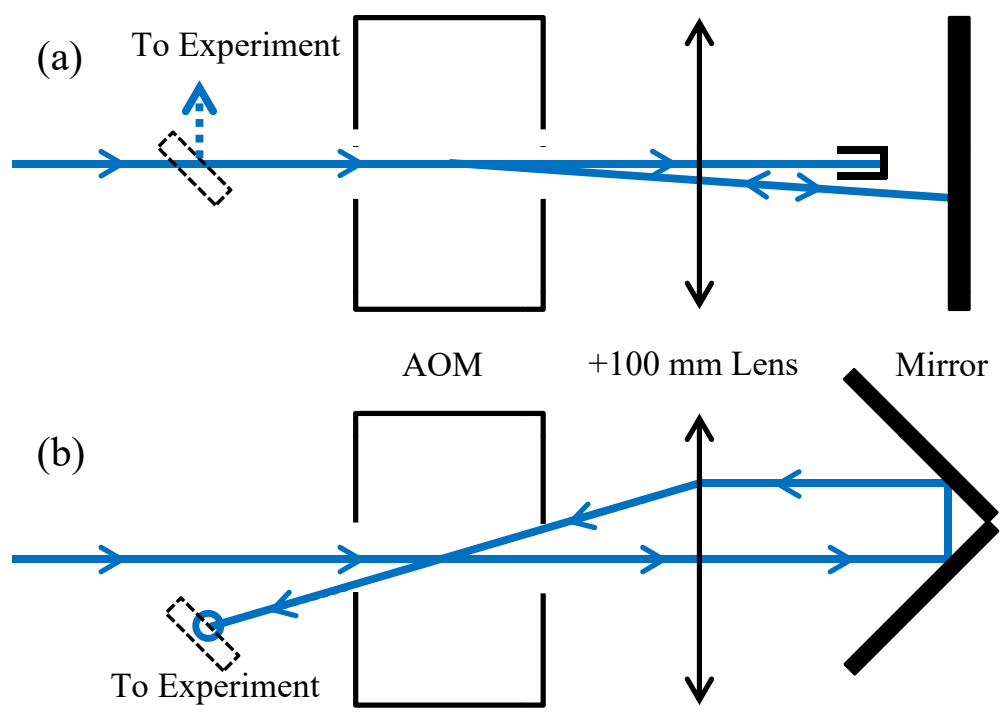

Figure 2.3: Schematic drawing of the double-pass AOM configuration showing (a) a topdown view of the setup and (b) a side view of the setup.

from the lens. The reflected beam returns to the AOM at the same horizontal angle it left, allowing it to enter the AOM at the Bragg angle once again and be deflected. This cancels the initial deflection, returning the beam along the original horizontal path and shifting the final frequency to $\omega_{2}=\omega_{0}+2 \Omega$. The vertical displacement from the $90^{\circ}$ angled mirror pair sends the beam back to the lens parallel but offset from the incident beam, causing the beam to pass through the AOM with some vertical angle. After the second pass through the $\mathrm{AOM}$, the frequency and vertically shifted beam can be picked off by a mirror and sent to the experiment.

\subsubsection{Nd:YAG Laser}

Ultraviolet, $355 \mathrm{~nm}$, light from a neodymium-doped yttrium-aluminum-garnet (Nd:YAG) laser was used to pump several dye amplifier stages. The Continuum Surelite III laser is 
a pulsed laser that consists of a $\mathrm{Nd}^{+3}$ doped YAG rod that is pumped by a flash lamp. A Q-switch consisting of a polarizer, a $\lambda / 4$ plate, and a Pockels cell determine when the laser light is allowed to build up within the laser cavity. A timing circuit waits until the rod is at maximum gain (around $233 \mu$ s after the flash lamp fires) and then applies $3600 \mathrm{~V}$ to the Pockels cell. This switches the birefringence of the Pockels cell producing a $\lambda / 4$ waveplate, allowing light to oscillate in the laser cavity (see Figure 2.4). After a few passes through the Nd:YAG rod, the high power laser light exits the cavity in a $5 \mathrm{~ns}, 1064 \mathrm{~nm}$ pulse. Upon exiting, the light passes through a a second harmonic generation (SLD) and third harmonic generation (SLT) module, producing the second and third harmonic of the light (532 nm and $355 \mathrm{~nm}$, respectively) [2]. Both the fundamental and second harmonic are dumped into a beam block while the ultraviolet third harmonic is used to pump a pair of dye amplifiers.

\subsubsection{Dye Amplifier}

The $10 \mathrm{~ns}$ light pulses sliced from the Toptica TA/DL-SHG pro laser are amplified using a transverse dye amplifier [61]. This dye amplifier uses a dilute solution of Coumarin 480 (120 mg per liter of methanol) as the gain medium to amplify the seeded $482 \mathrm{~nm}$ light. Ultraviolet pump light from the Surelite III Nd:YAG laser $(355 \mathrm{~nm})$ is focused on to a dye cell by a cylindrical lens. This light excites a narrow region of dye within the cell. The seed light from the Toptica laser passes through the excited region and stimulates the emission of more $482 \mathrm{~nm}$ light amplifying the seed. Though a pulse length of $10 \mathrm{~ns}$ has a nominal bandwidth of $100 \mathrm{MHz}$, additional spectroscopic measurements indicate a bandwidth of $\sim 150 \mathrm{MHz}$. 
(a)

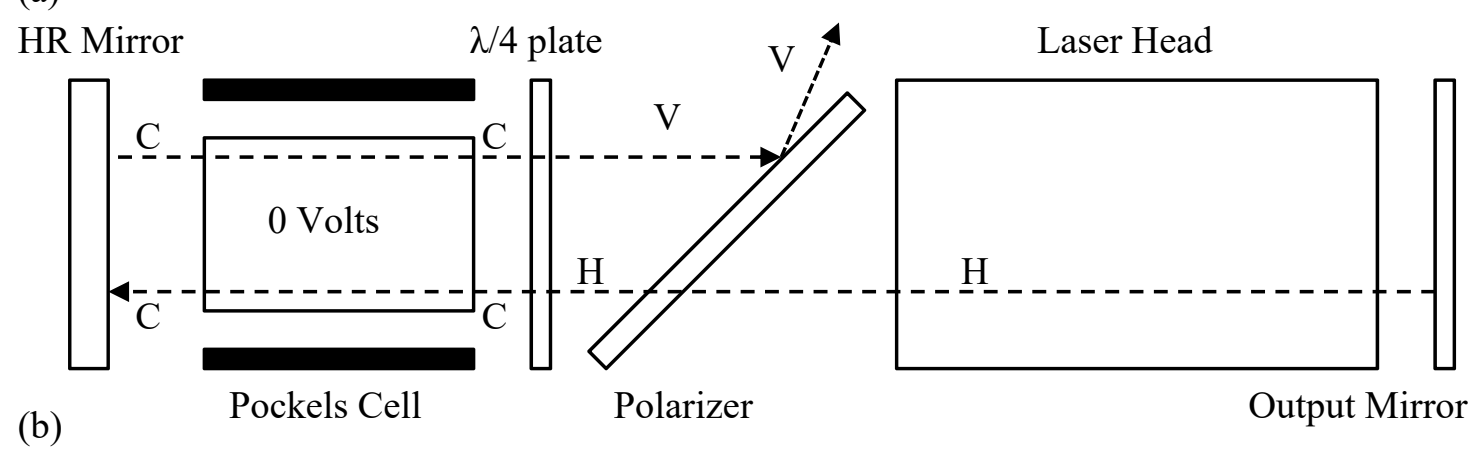

(b)

$\lambda / 4$ plate $\quad$ Laser Head

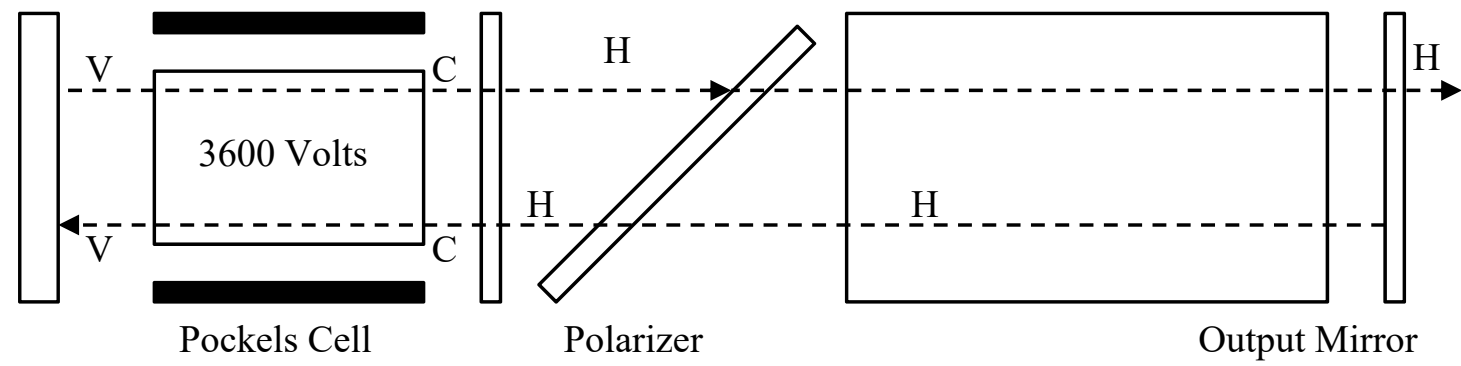

Figure 2.4: Schematic of the Surelite III Nd:YAG laser. The labels H, C, and V represent the horizontal, circular, and vertical polarizations of the light. Not shown here are the SLD and SLT modules that produce the second harmonic $532 \mathrm{~nm}$ light and the third harmonic $355 \mathrm{~nm}$ light, respectively. (a) The voltage across the Pockels cell is $0 \mathrm{~V}$ such that the polarization is only rotated by $\lambda / 4$ after each pass through the $\lambda / 4$ plate and the light is rejected by the polarizer. (b) The voltage across the Pockels cell is $3600 \mathrm{~V}$, creating another $\lambda / 4$ rotation on each pass through the Pockels cell. The net effect of the Pockels cell and waveplate is zero retardation, allowing the light to pass through the polarizer and the cavity to lase [2]. 


\subsection{Magneto-Optical Trap (MOT)}

The main apparatus in these experiments and the source of the atoms that we study is the magneto-optical trap (MOT). The MOT combines techniques of magnetic and optical trapping along with laser cooling to cool and confine atoms at temperatures in the microKelvin regime. A tool that has become the primary workhorse for many cold atom experiments, the MOT is a robust trap that does not depend on precise balancing of the counter-propagating beams or high degrees of polarization [3]. The trap can be run with a vacuum chamber at room temperature and either air or water cooled coils to produce the magnetic field [4]. Additionally, methods have been developed to allow room temperature gases to be cooled and trapped directly by MOT's [62]. This allows the vacuum chamber to be small and easy to build. The following section will include a brief overview of the theory behind the MOT, a brief discussion of our implementation and characterization of the MOT, and an update on recent changes and measurements made with our MOT. The initial implementation and characterization was done by Mary Kutteruf and is covered extensively in her dissertation [5]

\subsubsection{Theory of Operation}

The first attempts to cool and trap gases happened in one, two, and three dimensions. In three dimensions, work by Chu et al. produced some of the first "optical molasses" in which optical techniques were used to cool, i.e. slow, the atoms down to the temperature limit of Doppler cooling, the Doppler limit [63]. However, since there was no restoring force involved here the atoms were merely cooled and not contained. Following that, temperatures 
below the Doppler limit were measured in optical molasses experiments [64]. This major breakthrough led to the development of polarization gradient cooling theories that allow for sub-Doppler limit temperatures and new experimental techniques to confirm them [65-68].

While sub-Doppler temperatures can be reached in optical molasses through polarization gradient cooling techniques, these techniques do not yet provide confinement of the atoms. For that, we will need to introduce a weak magnetic field. To understand the theory behind the MOT, it is helpful to look at it in one dimension first. In a simple one dimensional trap, we consider an atom that has a ground state with total angular momentum $J_{g}=0$ and an excited state with total angular momentum $J_{e}=1$. The excited state can be further broken down in to three magnetic sublevels $M_{e}= \pm 1,0$. With the addition of a magnetic field $B(z)=A z$, the energy of the sublevels of the excited state will split due to the Zeeman effect as $\Delta E=\mu M_{e} B=\mu M_{e} A z$. A schematic drawing of the energy levels in the one dimensional MOT can be seen in Figure 2.5.

Due to the Zeeman shift, the energies of the excited state can now be tuned as a function of field and position. Excitation from the ground state to the excited state is provided by a pair of counterpropagating beams of opposite circular polarization $\left(\sigma_{+}\right.$and $\left.\sigma_{-}\right)$. When reddetuned by some amount $\delta$, the beams are nearer to resonant with one of the $M_{e}$ states. For $z>0$, the $\sigma_{-}$beam will scatter more light off of the atoms than the $\sigma_{+}$beam, pushing them towards the center of the trap. For $z<0$, the opposite case is true and the $\sigma_{+}$beam pushes the atoms towards the center of the trap. This can be easily expanded to a three dimensional MOT using 3 pairs of beams perpendicular to each other and any $J_{g} \rightarrow J_{e}=J_{g}+1$ transition $[3,4]$. The magnetic field for the 3D MOT is provided by a pair of anti-Helmholtz coils and a schematic of the 3D MOT can be seen in Figure 2.6. 


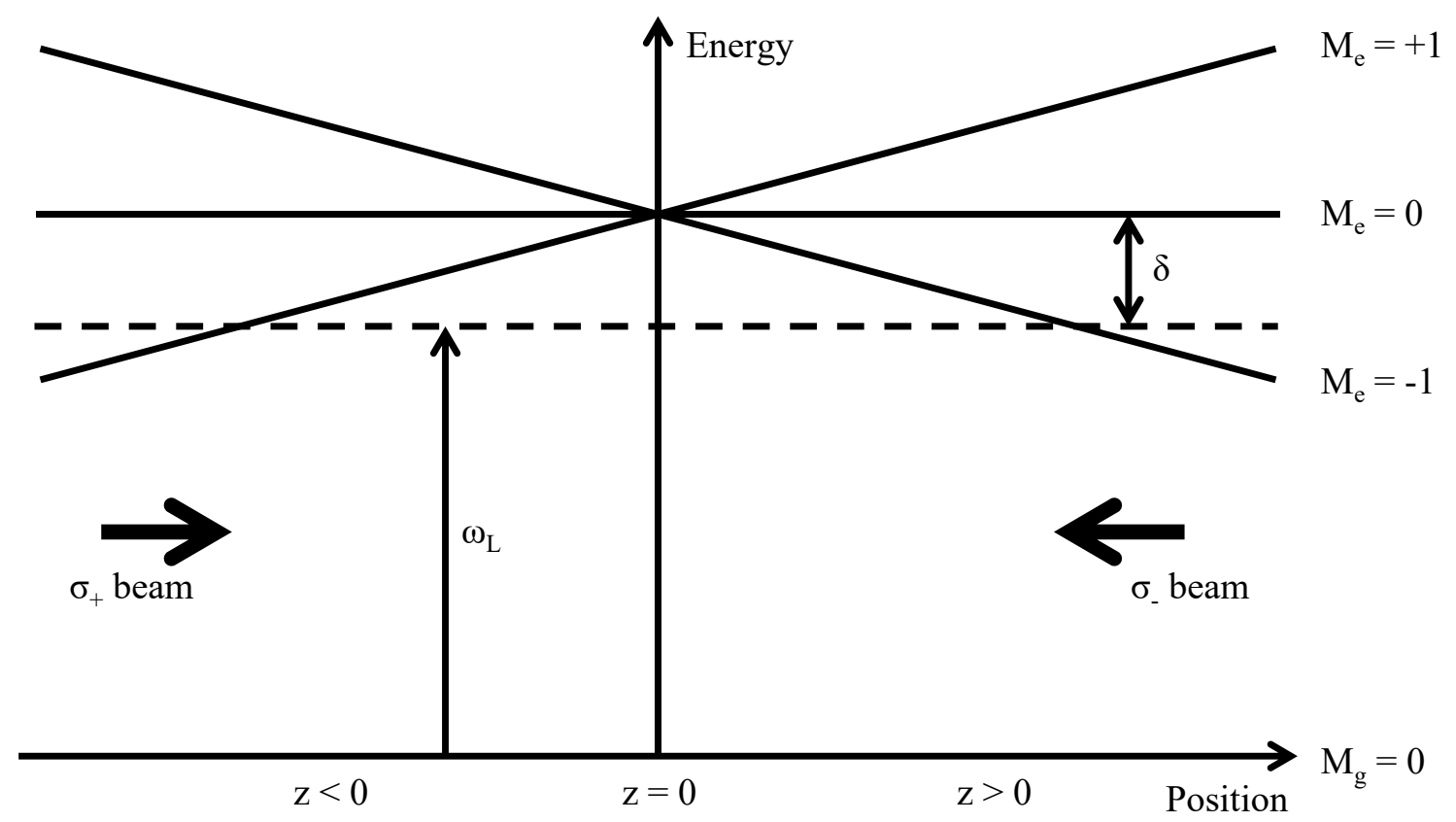

Figure 2.5: One dimensional energy level schematic of the MOT. The energy of excited state $e$ is split by the Zeeman effect into three sublevels $M_{e}=0, \pm 1$ for $J_{e}=1$. Counterpropagating beams with opposite circular polarization $\sigma_{+}$and $\sigma_{-}$are given a red-detuning $\delta$. Atoms at $z>0$ are more likely to scatter light from the $\sigma_{-}$beam while atoms at $z<0$ are more likely to scatter light from the $\sigma_{+}$beam. Therefore, the atoms outside of the center of the trap will be pushed towards the center. [3] 


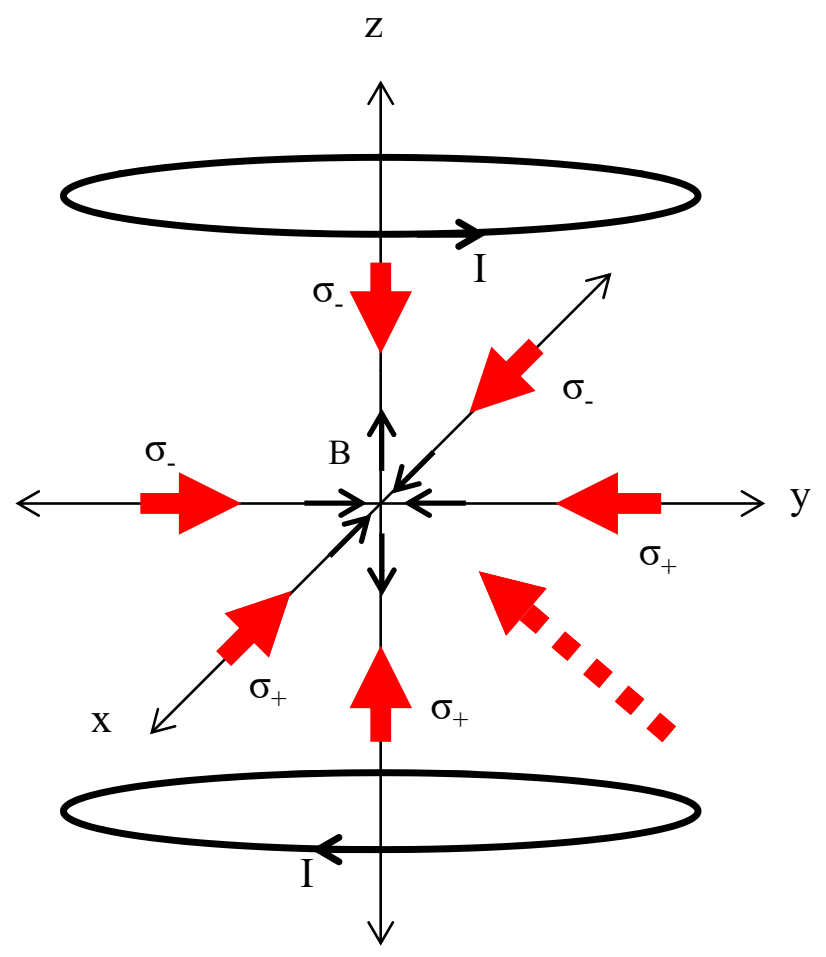

Figure 2.6: Schematic representation of the 3D MOT. The magnetic field is provided by a pair of anti-Helmholtz coils represented by the dark rings at the top and bottom of the schematic. An arrow on each shows the direction of the current. The thick black arrows on the axes show the direction of the magnetic field near the center of the trap. The large red arrows are the three pairs of counterpropagating beams with their polarizations labeled $[4,5]$. The large, dashed red arrow is the re-pump beam that is used to prevent pumping to the $5 s_{1 / 2}, F=2$ "dark" ground state as discussed in Section 2.2.2. 


\subsubsection{Implementation}

With a high background pressure, hot, or fast moving, atoms can kick cold atoms out of the MOT faster than the MOT can pull atoms in [69]. This requires a system with low background pressure ( $P_{\text {background }}<1 \times 10^{-8}$ torr) that operates in the ultra-high vacuum (UHV) regime for the MOT to form. The chamber is operated at $<5 \times 10^{-9}$ torr, typically in the $1 \times 10^{-10}$ torr to $2 \times 10^{-9}$ torr range. The vacuum chamber itself is comprised of a stainless steel spherical cube system from Kimball Physics Inc. Attached to the spherical cube is a valve for connecting to a roughing pump, a TiTan $20 \mathrm{~S}$ ion pump from Gamma Vacuum, and various high and low voltage feedthroughs. Housed within the chamber are Rubidium getters, a nude ion gauge, and a micro-channel plate (MCP) detector. All of the windows, four-way crosses, six-way crosses, and previously mentioned additions are connected to the chamber using conflat $(\mathrm{CF})$ seals.

To achieve UHV, we first rough pump the system using a turbo pump backed by a dry scroll roughing pump. The use of this roughing method is a change from the sorption pump method described in [5]. Once below $10^{-4}$ torr, the ion pump may be turned on, though we generally rough pump to lower pressures to increase the life of the ion pump. After being opened to atmosphere, the vacuum system must be baked to remove water and other contaminants from the walls. This is done by wrapping the chamber in heater tape, resistive straps that heat up when a voltage is applied, and covering the chamber with aluminum foil. The temperature of the chamber is raised to between $100{ }^{\circ} \mathrm{C}$ and $170{ }^{\circ} \mathrm{C}$. Care must be taken to not exceed approximately $200{ }^{\circ} \mathrm{C}$ as this will exceed the design specifications of the various elements on the chamber, particularly the windows and the magnets used in the ion pump. For additional reduction in the pressure, the ion pump itself may be baked as 
well as the chamber. To bake the ion pump, remove the pole piece that contains the magnets (black case) and remove the power cable. The ion pump can then be wrapped in heater tape and foil and heated up to $200{ }^{\circ} \mathrm{C}$.

The ion pump may occasionally show a rapidly changing pressure not seen when the ion gauge within the MOT chamber is on. This rapidly changing pressure is often indicative of a current leak between the cathode and the anode of the ion pump. To get rid of the current leakage, the ion pump may be removed from the vacuum chamber and rinsed with deionized water. This is done simply by pouring the deionized water into the port where the ion pump connects to the vacuum chamber.

We monitor the pressure within the chamber using a Bayard-Alpert ionization gauge controlled by a Varian senTorr gauge controller. The nude ionization gauge is placed directly in the chamber and can measure pressures down to $2 \times 10^{-11}$ torr. It measures the pressure by using a hot filament to emit electrons towards a wire grid. The electrons ionize background gas along the way and those ions are accelerated towards a collection wire. The current measured on the collection wire is proportional to the pressure of the background gas in the chamber. Since the ion gauge creates electrons and ions to detect the background pressure, we cannot use the ion gauge while running our experiments. The ion pump itself can also be used as an ion gauge where the current measured on the cathodes within the ion pump act similarly to the collection wire in the ion gauge. This provides a rough measurement that is generally a factor of 5 below the pressure measured with the ion gauge in our system.

To introduce the Rubidium atoms into the vacuum chamber, we use an alkali metal dispenser (AMD) from SAES Getters. The AMD uses anhydrous Rubidium metal salts 
of chromic acid with a reducing agent that evaporate when a DC current is applied, creating atomic Rubidium. By controlling the applied DC current, the background Rubidium pressure, and thus the amount of Rubidium accessible to the MOT, is controlled [70]. The AMD is typically operated at currents between $2.0 \mathrm{~A}$ and $2.5 \mathrm{~A}$ for a low and high density MOT, respectively. As the AMD ages, the output of Rubidium atoms is reduced and can be compensated for by increasing the current accordingly.

Many different methods exist to create the trapping potential used in the MOT [71]. For our implementation, we use a pair of field coils in an anti-Helmholtz configuration to provide the magnetic gradient used in the experiment. The coils are connected in series such that the current flows in opposite directions. The magnetic field created by these field coils can be computed by assuming we have two rings of current with radii $R$ and separation $2 A$. Close to the midpoint of the coils, the magnetic field can be approximated by

$$
\begin{gathered}
B_{z}(z, \rho)=\sum_{n=0} b_{n} B_{z n} \approx b_{1} z+b_{3}\left(z^{3}-\frac{3}{2} z \rho^{2}\right) \\
B_{\rho}(z, \rho)=\sum_{n=0} b_{n} B_{\rho n} \approx-\frac{b_{1} \rho}{2}+b_{3}\left(-\frac{3 \rho z^{2}}{2}+\frac{3 \rho^{2} z}{8}\right)
\end{gathered}
$$

where $z$ is measured along the axis of the coils, $\rho$ is measured radially from the center of the coils, and the origin is defined as the midpoint between the coils. The constants $b_{1}$ and $b_{3}$ are defined as

$$
\begin{gathered}
b_{1}=\frac{3 \mu I A R^{2}}{\left(R^{2}+A^{2}\right)^{5 / 2}} \\
b_{3}=\frac{5\left(4 A^{2}-3 R^{2}\right)}{6\left(R^{2}+A^{2}\right)^{2}} b_{1} .
\end{gathered}
$$


For an anti-Helmholtz configuration where $A=R / 2$, we have $b_{3} \ll b_{1}$ so

$$
\frac{\partial B}{\partial z}=b_{1}=-2 \frac{\partial B}{\partial \rho}
$$

showing that the field varies linearly in all directions away from the center, with the radial gradient twice that of the gradient along the coil axis [5,71,72]. Our coils consist of 114 turns of Polyurethane-Nylon coated copper wrapped around a $15 \mathrm{~cm}$ diameter aluminum and plastic frame. Typical currents of $10 \mathrm{~A}$ provide an on-axis magnetic field gradient of $\sim 15$ Gauss/cm [5].

For the magneto-optical trap, we use ${ }^{85} \mathrm{Rb}$ provided by the Rubidium getters. The structure of ${ }^{85} \mathrm{Rb}$ is a bit more complicated than the structure shown in Section 2.2.1. The two states that we use for our trap are the $5 s$ and $5 p$ states of Rubidium. These states exhibit a fine-structure splitting with the $5 s$ state having a total angular momentum $j=1 / 2$ and the $5 p$ state being split into two states with total angular momentum $j=1 / 2,3 / 2$. With the addition of the nuclear spin $\vec{I}$ of the atom, the states are further split into hyperfine states of the from $\vec{F}=\vec{I}+\vec{J}$. With a nuclear spin of $I=5 / 2$, the $5 s_{1 / 2}$ state splits into two hyperfine states $F=2,3$ and the $5 p_{3 / 2}$ state splits into four hyperfine states $F=1,2,3,4$ (see Figure 2.7).

For an ideal trapping cycle, we require closed transitions between some ground state and an excited trapping state. For the ground state we use the $5 s_{1 / 2}, F=3$ state and for the trapping state we use the $5 p_{3 / 2}, F=4$ state. Unfortunately, there are a small number of atoms that make a transition to the nearby $5 p_{3 / 2}, F=3$ state which is only $120 \mathrm{MHz}$ away from the $F=4$ state. The atoms in the $F=3$ state can now decay down to the $5 s_{1 / 2}$, $F=2$ state, which is not accessible by our trapping lasers. This makes the $5 s_{1 / 2}, F=2$ 


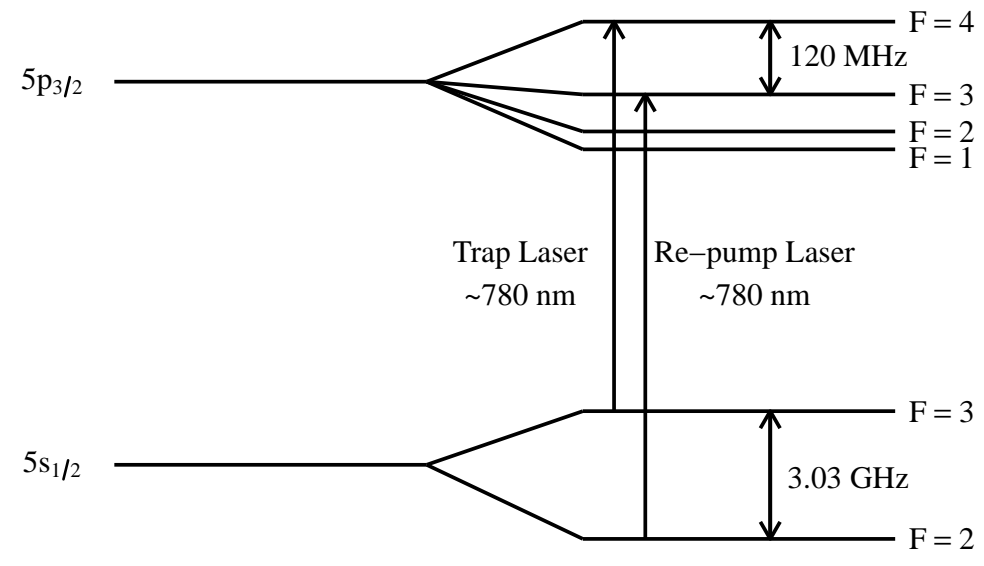

Figure 2.7: Schematic diagram of the energy levels of ${ }^{85} \mathrm{Rb}$ showing the trapping and repumping transitions along with the hyperfine structure of the $5 s_{1 / 2}$ and $5 p_{3 / 2}$ states (energy levels are not to scale).

state "dark", and as more and more atoms flow into the dark state, our trap empties. To prevent the destruction of our trap, we introduce a weakly-driven second transition called a re-pumping transition. The re-pumping transition will pump atoms in $5 s_{1 / 2}, F=2$ up to the $5 p_{3 / 2}, F=3$ state. From there, the atoms can reenter the trapping cycle $[5,7,73]$.

The trap and re-pump lasers used to excite the atoms to the $5 p_{3 / 2} F=4$ and 3 states, respectively, are provided by two Vortex Stablewave diode lasers described in Section 2.1.1. The frequencies of the lasers are stabilized to the side of a resonance feature produced using Doppler-free saturated absorption spectroscopy and locked using a proportional-integralderivative (PID) feedback lock. The saturated absorption spectrum is obtained using a pump-probe measurement in a room-temperature Rubidium vapor gas cell. For both the trap and re-pump lasers about $4 \%$ of the beam is picked off and sent through the gas cell. 
This pump light is enough light to saturate the transition of interest. After passing through the gas cell, a beam splitter picks off a small amount of the pump light, turning it into the probe beam, and sends it back through the cell. To better understand saturated absorption spectroscopy, assume that there is a pump beam with detuning $\delta=\omega-\omega_{0}$ from some atomic resonance with frequency $\omega_{0}$ and that it is traveling in the $+\hat{z}$ direction. The only atoms that will be excited by the pump beam will be those with velocity $v_{z}=\delta / k_{0}$, where $k_{0}$ is the wavenumber of light with $\delta=0$. The returning pump beam will be traveling in the $-\hat{z}$ direction and will only excite atoms with velocity $v_{z}=-\delta / k_{0}$. Thus, the pump and probe beams access two different groups of the atoms. The only frequency at which the pump and probe beams access the same group of atoms is when $\delta=0$. At that frequency fewer ground state atoms are available to absorb light from the probe beam, leading to a reduction in its absorption. As the frequency of the laser is scanned, a dip at $\omega=\omega_{0}$ will appear and is usually called a Lamb dip [74].

For the case of several closely spaced resonances, there is the additional complexity of cross-over features. These are additional dips in the absorption spectrum that occur at frequencies directly between two resonances. In this case, the detuning of the beam is $\delta_{1} / 2=$ $\left(\omega-\omega_{1}\right) / 2=-\left(\omega-\omega_{2}\right) / 2=-\delta_{2} / 2$ where $\omega_{1}$ and $\omega_{2}$ are two neighboring resonances. In this case, the pump beam will access the group of atoms at velocity $v_{z}=\delta_{1} /\left(2 k_{0}\right)$ and the probe beam will access the group of atoms at velocity $v_{z}=-\delta_{1} /\left(2 k_{0}\right)=\delta_{2} /\left(2 k_{0}\right)$. Thus, the pump and probe beams are again accessing the same velocity group, producing a dip in the absorption of the probe beam at $\omega=\omega_{2}-\omega_{1}$ [74]. We use the $5 p_{3 / 2}, F=4$ to $5 p_{3 / 2}, F=3$ cross-over resonance dip in the PID feedback lock for the trap laser. An AOM is used to shift the frequency of the trap laser saturated absorption pump beam after 
it has been split off from the main beam. This allows us to add a detuning to the pump of $\delta=-36 \mathrm{MHz}$. With the pump beam locked to the side of the $5 p_{3 / 2}, F=4$ to $5 p_{3 / 2}, F=3$ cross-over resonance near the $5 s_{1 / 2}, F=3$ to $5 p_{3 / 2}, F=4$ resonance, the trap laser is red detuned by approximately $20 \mathrm{MHz}$, or approximately 3 times its natural line width.

Within the vacuum chamber are two pairs of parallel rods used to provide electric fields for both ionization and energy tuning. The rods in each pair are separated by $2.29 \mathrm{~cm}$ and the pairs are separated by $1.52 \mathrm{~cm}$ from one another. The uniformity and strength of the electric field is detailed in Section 2.2.3. For a more detailed description of our implementation of the MOT, see Mary Kutteruf's doctoral dissertation [5].

\subsubsection{Characterization, Updates, and Modifications}

In this section, we characterize the atom trap and discuss modifications that have been made to the MOT. Many of these measurements were made by Mary Kutteruf and are detailed in her dissertation [5]. She measured the temperature of the MOT to be $67.5 \mu \mathrm{K}$ and the size of the MOT to be approximately $0.4 \mathrm{~mm}$. The temperature of the MOT was found to be quite independent of density and trap/re-pump laser power. The size of the MOT is continuously monitored using a CCD camera, which also monitors the density of the atoms in the MOT. The size of the MOT is relatively constant as a function of getter current, and thus density, below a maximum density of $\simeq 1.2 \times 10^{10} \mathrm{~cm}^{-3}$. For higher getter currents after reaching a density of $\simeq 1.2 \times 10^{10} \mathrm{~cm}^{-3}$, the radius of the MOT grows while remaining at nearly constant density.

To measure the density of atoms and the number of atoms in the MOT, we use an all optical method detailed in the dissertations of Anderson, Han, and Kutteruf $[5,72,75]$. The 
number of atoms in the $5 p_{3 / 2}, F=4$ trap state is proportional to the total light scattered by them. Since saturation of the trapping transition leads to half of the atoms being in the trap state at any one time, we can make the estimate that one atom scatters one photon per two lifetimes of the excited state. Thus, the power emitted by one atom is

$$
P_{1}=\frac{h c}{\lambda} \frac{1}{2 \tau}
$$

where $h$ is Planck's constant, $c$ is the speed of light, $\lambda$ is the wavelength of the scattered photon $(780 \mathrm{~nm})$, and $\tau$ is the natural lifetime (approximately $27 \mathrm{~ns}$ for the trapping transition). Therefore, the total number of atoms is

$$
N_{A}=\frac{P_{\text {Total }}}{P_{1}}
$$

where $P_{\text {total }}$ is the total power of the fluorescence emitted by the MOT. The fluorescence of the MOT is monitored using a CCD camera and is calibrated by imaging the MOT fluorescence onto a power meter. A 2-inch diameter, $f=+100 \mathrm{~mm}$ lens is placed a distance $d_{o}=17 \mathrm{~cm}$ from the MOT. If we assume that each glass-air interface has a transmissivity of $\mathcal{T}=0.96$ then the total power of the MOT is

$$
P_{\text {total }}=\frac{P_{\text {meter }}}{\mathcal{T}^{4}} \frac{4 d_{o}^{2}}{r^{2}}
$$

where $r$ is the radius of the lens and $r \ll d_{0}$ [5]. We discovered that due to the spherical aberrations of the $f=+100 \mathrm{~mm}$ lens, not all of the light reached the detector. To get a better understanding of the effective radius of the lens, we applied a 1-inch diameter mask 
that acted as an aperture for the lens. This made the effective radius $1 / 2$ an inch. With the application of the mask we saw a decrease in the power from the MOT by a factor of 2 instead of the factor of 4 that we would expect for dropping the radius of the lens by a factor of 2. The power at high getter current (high density) was used to determine the calibration between the power meter and the $\mathrm{CCD}$ camera. The $\mathrm{CCD}$ camera was used alone for all later density and atom number calculations. The calibration between the power meter and the camera is $N_{A}=S_{\text {total }} \cdot 1.19 \times 10^{6}$, where $S_{\text {total }}$ is the total integrated signal from the CCD camera. For the experiments described in this dissertation, the atoms are excited to a single Rydberg state. Assuming saturation of both the $5 s_{1 / 2} \rightarrow 5 p_{3 / 2}$ and $5 p_{3 / 2} \rightarrow n l$ Rydberg transition, one-third of all the atoms end up in the Rydberg state. In the experiments we achieve Rydberg densities of $2 \times 10^{8} \mathrm{~cm}^{-3} \leq \rho \leq 4 \times 10^{9} \mathrm{~cm}^{-3}$.

For the transition $5 s_{1 / 2}, F=3 \rightarrow 5 p_{3 / 2}, F=4$, we have a strong continuous wave (cw) laser that stays on during our measurements. We can now think of the ground state and trap state as an "atom-laser" system or a dressed atom system. Let us assume that we have three states $a, b$, and $c$. We can treat $a$ as the ground state of the MOT and $b$ as the trap state of the MOT with energy separation $\omega_{0}$. State $c$ is a higher lying state with energy separation $\omega_{1}$ from state $b$, that can be laser excited by a second laser from state $b$. Moving to the dressed atom picture we have a Hamiltonian of the system

$$
H=H_{A}+H_{L}+V_{A L}
$$

where $H_{A}$ is the Hamiltonian for the atom, $H_{L}$ is the Hamiltonian for the laser, and $V_{A L}$ is the atom-laser interaction [76]. We can now write out the dressed atom states $|a, N+1\rangle$, $|b, N\rangle$, and $|c, N\rangle$ where $\mathrm{N}$ is the (large) number of photons incident on the atom from the 
strong cw laser. As can be seen in Figure 2.8, the total energy of states $|a, N+1\rangle$ and $|b, N\rangle$ are $\delta=\omega_{L}-\omega_{0}$ where $\omega_{L}$ is the frequency of the laser. When we include the atom-laser interaction, our states $|a, N+1\rangle$ and $|b, N\rangle$ split into two new states $|1\rangle$ and $|2\rangle$ which both contain an admixture of $|b, N\rangle$ and are split by energy $\Omega=\sqrt{\Omega_{1}^{2}+\delta^{2}}$, where $\Omega_{1}$ is the Rabi frequency of the atom-laser interaction. We can now use a beam with frequency $\omega_{L}^{\prime}$ to excite the atom from the states $|2\rangle \rightarrow|c, N\rangle$ and $|1\rangle \rightarrow|c, N\rangle$. By scanning the frequency $\omega_{L}^{\prime}$ we get two peaks, the so-called Autler-Townes doublet, which are separated by a frequency $\Omega$. Figure 2.9 show the results of spectroscopic measurements of the $32 p_{3 / 2}$ Rydberg state with the $32 p_{3 / 2},\left|m_{j}\right|=1 / 2$ state as a bold line and the $32 p_{3 / 2},\left|m_{j}\right|=3 / 2$ state as a thin line. The $32 p_{3 / 2}$ state is excited by a $3 \mu \mathrm{s}, 482 \mathrm{~nm}$ laser pulse from the $5 p_{3 / 2}$ trap state, which is pumped from the $5 s_{1 / 2}$ state by the trap lasers with a Rabi frequency $\Omega_{1} \approx 16 \mathrm{MHz}$ and a detuning $\delta \approx 20 \mathrm{MHz}$ to the red of the $5 p_{3 / 2}$ state. As can be seen in the bold line in Figure 2.9 , there are two primary peaks of unequal heights separated by $\Omega \approx 25 \mathrm{MHz}$. This is due to the detuning $\delta$ of the laser driving states $a$ and $b$. For a laser of detuning $\delta=0$, the peaks would be of equal height. The Autler-Townes doublet structure seen here will have to be accounted for in all of the following experiments [76]. The small satellite features, left of the main $m_{j}=1 / 2$ peak and right of the main $m_{j}=3 / 2$ peak in Figure 2.9, are due to excitation of the $m_{j}=3 / 2$ and $m_{j}=1 / 2$ states, respectively.

The electric field produced by the field rods described earlier is calculated to be

$$
\vec{E}(x, y)=-E_{0}\left\{\left[\frac{8 x_{0}^{2}}{\left(x_{0}^{2}+y_{0}^{2}\right)^{2}}-\frac{2}{x_{0}^{2}+y_{0}^{2}}\right] x y \hat{x}+\left[1+\frac{4\left(x_{0}^{2} x^{2}+y_{0}^{2} y^{2}\right)}{\left(x_{0}^{2}+y_{0}^{2}\right)^{2}}-\frac{\left(x^{2}+3 y^{2}\right)}{x_{0}^{2}+y_{0}^{2}}\right] \hat{y}\right\}
$$

where $E_{0}=|\vec{E}(0,0)|=2 \lambda y_{0} / \pi \epsilon_{0}\left(x_{0}^{2}+y_{0}^{2}\right)$ is the magnitude of the electric field at the 


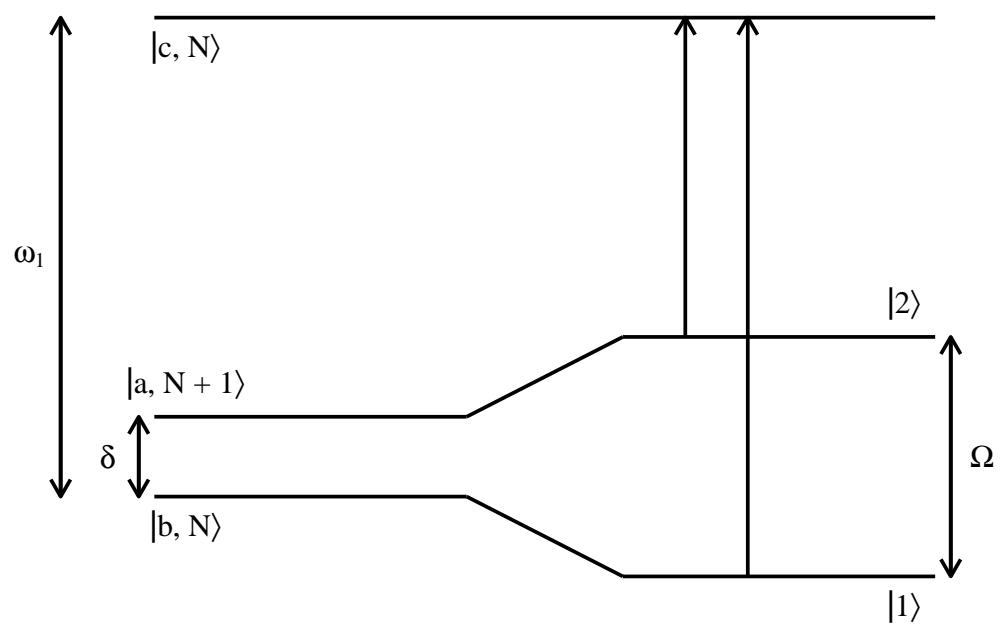

Figure 2.8: The energy levels for a three-level dressed atom. The energy levels on the left side are the energies for states $a, b$, and $c$ in the dressed atom picture without interaction. States $|1\rangle$ and $|2\rangle$ are the states with the atom-light interaction. State $c$ remains unchanged as the laser driving states $a$ and $b$ is far off resonance for state $c$. The energy gap between states $b$ to $c$ is $\omega_{1}$. 


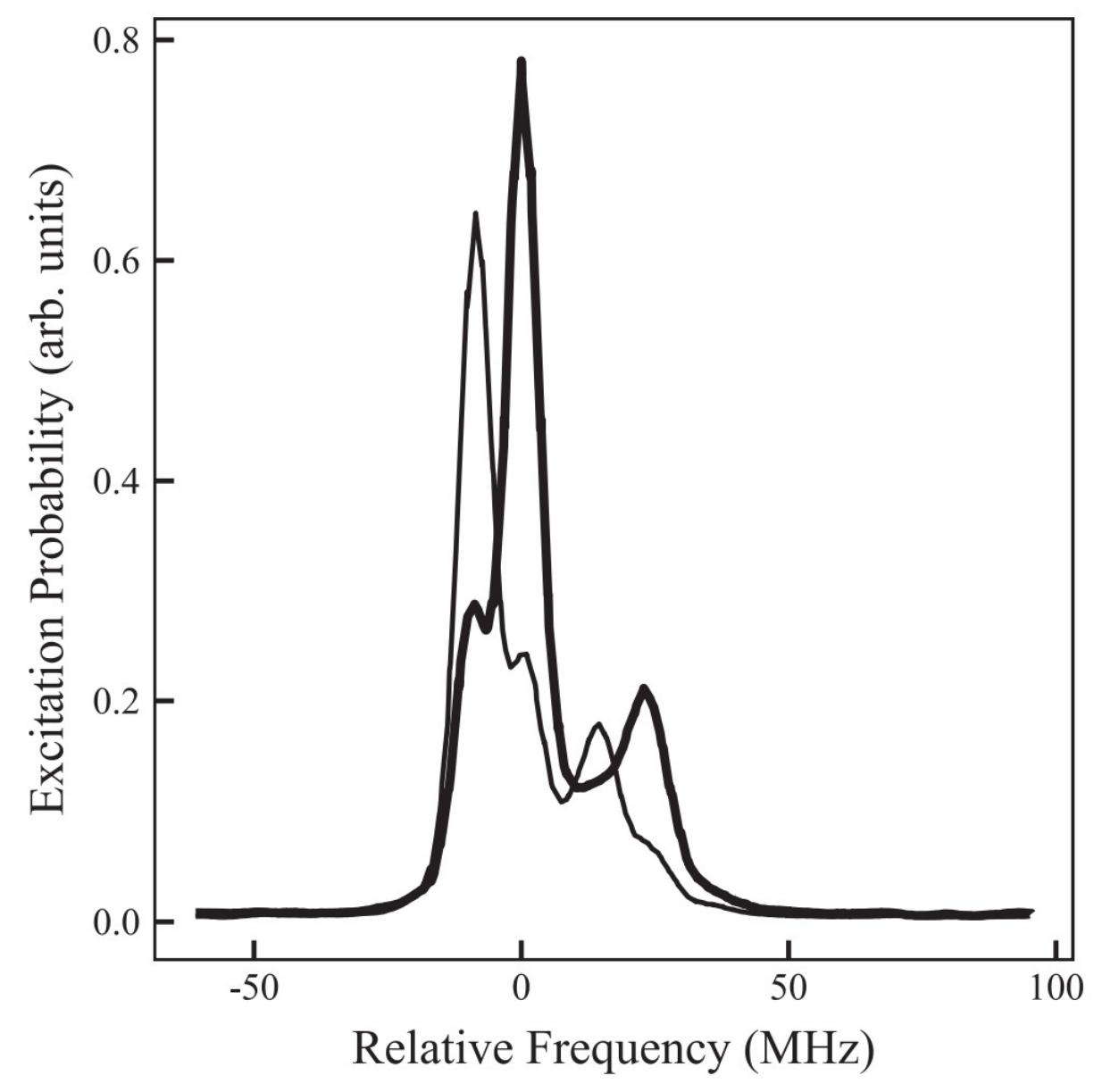

Figure 2.9: Spectroscopy of the $32 p_{3 / 2}$ Rydberg state of ${ }^{85} \mathrm{Rb}$. The bold line is primarily the $m_{j}=1 / 2$ state and the thin line is primarily the $m_{j}=3 / 2$ state. Both states show a primary peak and a smaller satellite peak consistent with an Autler-Townes doublet [6]. The small satellite features, left of the main $m_{j}=1 / 2$ peak and right of the main $m_{j}=3 / 2$ peak in, are due to excitation of the $m_{j}=3 / 2$ and $m_{j}=1 / 2$ states, respectively. 
center of the chamber and $x_{0}=1.145 \mathrm{~cm}$ and $y_{0}=0.76 \mathrm{~cm}$ are the distance of the rods from the center of the chamber. Over the size of the MOT $(x=0.05 \mathrm{~cm}, y=0.05 \mathrm{~cm})$, the change in the electric field is $|\vec{E}(x, y)-\vec{E}(0,0)| /|\vec{E}(0,0)|=0.47 \%$, showing that the electric field is remarkably uniform over the size of the MOT. We can treat the field across the MOT as being created by a pair of infinite parallel plates as

$$
\vec{E}=\frac{\Delta V}{d_{e f f}}
$$

where $\Delta V=V_{2}-V_{1}$ is the voltage difference between the rod pairs and $d_{e f f}$ is the effective separation. $V_{2}$ and $V_{1}$ are the voltages applied to the rod set closest and furthest from the MCP, respectively. To determine $d_{e f f}$, we measure the electric field at the location of the MOT using the splitting of the two $m_{j}$ states of the $32 p_{3 / 2}$ state. At low fields, the splitting between the states $\Delta(F)=E_{3 / 2}-E_{1 / 2}$ scales quadratically with field. The splitting of the states was measured by performing spectroscopy of the states as seen in Figure 2.9 and taking the difference in the peak locations. This experimental data was compared to a calculation of the energies of the two $m_{j}$ states (see Section 2.4), to determine the actual field in the MOT (see Figure 2.10). From this, we obtained an effective separation $d_{e f f}=3.771 \pm 0.002 \mathrm{~cm}$.

At zero electric field, the energy of the $m_{j}$ states should be degenerate and their splitting should be zero. Additionally, we should be unable to populate the $32 p_{3 / 2}$ state from $5 p_{3 / 2}$ because there will be no $s$ and $d$ character mixed into the $32 p_{3 / 2}$ state at zero electric field. Figure 2.10 shows the splitting of the $m_{j}$ states as a function of applied electric field. That the splitting does not go to zero at zero applied field indicates the existence of an additional offset field. We can determine the offset field parallel to the applied field direction by 


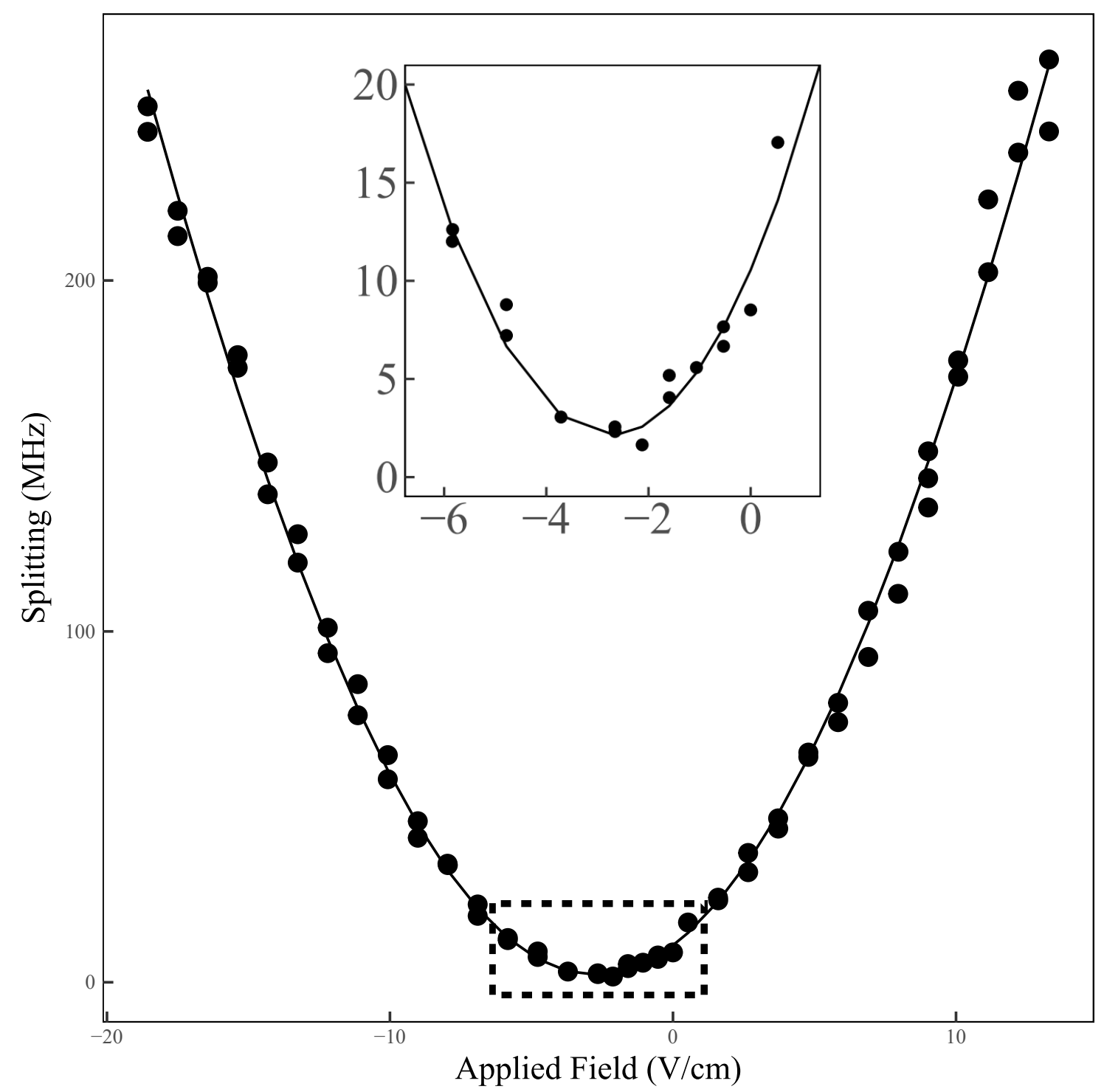

Figure 2.10: The energy splitting between the $32 p_{3 / 2}\left|m_{j}\right|=3 / 2$ state and the $32 p_{3 / 2}$ $\left|m_{j}\right|=1 / 2$ state. The points are experimental data collected by performing spectroscopy on the $m_{j}$ states. The solid line is a calculation of the difference between the $m_{j}$ states done by calculating the Stark shift for all of the states near to the $32 p$ state. The inset shows the points near the minimum of the splitting [6]. 
adjusting the applied field to find the minimum splitting. That this minimum splitting is not zero indicates the presence of an additional offset field perpendicular to the applied field which cannot be canceled by the field rods. These fields are likely due to field leakage caused by improper shielding of the micro-channel plate (MCP) detector used to measure the Rydberg states. We find that there is a $2.758 \pm 0.005 \mathrm{~V} / \mathrm{cm}$ offset field parallel to the field created by the rods. We also find that there is a $1.38 \pm 0.04 \mathrm{~V} / \mathrm{cm}$ field perpendicular to the field created by the field rods. The perpendicular offset field is added in quadrature to the applied field to determine the splitting of the $m_{j}$ states. The parallel offset field is also taken into account when calibrating the applied field. The effect of both offset fields can be clearly seen in Figure 2.10 and is discussed further in Chapter 3.

In addition to the characterization described above, several modifications were made to the MOT and the lasers used in the MOT. In February of 2015, a leak in the cooling structures surrounding the MOT magnetic field coils was detected. Damage to the aluminum cooling coil had occurred due to high water pressure. The hole was repaired using marine-grade epoxy and a paddle-wheel flow indicator was installed in the return line for regular pressure monitoring. To prevent high water pressure from occurring accidentally, the water to the MOT is shut off when the MOT is not in use and the flow indicator is monitored during MOT use, with an optimal flow velocity of $\sim 0.01 \mathrm{~m} / \mathrm{s}$.

Modifications were made to the beam path of the re-pump laser to accommodate an acousto-optic modulator for turning on and off the MOT during an experiment. This is done by sending the 1 st order diffracted beam from the AOM into the MOT. When the AOM is switched, the re-pump beam is removed from the MOT and atoms exit the trap over the course of approximately 1 second (see Chapter 5). 

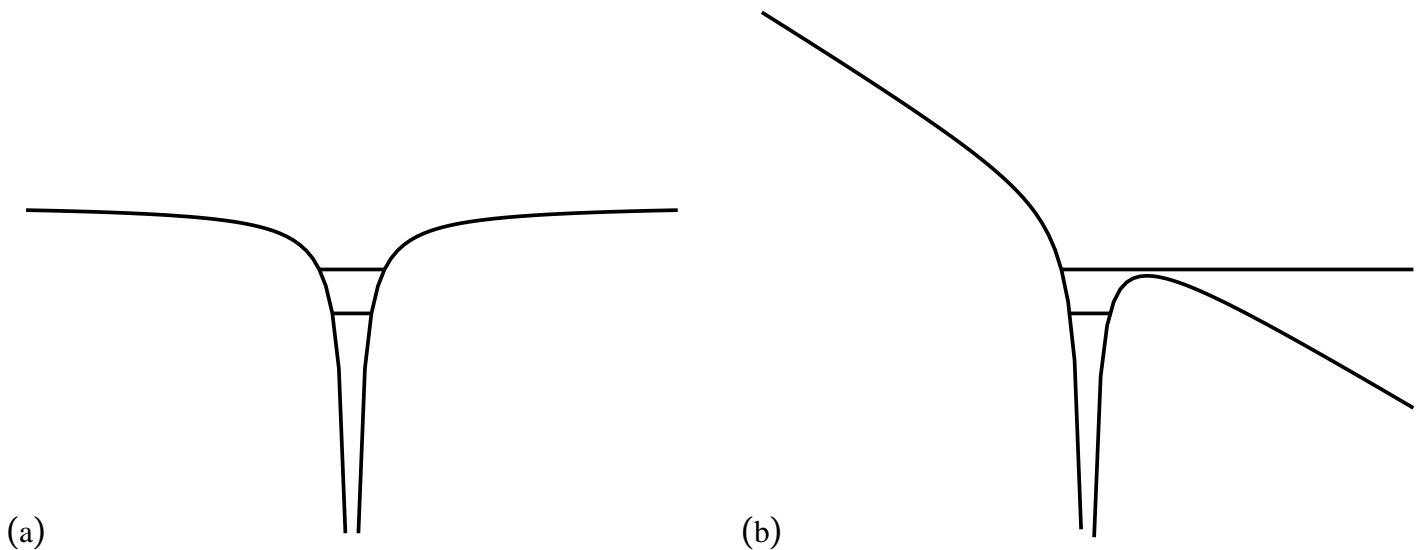

Figure 2.11: Schematic of a $1 / r$ potential with (a) no external electric field and (b) an external electric field $F$. The solid lines represent bound energy states of the electron. When the field $F$ is applied, the electron in the uppermost state is above the barrier and can escape from the potential.

\subsection{Electronics}

The experiments described in the following chapters use a number of different electronics for the production of electric fields, timing, detection, and frequency stabilization. The particular devices and methods used will be discussed here.

\subsubsection{Field Ionization}

Measurement of Rydberg states is achieved through state-selective field ionization (SSFI) $[8,77]$. With SSFI, we apply a strong electric field that ionizes Rydberg atoms. The resulting ions are then accelerated towards a micro-channel plate (MCP) detector (see Section 2.3.2). Assume that the Rydberg atom has a potential $V(r) \sim 1 / r$ (see Figure 2.11(a)). If an electric field $F$ is applied in the $z$ direction, then its potential will change as 
$F z$. The net potential tips downwards for positive $z$ and bound electrons with $z>0$ and energies greater than the potential maximum can escape to $z=\infty$ (see Figure 2.11(b)). To obtain state-selectivity, we can apply a "slow", $\sim 3 \mu$ s rising pulse to the field rods such that different energy electrons ionize at different times. By measuring the number of ions that are collected by the MCP detector as a function of time, we can distinguish Rydberg atoms in different initial states [8]. The field ionization pulse is applied to the pair of field rods furthest from the detector (closest to the pump) allowing for the acceleration of the ions towards the MCP detector.

\subsubsection{Micro-Channel Plate (MCP) Detector}

For detection of Rydberg atoms, we use a micro-channel plate (MCP) detector in a chevron configuration (see Figure 2.12). Ions produced by field ionization are accelerated toward the detector. Once they reach the detector, the ions hit the face of the MCP and cause a cascade of electrons through the micro-channels and out the back of the plate. The electrons are then amplified by a second MCP before being collected on a metal plate [78]. The resulting time-dependent current is used to determine when ions arrive. The time at which ions arrive is proportional to the field required to ionize the Rydberg atom, allowing us to distinguish between Rydberg atoms in different initial states.

\subsubsection{Electric Fields}

Several different electric field sources are used in the experiments described below. Static fields are created in the MOT using the HP 6207B DC Power Supply. This power supply provides between $0 \mathrm{~V}$ to $160 \mathrm{~V}$ at $0.0 \mathrm{~A}$ to $0.2 \mathrm{~A}$ [79]. This static field is added to 


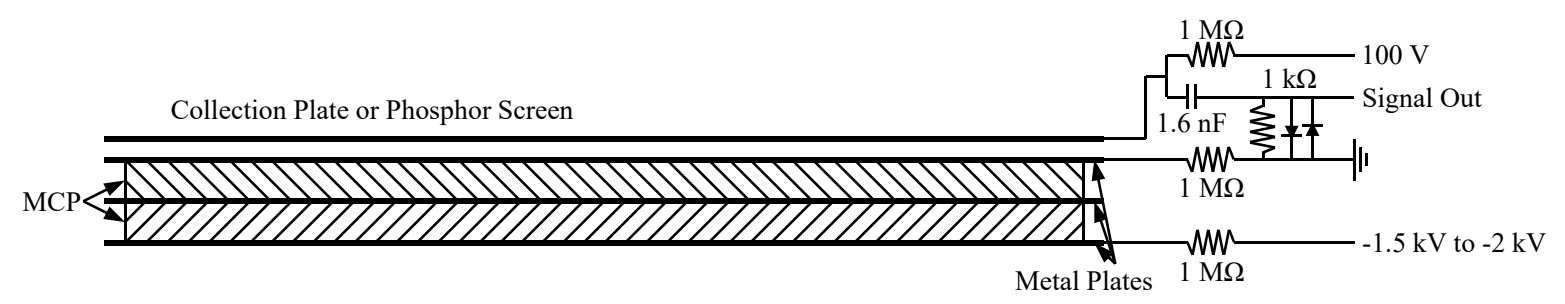

Figure 2.12: Schematic of the micro-channel plate (MCP) detector set up in a chevron configuration for collecting ions. The detector consists of two micro-channel plates sandwiched between three thin metal plates. For ion detection, the front plate is held between $-1500 \mathrm{~V}$ to $-2000 \mathrm{~V}$ and the back plate is held slightly above ground while the middle plate is allowed to float. The electrons emitted by the MCPs are collected on the collection plate which is held at $100 \mathrm{~V}$. The voltage on the back plate is measured as a function of time and indicates the relative number of ions hitting the detector versus time [5].

the field from the field ionization pulse and applied to the rods furthest from the detector. Pulsed fields are provided by a Tektronix Arbitrary Waveform Generator (AWG) 510. As the name indicates, the AWG creates user-definable waveforms with a max output of \pm 2 $\mathrm{V}$ into $50 \Omega$. With a clock speed of $1 \mathrm{GHz}$, rise/fall times of several nanoseconds are achievable [80]. To increase the output from the AWG, an AV-141F pulse amplifier module from Avtech Electrosystems was used. The amplifier is capable of providing $\times 10$ gain up to an output of $\pm 5 \mathrm{~V}$ into $50 \Omega$ with a rise/fall time of $4 \mathrm{~ns}$ and a bandwidth ranging from DC up to $75 \mathrm{MHz}[81]$.

\subsubsection{Delay Generators}

Timing synchronization is provided by two delay generators, the Stanford Research Systems DG 535 and DG 645. These delay generators each provide 4 independent outputs with a resolution of $5 \mathrm{ps}[82,83]$. The delay generators synchronize the laser pulses, electric fields, and data acquisition systems in the experiment. All of these are triggered at $15 \mathrm{~Hz}$, 


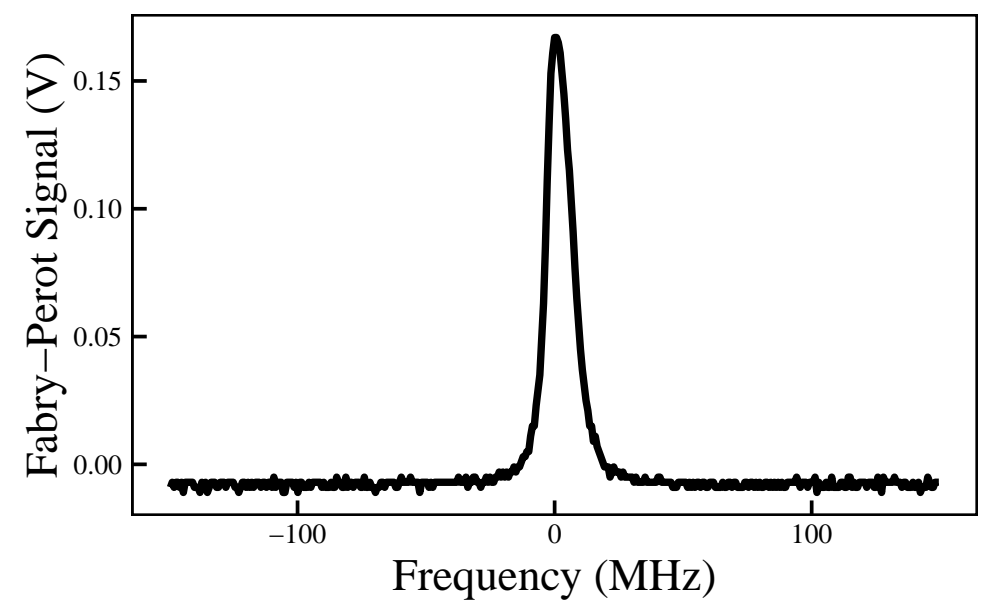

Figure 2.13: A sample trace of the signal from the Fabry-Pérot interferometer. The cavity length of the FPI is set such that the transmission through the cavity is approximately half of the maximum transmission at the frequency we wish to lock.

on every fourth cycle of the AC line. This allows all of the equipment in the laboratory to be synchronized to one another and eliminates the effect of $60 \mathrm{~Hz}$ pick-up on the trigger signals.

\subsubsection{Frequency Stabilization}

We use several methods to stabilize the frequencies of the diode lasers described in Section 2.1.1. For the diode lasers used as trapping and re-pumping lasers in the MOT, we use saturated absorption spectroscopy to implement a side-of-resonance lock using a proportional-integral-derivative (PID) feedback system (described in Section 2.2.2). For the Toptica diode laser used to excite the trapped atoms to Rydberg states, we use a Fabry-Pérot Interferometer (FPI) and a PID feedback system to implement a side-of-resonance lock for the $960 \mathrm{~nm}$ laser light from the master diode laser. 
The $960 \mathrm{~nm}$ infrared light from the TA-SHG Pro is sent to a Thor Labs SA200-3B FabryPérot Interferometer. The FPI contains a back reflector attached to a piezoelectric that allows for control of the cavity length. By scanning the length of the cavity, the spectrum of the laser will be measured (see Figure 2.13). Scanning of the cavity length is provided by the Thor Labs SA201 Spectrum Analyzer Controller. This controller provides either a triangle wave or sawtooth wave voltage to the piezoelectric in the FPI and allows for additional control over the length of the voltage sweep and the offset of the sweep. Additionally, the controller has a built in amplifier that can give $\mathrm{a} \times 1, \times 10$, or $\times 100$ amplification of the FPI signal. For the laser lock, we remove the voltage ramp from the SA201 Spectrum Analyzer Controller and use a Thor Labs TPZ001 Piezo driver to apply a static voltage from $0 \mathrm{~V}$ to $150 \mathrm{~V}$. To lock the diode laser, we first set the frequency of the laser using some external reference (often a Rydberg resonance). We then adjust the cavity length of the FPI using the TPZ001 Piezo driver to find the rising edge of the FPI signal and we set it to half-way up the peak. We then feed the FPI signal through the SA201 amplifier and send the amplified signal to the Toptica PID 110 (see Figure 2.14 for connection details). The PID 110 is a PID feedback controller with up to $1.5 \mathrm{MHz}$ bandwidth provided as part of the DC 110 supply electronics with the TA-SHG Pro.

As mentioned above, these locks use a proportional-integral-derivative feedback system. Assume that the output from our FPI is some output that we label $Y(t)$. The PID 110 provides a stable reference point, or set point, $R(t)$. The error signal in the system will then be $e(t)=R(t)-Y(t)$. The PID 110 then acts on the error signal and produces a control output

$$
u(t)=K_{p} e(t)+K_{i} \int_{0}^{t} e(\tau) d \tau+K_{d} \frac{d e(t)}{d t}
$$




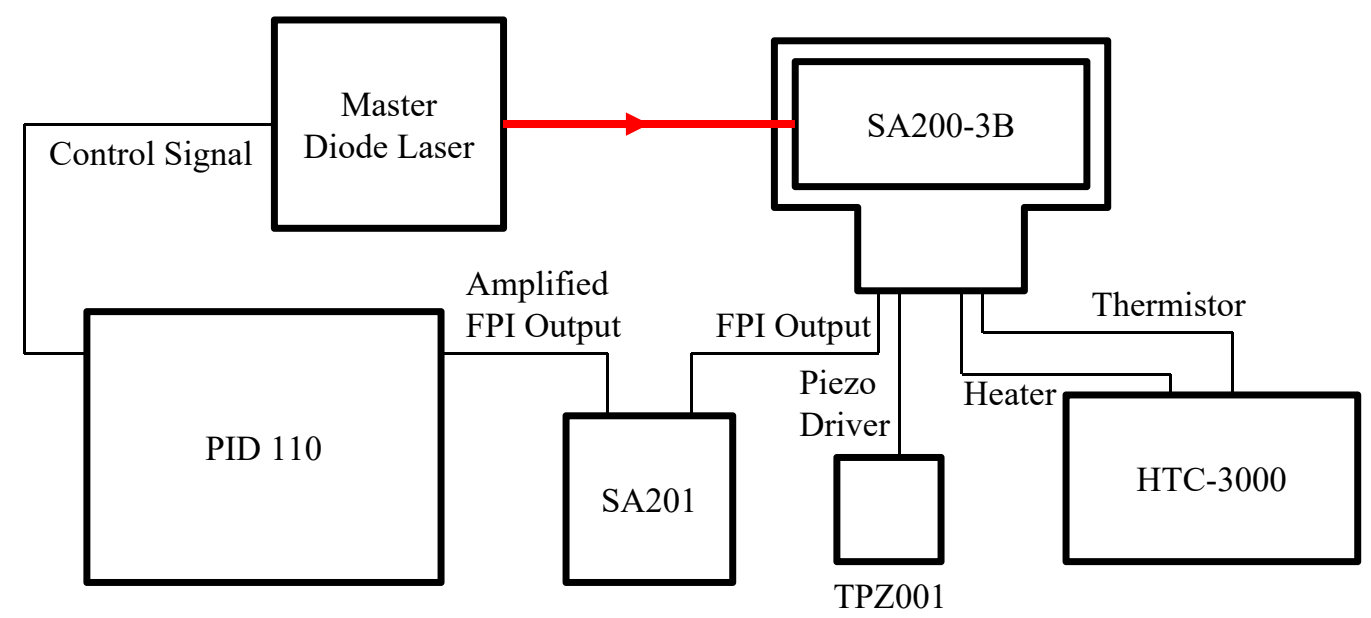

Figure 2.14: A schematic of the frequency locking system for the Toptica TA-SHG Pro. The red line is the infrared $(960 \mathrm{~nm}$ ) light going from the master diode laser to the Fabry-Pérot Interferometer (SA200-3B). The signal from the FPI goes to the amplifier (SA201) and from there goes to the feedback controller (PID 110). The control signal goes from the PID 110 to the master diode laser and adjusts the frequency. The piezo driver (TPZ001) sends a voltage to the FPI while the temperature controller (HTC-3000) sends a current to the heaters wrapped around the FPI and reads the temperature of the FPI using a thermistor. 
where $K_{p}, K_{i}$, and $K_{d}$ are the proportional, integral, and derivative constants. The first term in equation 2.14 is the proportional term, the second is the integral term, and the third is the derivative term [84]. The constants in equation 2.14 are tuned to give the highest frequency stability. Once locked, any changes in laser frequency will be measured by the PID, sent to the PID 110 as an error signal, and corrected. By adjusting the cavity length (via the piezoelectric driver), the frequency of the laser can be fine-tuned and swept.

To tune the settings of the PID 110, begin by setting the proportional, integral, and derivative constants to zero and the gain to $\sim 2$. With the lock switch set to "lock", increase the integral constant until the error signal begins to oscillate and then reduce the integral constant to approximately half of that value. Repeat this procedure for both the proportional and derivative constants. Typical values for these constants are 4 turns, 2.5 turns, and 1.5 turns of the trimpots that control the proportional, integral, and derivative constants, respectively.

To ensure the stability of the laser frequency, the FPI needs to be highly stable itself. The stability of the FPI is tied to one quantity that we can control: the optical path length. The cavity length, and thus the optical path length, is controlled by stabilizing the temperature of the cavity. Temperature control is achieved using heaters secured to the cavity and a PID feedback system provided by a Wavelength Electronics HTC-3000. This system allows us to get to within $0.02{ }^{\circ} \mathrm{C}$ of temperature stability. The temperature of the cavity is monitored using a thermistor that sends the temperature back to the HTC-3000 and to a National Instruments USB-6251 DAQ that allows us to monitor and record the temperature of the cavity. The optical path length is further controlled by controlling the pressure of the air within and around the cavity and achieved by putting the FPI inside a sealed can. 
(a)
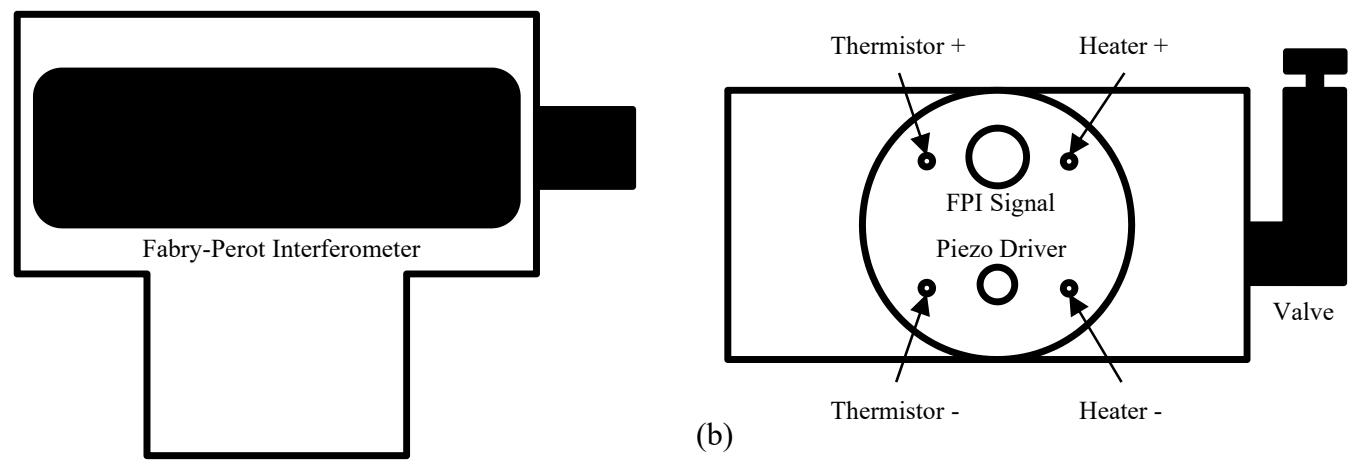

Figure 2.15: A schematic drawing of the "T" used for controlling the pressure of the FabryPérot Interferometer. (a) The top view shows the "T" shape with the FPI resting inside the main part of the "T". (b) The side view shows the electrical feedthroughs and the valve that is used for pressure tuning of the FPI frequency.

The sealed can consists of a vacuum "T" that has three ports (see Figure 2.15). The first port has a window to allow the infrared light of the laser to reach the FPI. The second port has all of the electrical feedthroughs that connect to the signal output, the piezoelectric input, the heaters, and the thermistor. The third port has a sealed valve that allows for pressure tuning of the optical path length, and thus the assumed frequency of the FPI, by changing the volume of the pressure can. We use pressure tuning to do fine adjustments of the laser frequency without disturbing the piezoelectric which has a long settling time. The pressure can is additionally wrapped in insulation and put within a foam box for further temperature isolation. The can also sits on a sorbothane pad to decouple it from vibrations on the table. 


\subsection{Software}

\subsubsection{Data Acquisition}

All of the data acquired in the experiments described below were taken using a LabView program originally written by Jon White and heavily modified by Tao Zhou and myself [5]. This program was originally used to read a field ionization trace from a Tektronix TDS 3054B oscilloscope, gate the signal, and integrate the signal. It has since been modified to allow for greater automation and control of the devices used in the experiments. The modifications to the program include control of:

- Two oscilloscopes, allowing for measurement of both the field ionization trace and the MOT fluorescence.

- The DG 645 delay generator, allowing for control of the delay length of a particular channel and to send trigger events when in single-shot mode.

- The AWG 510 to send event trigger pulses that begin the pulse sequence output from the AWG.

- The acusto-optic modulator, allowing for control of the AOM frequency.

- A delay stage (used in experiments by Tao Zhou and described in [45]), allowing for control of the stage's position and the start of the movement sequence for the stage.

- Sensors for monitoring the temperature inside of and voltage applied to the FabryPerot Interferometer. 
There are two different versions of the LabView program for different acquisition rates. One version runs at $15 \mathrm{~Hz}$ and is synchronized to the DG 645. The other version is a singleshot implementation that sends a user-defined trigger signal to the DG 645 that activates the delay sequence.

The output from the program has also been expanded to include the full field ionization trace from each shot in addition to the gated and integrated output. This allows for additional processing to be done to the raw data and for post-collection gating and integration. Output of the MOT fluorescence trace is also available and the timing of when those traces are taken is different for every experiment and will be described in those chapters.

\subsubsection{Analysis}

All data analysis presented in this dissertation was performed using the open-source statistical language R [85]. Additional packages used during data analysis include:

- dplyr [86], tidyr [87], readr [88], and tibble [89] for data processing and manipulation;

- ggplot2 [90] and gtable [91] for figure creation;

- caTools [92] and gsl [93] for additional functions and calculations; and

- signal [94] for digital signal processing and data filtering.

Calculations of the energy shift of ${ }^{85} \mathrm{Rb}$ atoms due to the Stark effect were done for several of the experiments and the techniques used can be found in the starkr package [95]. The starkr package contains functions for determining the quantum defect and radial matrix elements of ${ }^{85} \mathrm{Rb}$, computing Stark matrix elements, diagonalizing Stark matrices, building Stark 
energy maps, determining energy crossings of pair states, and determining the character of Stark eigenstates as a function of electric field. These functions were used throughout our calculations and will be further described in the following chapters. 


\section{Chapter 3}

\section{Absence of Collective Decay in a Cold Rydberg Gas}

This chapter describes an investigation of the decay of cold Rydberg atoms in a MOT. Of particular relevance to this dissertation is the work on the characterization of the conditions in the MOT and their influence on Rydberg decays. This characterization was critical to interpreting the experiments. The bulk of the measurements and calculations were performed by Tao Zhou and can be found in [45] and [6]. The experiment, results, and analysis are reproduced from [6] with some modifications and additions.

\subsection{Introduction}

Atoms within cold Rydberg ensembles are coupled by strong long-range dipole-dipole (DD) interactions [8], making them interesting systems for exploring few- and manybody quantum dynamics in general and applications in quantum information in particular 
$[12,14,18,19,21,25-28,30-35,37-39,42,43,96-109]$. Of course, unlike in ground-state systems, finite Rydberg lifetimes limit the types of measurements and number of coherent manipulations that can be performed in a given experiment. Fortunately, isolated Rydberg atoms exhibit low spontaneous decay rates [8], potentially enabling processing over microto millisecond time scales. At first glance this stability against radiative decay might seem surprising given the large transition matrix elements between adjacent Rydberg states, which scale as $n^{2}$. However, spontaneous decay to nearby levels via low-frequency emission is strongly suppressed by the $\omega^{3}$ dependence of the Einstein $A$ coefficient. As a result, the predominant decay path for isolated Rydberg atoms in low-angular-momentum states is to the ground or low-lying excited levels, resulting in a $n^{-3}$ scaling of the spontaneous emission rate [8].

That said, neighboring Rydberg levels can play a dominant role in the decay of a large number $N$ of atoms which are either simultaneously excited in a volume with dimensions smaller than the wavelength $\lambda$ of the emitted light, or sequentially excited throughout a cylindrical volume with length $L \gg \lambda[110,111]$. In his seminal paper [112], Dicke predicted that a dense collection of $N$ radiators, either in very close proximity or in a properly phased extended distribution, could develop spontaneous correlations and collectively emit radiation at rates greatly exceeding ("superradiance"), or much smaller than ("subradiance"), those of individuals in the sample. For the two-level systems considered by Dicke, correlations between a large number of emitting atoms can initiate collective superradiant emission at a per atom rate up to N/4 times larger than that between the same two levels in an isolated atom [112]. Collective emission remains a subject of considerable interest in many different contexts, including Rydberg atoms [44, 113-124]. 
The presence of blackbody radiation, the existence of multiple photodecay channels in a Rydberg ladder, and strong DD interactions between atoms, all explicitly neglected in Dicke's original paper (and in many subsequent treatments) $[112,125,126]$, make it much more difficult to observe, characterize, and quantitatively predict collective decay phenomena in Rydberg gases. In particular, DD interactions can suppress superradiance. In a thermal gas, this suppression results from DD-mediated collisions that homogeneously dephase the individual emitters in the ensemble at a rate greater than the superradiance rate [123]. In a frozen gas, DD exchange interactions couple pairs, or larger groups, of atoms leading to a variation in transition energies across the ensemble. Such inhomogeneities squelch the correlations that underlie superradiance, along with the collective emission [120]. That said, a clear signature of superradiance between Rydberg states, a fluorescence cascade from a Rydberg ladder proceeding at a rate much greater than spontaneous emission of isolated atoms, was first observed following pulsed-laser excitation of an elongated volume $(L \gg \lambda)$ in a thermal cell [110]. More recently, direct evidence for superradiance was found in the millimeter-wave emission from a large cylindrical volume of Ca Rydberg atoms in a supersonic expansion, also with $L \gg \lambda[122,123]$. As pointed out in the latter work, the rates for superradiant decay and DD dephasing within a given decay channel are essentially identical, up to a multiplicative geometric factor $L / \lambda$ in the superradiance rate formula. Accordingly, it was suggested that collective decay should not play a major role in Rydberg depopulation unless $L \gg \lambda[123]$.

Still, despite competing DD effects, under certain conditions superradiance should play some role in cold ensembles where the dimensions of the excited volume are less than or comparable to $\lambda$. Indeed, evidence of reduced Rydberg lifetimes has been reported 
in several such experiments [44, 121, 124, 127, 128]. For example, Feng et al. observed a density-dependent lifetime suppression of Cs Rydberg atoms in a magneto-optical trap (MOT) [127]. They attribute the suppression to a combination of neutral Rydberg atom collisions and superradiance. However, the evidence for superradiance appears tenuous as their calculations with and without superradiant contributions both fall within their measurement uncertainty (see their Fig. 2). In addition, if one applies their values for Rydberg collision velocity and cross section, the collisional depopulation rates are over three orders of magnitude too small to account for their observations. Han and Maeda attributed population transfer from initial to neighboring Rb Rydberg state to superradiance, but provided no evidence ruling out other possible population transfer mechanisms [129]. In other measurements, using fluorescence detection, Day et al. found Rydberg depopulation rates that were roughly twice that expected from single-atom spontaneous emission over a range of $n$ states and at low densities, $\rho \sim 1 \times 10^{7} \mathrm{~cm}^{-3}$ [121]. The small variation of the lifetime suppression with principal quantum number couple with trap loss measurements argued against collisional depopulation and blackbody ionization. Instead, the enhanced Rydberg decay rate was found to be qualitatively consistent with a simplified collective decay model. In other experiments, the inclusion of superradiant decay channels was found to improve the quality of model fits to electromagnetically induced transparency measurements in cold Rydberg gases [124,128].

Certainly, a substantial decrease in Rydberg lifetimes due to collective emission would have a significant impact on most cold Rydberg atom experiments. Perhaps more important, in the context of the exploration and control of few- or many-body Rydberg dynamics, are the influences of the spontaneous quantum correlations that are predicted to develop with 
the emission of the first photon from the sample and evolve as the Rydberg population descends through a ladder of Dicke states $[112,126]$. Interestingly, Wang et al. presented a sophisticated theoretical treatment of photodecay in a multilevel Rydberg system which makes definite predictions as to whether superradiance should occur for a given initial principal quantum number, atom density, and experimental volume [44]. The theory apparently reproduced the rapid decay, at a rate approximately 40 times greater than predicted from spontaneous emission alone, of an initial population of 43p atoms in a MOT at a density of $\rho \sim 5 \times 10^{8} \mathrm{~cm}^{-3}$.

We have used pulsed-laser excitation of Rb Rydberg atoms in a MOT under conditions ostensibly similar to those used in Ref. [44] in an attempt to test the predictions of their Rydberg superradiance theory. We employ state-selective field ionization (SSFI) to measure the population in the initial and neighboring Rydberg states as a function of delay after the laser excitation. We find no evidence for the predicted collective decay over a range of principal quantum numbers $26 \leq n \leq 40$ and atom densities $\rho \sim 3 \times 10^{9} \mathrm{~cm}^{-3}$, despite the fact that, for these states, our highest density is more than two orders of magnitude above the predicted superradiance threshold [44]. Instead, our measurements are consistent with noncorrelated spontaneous decay combined with population redistribution via blackbody radiation.

\subsection{Experimental Procedure and Results}

In these experiments, ${ }^{85} \mathrm{Rb}$ atoms at $70 \mu \mathrm{K}$ are held in a MOT (described in Section 2.2). The MOT is positioned at the center of four parallel rods which facilitate the application of 
static and pulsed electric fields in the $y$ direction for exciting and detecting Rydberg atoms in the MOT. A $10 \mathrm{~ns}$ pulsed, tunable, dye-amplified, $\sim 480 \mathrm{~nm}$ diode laser (described in Sections 2.1.1 and 2.1.3) propagating in the $x$ direction is focused into the center of the MOT, creating a cylindrically shaped volume of cold Rydberg atoms with a full width at half maximum (FWHM) diameter of $\sim 0.1 \mathrm{~mm}$ and a length of $0.4 \mathrm{~mm}$. The MOT and Rydberg lasers are non collinear, preventing the excitation of Rydberg atoms throughout any extended volume from the lower-density background of thermal $\mathrm{Rb}$ atoms in the chamber. The $\sim 150 \mathrm{MHz}$ bandwidth of the Rydberg excitation laser ensures that there is no excitation suppression via dipole blockade $[12,14]$. At a tunable time $\tau$ after the laser excitation, a ramped voltage is applied to two of the rods, ionizing any Rydberg atoms in the interaction region and pushing the resulting ions toward a microchannel plate (MCP) detector. Ions originating from different Rydberg states arrive at the detector at different times. The integrated signals in different time bins are proportional to the populations in different Rydberg states and are recorded for each laser shot as a function of the ionization time $\tau$. The experiment proceeds at the $15 \mathrm{~Hz}$ dye-laser repetition rate. Further details on the electronics used in this experiment can be found in Section 2.3.

The diode laser is tuned to selectively excite atoms from the upper $5 p$ trap level to $n s$ or $n p$ Rydberg states with $26 \leq n \leq 46$. Excitation of $n p$ states is facilitated by the application of a weak static electric field (from $30 \mathrm{~V} / \mathrm{cm}$ at $n=26,16 \mathrm{~V} / \mathrm{cm}$ at $n=32$, to $7 \mathrm{~V} / \mathrm{cm}$ at $n$ $=40$ ). The density of $5 p$ atoms in the MOT is determined, to within $30 \%$, by combining measurements of the spatial dimensions of the atom cloud size via direct imaging with a CCD camera with measurements of the radiated fluorescence using an optical power meter. By saturating the Rydberg excitation using high laser fluence, we ensure that $50 \%$ of the $5 p$ 
atoms within the interaction volume are excited to Rydberg states, enabling us to determine the Rydberg atom density (for more details on this method see Section 2.2.3). Subsidiary experiments on resonant energy transfer between Rydberg atoms are consistent with the Rydberg density determination (see $[5,130]$ and Chapter 4). The MOT fluorescence is monitored throughout the lifetime measurements, ensuring that the number of atoms in the MOT is constant to within a few percent as $\tau$ is scanned. Care is taken to minimize the amplified spontaneous emission (ASE) from the dye-amplified laser pulse, eliminating direct photoionization of $5 p$ atoms. This is done by rotating the UV pump light so that any ASE will not be perfectly collinear with the seed light. For the $s$-state measurements, a small, $\sim 1.4 \mathrm{~V} / \mathrm{cm}$, residual electric field persists in the interaction region due to imperfect shielding of the high-voltage-biased MCP (a larger field is present for initial $p$ states). This field is sufficient to eject any ions or electrons from the interaction region, eliminating extended interactions between charged particles and neutral Rydberg atoms, and preventing the spontaneous evolution of the Rydberg gas into a plasma [131,132]. Neither the small static field employed for the $p$-state measurements nor the smaller residual field present during the $s$-state measurements substantially alters the rates for spontaneous emission or population transfer induced by blackbody radiation. The potential influence of the field inhomogeneity on superradiant decay is considered in Sections 3.3 and 3.4.

Figures 3.1 and 3.2 show our principal experimental results. In Figs. 3.1(a) and 3.1(c), the probabilities for finding atoms in the $26 s+25 p, 32 s$, and $40 s$ states are plotted as functions of detection time $\tau$ for the maximum densities explored, $\rho \sim 3 \times 10^{9} \mathrm{~cm}^{-3}$ and $\rho \sim 1.5 \times 10^{9} \mathrm{~cm}^{-3}$, respectively. Note that for the lowest initial $n$ state the sum of the $26 \mathrm{~s}$ and $25 p$ populations is shown since their corresponding features could not be adequately 

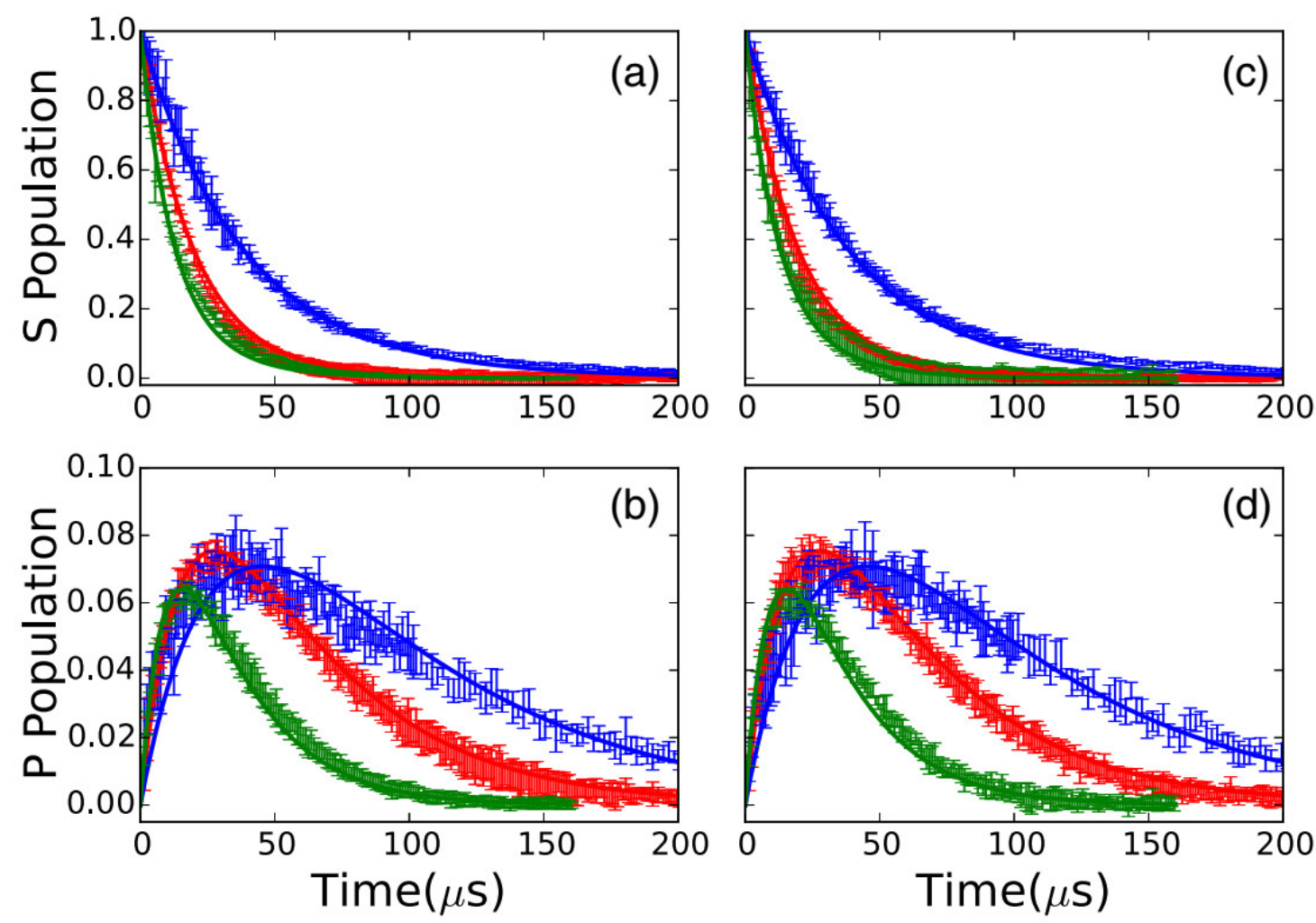

Figure 3.1: (a),(c) Probabilities for finding atoms in $26 s+25 p$ (green, fastest decay), $32 s$ (red, intermediate decay), and $40 \mathrm{~s}$ (blue, slowest decay) as a function of detection time $\tau$ for Rydberg densities of $\rho \sim 3 \times 10^{9} \mathrm{~cm}^{-3}$ (a, b) and $\rho \sim 1.5 \times 10^{9} \mathrm{~cm}^{-3}$ (c, d). Note that the sum of the $26 s$ and $25 p$ populations is shown since their corresponding features could not be adequately separated in the field-ionization signal. Vertical bars show the experimental data with uncertainties, and the solid curves are calculated as described in the text. Measurements and calculations for the $40 \mathrm{~s}$ decay extend to $500 \mu \mathrm{s}$ where the remaining population is negligible. (b),(d) Probabilities for finding the atoms in $26 p$ (green, fastest rise and decay), $32 p$ (red, intermediate rise and decay), and $40 p$ (blue, slowest rise and decay) levels as a function of detection time $\tau$. The states are populated by blackbody redistribution from the initial $26 s, 32 s$, and $40 s$ levels, respectively. The data was measured simultaneously with those shown in (a) and (c). Vertical bars show the experimental data with uncertainties and the solid curves are calculated as described in the text. The measured $p$-state probabilities are normalized to the calculations as described in the text. The calculations have no free parameters and consider only the effects of spontaneous emission and blackbody radiation on isolated atoms. 

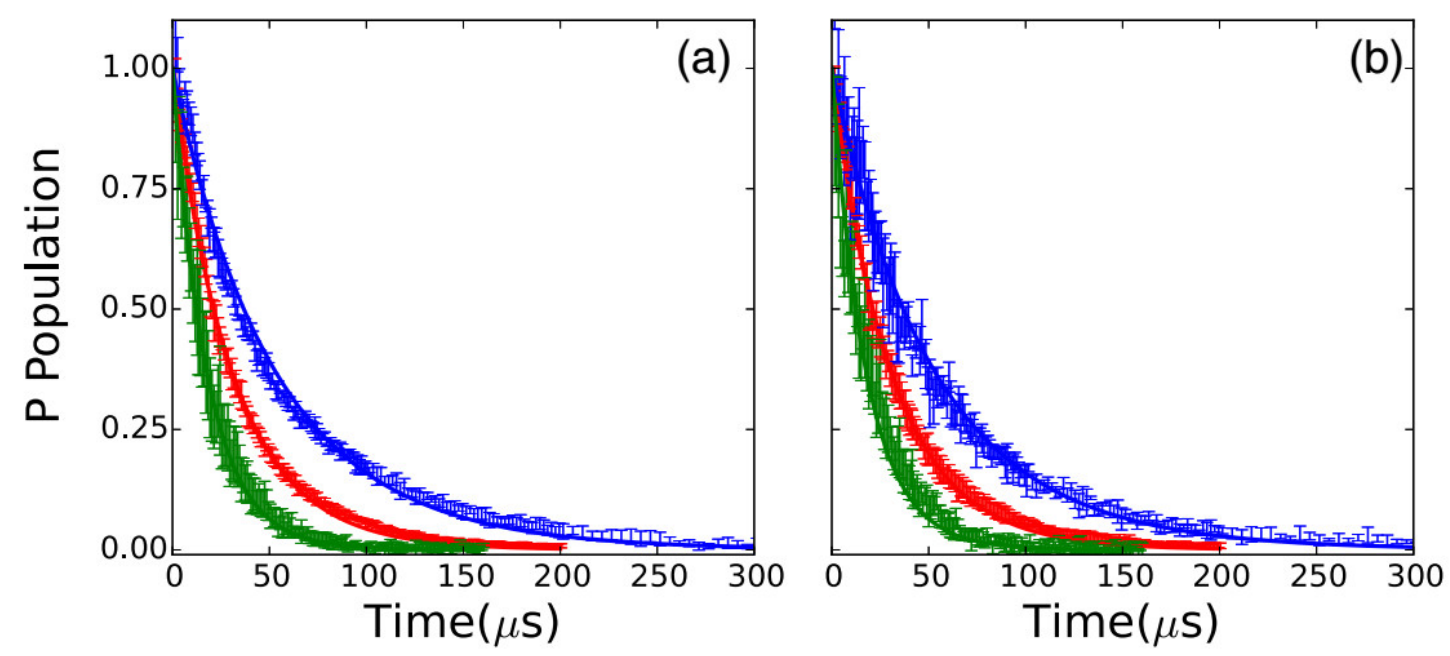

Figure 3.2: Probabilities for finding atoms in $26 p$ (green, fastest decay), $32 p$ (red, intermediate decay), and $40 p$ (blue, slowest decay) as functions of detection time $\tau$ for Rydberg densities of $\rho \sim 3 \times 10^{9} \mathrm{~cm}^{-3}$ (a) and $\rho \sim 1.5 \times 10^{9} \mathrm{~cm}^{-3}$ (b). Vertical bars show the experimental data with uncertainties and the solid curves are calculated as described in the text. Measurements and calculations for the $40 p$ decay extend to $500 \mu \mathrm{s}$ where the remaining population is negligible. The calculations have no free parameters and consider only the effects of spontaneous emission and blackbody radiation on isolated atoms. 
separated in the time-resolved field-ionization signal. Within experimental uncertainties, the decays for the three initial $s$ states are identical at the two densities shown. Additional measurements were made at Rydberg densities as low as $\rho \sim 2 \times 10^{8} \mathrm{~cm}^{-3}$ (for $32 \mathrm{~s}$ initial states) and $\rho \sim 5 \times 10^{8} \mathrm{~cm}^{-3}$ (for $32 p$ initial states), but no statistically significant differences were observed in the decays.

For spontaneous decay of isolated atoms at absolute zero, one would expect lifetimes of 28 and $58 \mu$ s for the $32 s$ and $40 s$ atoms, respectively [110]. The measured lifetimes for the $32 s$ and $40 s$ are substantially smaller, 19 and $38 \mu$ s, respectively, due to population redistribution by blackbody radiation from the $300 \mathrm{~K}$ environment surrounding the MOT. Indeed, redistributed population is detected in neighboring Rydberg levels. In particular, Figs. 3.1 (b) and 3.1 (d) show the delay-dependent population in the $p$ states $(26 p, 32 p$, and $40 p$ ) that lie immediately above the respective initial $s$ states. Although we would expect to find some atoms in the adjacent, lower-lying $p$ states as well, small features reflecting that population in the time-of-ionization signal lie within the initial-state peak (for the case of $26 s$ ) or are masked by the tail of the larger, initial-state peak which precedes it.

The measured lifetime for the combined $26 s+25 p$ states is $14 \mu \mathrm{s}$. The same as that expected for spontaneous decay of the $26 s$ alone [110]. Simulations (described in detail in Section 3.3) indicate that this apparent agreement is not due to the absence of blackbody transfer out of $26 s$. Rather, the small longer-lived $25 p$ component of the signal masks much of the change in the $26 s$ decay, with a predicted effective lifetime of $13 \mu$ s for the $26 s+25 p$ combination, similar to what we observe. We note that due to the slew rate of the ionizing field, there is a distribution of ionization times and, therefore, of detection efficiencies for atoms in different states. As a result, each of the measured $p$-state populations in Figs. 
3.1(b) and 3.1(d) has been multiplied by a normalization factor to obtain the best agreement with the calculated decay curves that are shown in the figures and described in Section 3.3.

Figure 3.2 shows analogous data for the decay of initially excited $26 p, 32 p$, and $40 p$ states. Again, due to blackbody redistribution, the lifetimes associated with these decays $(18,31$, and $51 \mu \mathrm{s})$ are considerably smaller than expected from spontaneous emission alone (37, 75, and $155 \mu \mathrm{s})$ [110]. However, in this case, no substantial population is detected in the neighboring $s$ or $d$ levels. The analysis described in the next section indicates that the populations in these states are not detectable within our signal-to-noise ratio, remaining at or below the few percent level due to the relatively rapid spontaneous emission rate out of the $s$ states, and smaller $p \rightarrow s$ and $p \rightarrow d$ blackbody transition rates.

\subsection{Analysis}

To determine if collective processes play any significant role in the decays we observe, we compare the measurements to the results of a simple rate equation model. The model includes population transfer via stimulated emission and absorption of blackbody radiation between an essential set of $s, p$, and $d$ Rydberg states neighboring the initial level, as well as spontaneous emission out of those essential states to (undetected) lower-lying levels. We calculate the blackbody transition rates between the essential states [8] as well as the known total spontaneous emission rates of the $s, p$, and $d$ Rydberg levels [8].

For example, for an initially excited $40 \mathrm{~s}$ state, the rate equation describing the time- 
dependent population in the initial $40 \mathrm{~s}$ level is

$$
\frac{d N_{40 s}}{d t}=\left(-A_{40 s}-B_{40 s \rightarrow 40 p}-B_{40 s \rightarrow 39 p}\right) N_{40 s}+B_{39 p \rightarrow 40 s} N_{39 p}+B_{40 p \rightarrow 40 s} N_{40 p}
$$

where $N_{n \ell}$ is the population in state $n \ell, A_{40 s}$ is the $40 \mathrm{~s}$ spontaneous decay rate and $B_{n \ell \rightarrow n^{\prime} \ell^{\prime}}$ is the blackbody transition rate from $n \ell$ to $n^{\prime} \ell^{\prime}[8]$ :

$$
B_{n \ell \rightarrow n^{\prime} \ell^{\prime}}=2 \bar{n} \alpha^{3} \omega_{n \ell, n^{\prime} \ell^{\prime}}^{2}\left|\bar{f}_{n \ell, n^{\prime} \ell^{\prime}}\right|
$$

In Eq. 3.2, $\bar{n}=\left(e^{\omega_{n \ell, n^{\prime} \ell^{\prime}} / k T}-1\right)^{-1}$ is the photon occupation number at the frequency $\omega_{n \ell, n^{\prime} \ell^{\prime}}$ corresponding to the energy splitting between states $n \ell$ and $n^{\prime} \ell^{\prime}, k$ is Boltzmann's constant, $T$ is the temperature, $\alpha$ is the fine-structure constant, and $\left|\bar{f}_{n \ell, n^{\prime} \ell^{\prime}}\right|$ is the magnitude of the oscillator strength averaged over all orientations of the initial and final states $n \ell$ and $n^{\prime} \ell^{\prime}$. We use the spontaneous emission rates calculated by Gounand [110].

The populations in the secondary states $39 p$ and $40 p$ are computed using similar rate equations that include the total spontaneous decay rate out of those levels as well as blackbody transitions to and from pairs of $s$ and $d$ levels that lie immediately above and below each $p$ state. We truncate the system of equations with rate equations that include spontaneous decay from the tertiary $s$ and $d$ levels and their blackbody couplings with the secondary states. Analogous systems of equations are used to compute the Rydberg population decay following initial $p$-state excitation. We note that, for initial or intermediate $p$ states in particular, blackbody radiation redistributes a small, but non-negligible, fraction of the initial population beyond the nearest-neighbor $s$ and $d$ states. Therefore, an approximate 
expression [8]

$$
B_{n \ell}=\frac{4 \alpha^{3} k T}{3 n^{2}}
$$

for the total blackbody decay rate from each $p$ level is used to more accurately account for the net transfer out of these states.

The results of our calculation, which ignore any collective decay phenomena are shown with the data in Figs. 3.1 and 3.2. Overall, the agreement is reasonable. Aside from the previously noted renormalization of the experimental $p$-state population, no parameter adjustments have been made to obtain the level of agreement shown. The data provide no evidence of a significant reduction in the Rydberg lifetimes due to superradiance. This is true over a range of principal quantum numbers and atom densities where superradiant emission has been predicted to be the dominant decay path [44].

It is well established that superradiance is suppressed by inhomogeneities in transition energies across a sample of emitters [120], and we suspect that this is the case in our, and many other, cold atom experiments. In our experiments, three different effects contribute to such inhomogeneities. The first, and dominant mechanism for some of our measurements, is the DD exchange interaction. Consider a pair of identical atoms with two levels $s$ and $p$ and interatomic separation $R$. Spontaneous emission from the initial upper pair state $s s$ results in the population of the bright configuration of the lower-energy pair state $(s p+p s) / \sqrt{2}$. However, due to the DD coupling between the atoms, $V_{D D} \propto|\langle s|r| p\rangle|^{2} / R^{3}$, the energy of this state is not the same as that for two atoms at infinite separation [8]. Accordingly, in a large ensemble of randomly spaced atoms, every possible configuration of $N_{s} s$ atoms and $N_{p} p$ atoms has a different energy, depending on the separation (and relative orientation) between the $p$ atoms and their neighboring $s$ atoms. As a result, any Dicke state, the bright 
linear combination of all possible configurations of $N_{s} s$ atoms and $N_{p} p$ atoms [112], is nonstationary. The phases of the constituent $N$-atom product states evolve at different rates, as determined by their DD energy shifts relative to their energies at infinite separation. The emission from these nonstationary Dicke states dephases at a rate comparable to the typical dipole-dipole energy shift $\bar{V}_{D D}$ for pairs of atoms in the ensemble. Superradiance cannot occur unless the system transitions down each step in the Dicke ladder more rapidly than this dephasing. A similar argument has been made by Gross and Haroche [133]. In the frequency domain, atoms with different transition energies at different locations in the ensemble do not collectively emit into the same field unless that emission occurs in a very short burst with a sufficiently broad, coherent bandwidth.

To determine the DD dephasing rate, we use the most probable nearest-neighbor separation in a random ensemble, $R \simeq(2 \pi \rho)^{-1 / 3}$, and average over all orientations of the Rydberg states on any two neighboring atoms $n s$ and $n^{\prime} p$ to obtain $[36,134]$

$$
\bar{V}_{D D}=\frac{8 \pi}{9} \rho\left|\left\langle n s|r| n^{\prime} p\right\rangle\right|^{2}
$$

Using a numerical Numerov integration algorithm to compute the relevant radial matrix elements [135], at the highest density studied $\left(\rho=3 \times 10^{9} \mathrm{~cm}^{-3}\right)$ we obtain values for the DD exchange coupling between the $n s$ and $(n-1) p$ states, $\bar{V}_{D D}=2.4,6.2$, and $17 \mathrm{MHz}$, for $n=26,32$, and 40, respectively. These interaction strengths set effective lower limits for the rates at which collective emission from $n s$ to $(n-1) p$ can occur. Similarly, for initial $n p$ states and $\rho=3 \times 10^{9} \mathrm{~cm}^{-3}$, the relevant exchange coupling is to the nearest lower-lying $s$ states with $\bar{V}_{D D}=3.1,7.8$, and $20 \mathrm{MHz}$, for $n=26,32$, and 40, respectively. The magnetic field gradient in the MOT is another source of energy inhomogeneities 
in our ensemble. As in Ref. [44], the magnetic field remains on during our measurements, resulting in a transition energy variation of approximately $1 \mathrm{MHz}$ across the MOT. This inhomogeneitiy is smaller, or much smaller, than that due to dipole-dipole interactions at sufficiently high densities. It should not play a principal role in suppressing superradiance under the conditions used to produce Figs. 3.1 and 3.2.

\subsection{Electric Field Characterization}

The third contributor to the Rydberg energy variations across the ensemble is electric field inhomogeneity. While the voltages applied to the field rods produce a field that is quite uniform over the MOT (predicted field variations of $0.47 \%$, corresponding to 141 $\mathrm{mV} / \mathrm{cm}$ for the largest applied field of $30 \mathrm{~V} / \mathrm{cm}$ for the $26 p$ measurements) the residual field from the MCP is not as uniform. Using a combination of spectroscopic measurements and accurate Stark energy calculations, we determine an upper limit for the Rydberg energy inhomogeneity due to the nonuniformity of the electric field $F$ in the interaction region.

First, we measure the transition frequencies for excitation of $32 p_{3 / 2}\left|m_{j}\right|=1 / 2,3 / 2$ from the $5 p_{3 / 2}$ upper trap state as a function of the voltage applied to the field rods (see Fig. 3.3). For convenience, in the following discussion we refer to the field produced by the rods as the "applied" field. The experimental geometry is identical to that used for the lifetime measurements (see Section 3.2), but the Rydberg excitation is performed with an unamplified, $3 \mu$ s pulse chopped from the $\sim 1 \mathrm{MHz}$ bandwidth $\mathrm{cw}$ diode laser. The Rydberg excitation pulse has $\sim 1 \mu$ s rise and fall times and is formed using an acousto-optic modulator (see description in Section 2.1.1). We use a temperature- and pressure-stabilized 
Fabry-Pérot interferometer (FPI) to track the relative frequency of the Rydberg laser as it is scanned (see Section 2.3.5). The laser frequency is scanned by applying a triangle wave to the piezoelectric element within the FPI to change the cavity length of the FPI. While locked, the changing cavity length leads to a change in the laser frequency. We obtain a calibration for the laser frequency vs the voltage applied to the FPI by applying an AC electric field of known frequency $(\sim 12 \mathrm{MHz})$ on a few sample scans, creating a sideband at the applied frequency. The population in $\left|m_{j}\right|=1 / 2$ is distinguished from that in $\left|m_{j}\right|=3 / 2$ using SSFI. By recording the signal in two different time bins we obtain (nominally) separate excitation profiles to the two $\left|m_{j}\right|$ states in the same laser frequency scan. Therefore, the energy splitting between the two $\left|m_{j}\right|$ states can be accurately determined to well within the excitation bandwidth which is dominated by the $6.07 \mathrm{MHz}$ natural linewidth of the initial $5 p_{3 / 2}$ level.

In zero electric field, the excitation profiles associated with the population in the two $\left|m_{j}\right|$ levels should exhibit maxima at the same laser frequency, i.e., have zero energy splitting. However, as shown in Fig. 3.4, we observe a minimum splitting of $2 \mathrm{MHz}$ at an applied field of $-2.758 \pm 0.005 \mathrm{~V} / \mathrm{cm}$. The minimum splitting at a nonzero applied field allows us to determine the components of the MCP field parallel and perpendicular to the applied field. Apparently, the application of a $-2.758 \mathrm{~V} / \mathrm{cm}$ rod field minimizes the net field in the interaction region. Accordingly, there must be a parallel, $2.758 \mathrm{~V} / \mathrm{cm}, \mathrm{MCP}$ field component which we call the "offset" field. Using the variation in the $\left|m_{j}\right|$ splitting as a function of applied field, we can also extract a value, $1.38 \pm 0.04 \mathrm{~V} / \mathrm{cm}$, for the perpendicular, i.e., "residual", MCP field component. The solid curve shown with the data in Fig. 3.4 is the predicted $32 p_{3 / 2}\left|m_{j}\right|=1 / 2,3 / 2$ splitting as a function of applied field (extracted from 


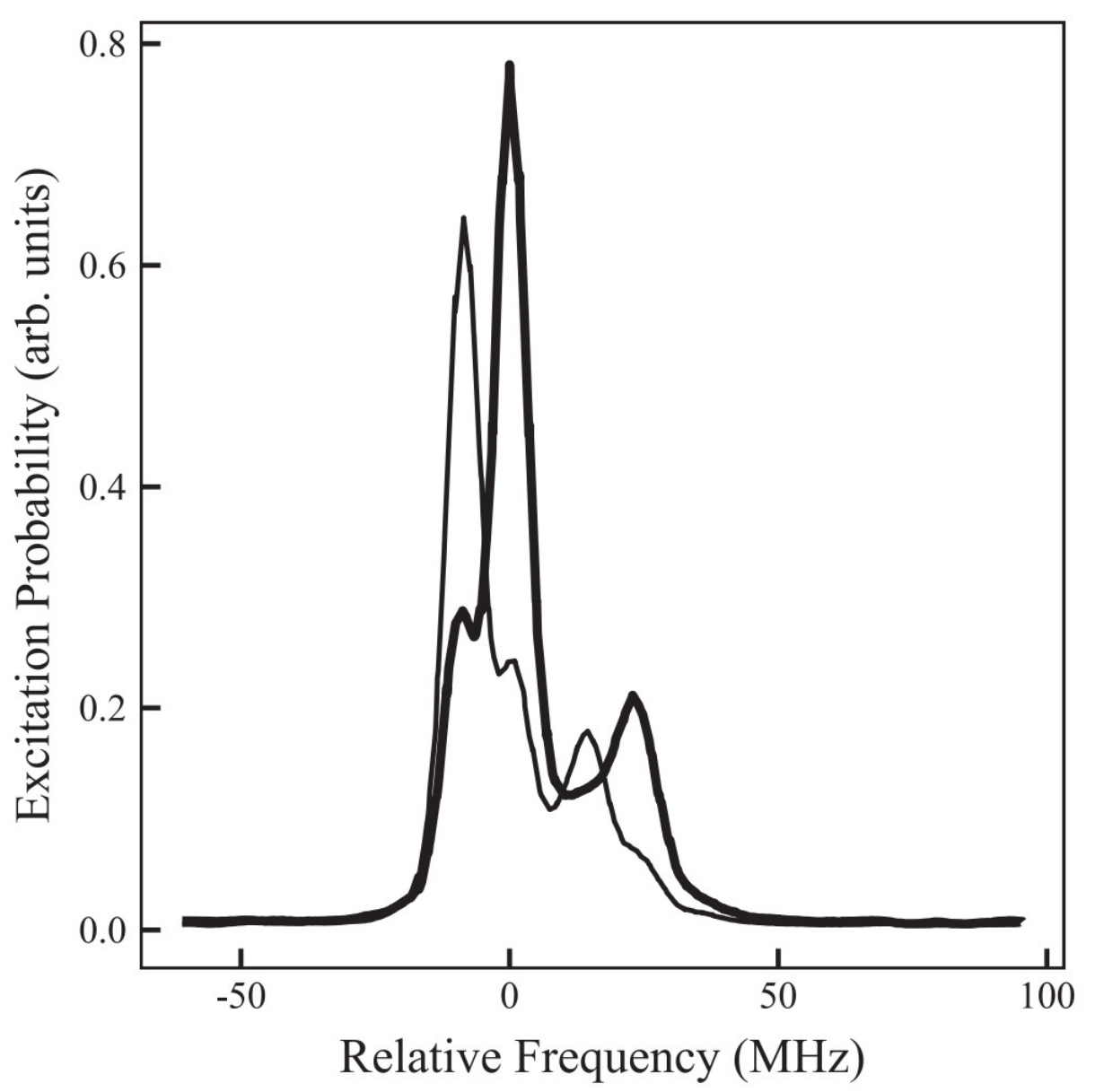

Figure 3.3: Measured 32p $p_{3 / 2}\left|m_{j}\right|=1 / 2$ (bold line) and $\left|m_{j}\right|=3 / 2$ (thin line) excitation probabilities as a function of Rydberg laser frequency in zero applied field. The two data curves are obtained simultaneously in the same laser frequency scan. The small feature on the left (right) of the main $\left|m_{j}\right|=1 / 2(3 / 2)$ peak is the result of imperfect discrimination of the $\left|m_{j}\right|=1 / 2$ and $3 / 2$ components via SSFI. The additional peak on the right of the main feature in each trace is due to the trap-laser dressing of the $5 p_{3 / 2}$ and $5 s$ levels. Its frequency shift from the main peak reflects the Autler-Townes splitting of the $5 p_{3 / 2}$ initial state. See Section 2.2.3 for more information on the Autler-Townes splitting. 
a full numerical Stark map calculation based on the method of Zimmerman et al. [135] and detailed in Section 2.4.2), assuming MCP offset and residual fields of $2.758 \mathrm{~V} / \mathrm{cm}$ and $1.38 \mathrm{~V} / \mathrm{cm}$, respectively. The Stark map produces the energy shift for a set of states as a function of electric field. By looking at the difference between the energy shifts of the two $\left|m_{j}\right|$ states, we obtain a good agreement with the experiment, confirming the accuracy of the calculation as well as the offset and residual field determinations. No free (fitting) parameters were used to match the Stark map calculation to the data outside of the -2.758 $\mathrm{V} / \mathrm{cm}$ offset applied to the field for the splitting and a $1.38 \mathrm{~V} / \mathrm{cm}$ field added in quadrature to the field during the Stark map calculation itself.

At, and near, the minimum splitting (i.e., in the presence of the residual field alone where the $s$-state decay measurements are performed), the $\left|m_{j}\right|$ excitation resonances have minimum linewidths of $8 \mathrm{MHz}$ (see Fig. 3.3). As noted above, the predominant contribution to this linewidth is the $6.07 \mathrm{MHz}$ natural linewidth of the $5 p_{3 / 2}$ level. However, the laser bandwidth, Zeeman shifts due to magnetic field inhomogeneities, and Stark shifts due to inhomogeneities in the $1.38 \mathrm{~V} / \mathrm{cm}$ residual field also contribute. Assuming that the laser spectrum and field distributions are Gaussian, the primary line shape is a Voigt profile whose FWHM can be approximated by [5]

$$
\Delta E=\frac{\Delta E_{H}}{2}+\sqrt{\frac{\Delta E_{H}^{2}}{4}+\Delta E_{i}^{2}}
$$

where $\Delta E_{H}$ is the homogeneous width and $\Delta E_{i}$ is the inhomogeneous width. Using Eq. 3.5, we extract a bandwidth of 3.9 MHz for the total Gaussian contribution. Accordingly, we obtain an upper-limit estimate for the electric field inhomogeneity by assuming it is the sole contributor to this width. From the Stark shift calculation of the $32 p_{3 / 2}\left|m_{j}\right|=$ 


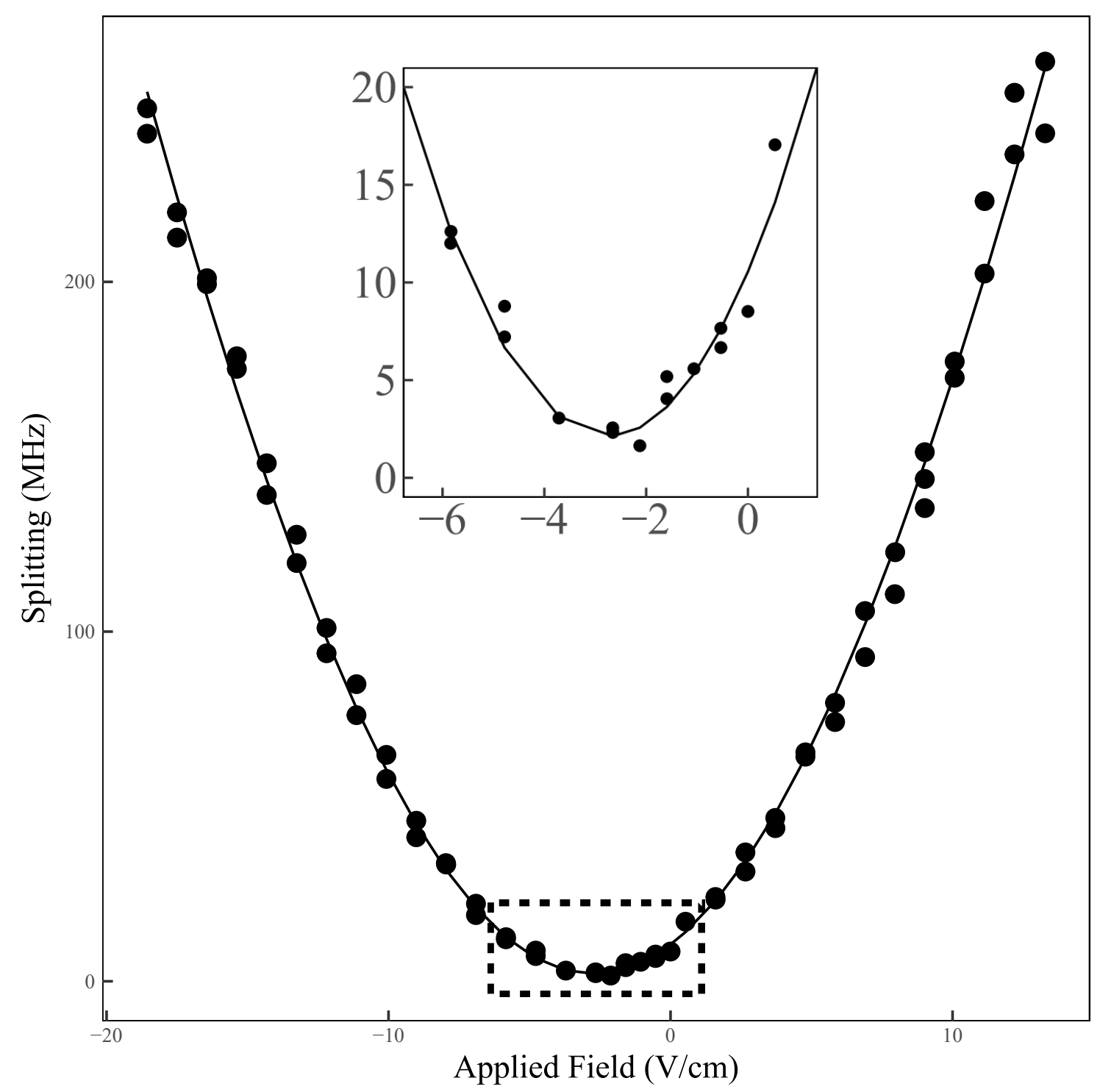

Figure 3.4: Difference (i.e. splitting) in the transition energies for exciting $32 p_{3 / 2}\left|m_{j}\right|=$ $1 / 2,3 / 2$ from $5 p_{3 / 2}$ as a function of applied electric field. Filled circles are measurements and the solid curve is the result of a numerical Stark map calculations assuming orthogonal "offset" and "residual" electric field components due to the MCP of $2.758 \mathrm{~V} / \mathrm{cm}$ and 1.38 $\mathrm{V} / \mathrm{cm}$, respectively. The inset shows a magnified view of the portion of the main figure within the dashed window. 
$1 / 2$ level, $\Delta E=\left[6.5 \mathrm{MHz} /(\mathrm{V} / \mathrm{cm})^{2}\right] F^{2}$, we determine that the maximum possible variation of the residual field across the interaction region is $\Delta F_{\text {res }}=0.20 \mathrm{~V} / \mathrm{cm}$. Using this field inhomogeneity with the field-dependent Stark shifts of the respective levels, we can compute the maximum range of transition energies between the initial $s$ states and the $p$ states immediately below them (to which the dipole coupling is the strongest). For the $26 s \rightarrow 25 p, 32 s \rightarrow 31 p$, and $40 s \rightarrow 39 p$ transitions, the maximum energy variations across the excitation region (with only the residual field present) are $0.45,2.2$, and $12 \mathrm{MHz}$, respectively. The transition energy variations are smaller for transitions to lower lying $p$ states due to the $n^{7}$ scaling of the Rydberg polarizability. So, at the highest densities we have explored, the energy inhomogeneities associated with the residual electric field are less, or much less, than those associated with the dipole-dipole exchange interaction. Therefore, the electric field inhomogeneities do not hold the primary responsibility for the suppression of superradiance from any of the initial $s$ states.

The situation with the initial $p$ states is somewhat different, as they are excited in a nonzero applied field that is considerably larger than the orthogonal residual field. As a result, the residual field and its inhomogeneity have essentially no effect on the transition energies. However, the spatial variations in the MCP offset field, which is parallel to the applied field, cannot be neglected. We use measurements of DD-mediated resonant energy transfer between Rydberg atoms to obtain an upper-limit estimate for the offset field inhomogeneity. Those experiments use the same experimental geometry as the Rydberg decay measurements and one (Ref. [130]) is detailed in Chapter 4 [36,130]. In the experiments, the probability for resonant population transfer from one pair of Rydberg states to another (e.g., $25 s+33 s \rightarrow 24 p+34 p$ [36]) is recorded as a function of an applied field which 
Stark-tunes the total energies of the atom pair in the two different configurations. In a uniform field, the line shape describing the field-dependent energy transfer probability is characterized by a peak at the "resonance" condition, where the total energies of the two sets of atom pair states are identical, and a width that is proportional to the Rydberg density. In a nonuniform field, the line shape has a nonzero minimum width as the density approaches zero, due to variations in the local field at different locations within the sample. Consider the $25 s+33 s \rightarrow 24 p+34 p$ resonance [36] for which maxima population transfer occurs in an electric field of $F \sim 3.4 \mathrm{~V} / \mathrm{cm}$. Assuming that the nonzero resonance width that is observed at very low Rydberg density [36] is due solely to the inhomogeneity in the electric field (i.e., ignoring magnetic field inhomogeneities and any other broadening effects) we obtain the maximum possible variation in the offset field, $\Delta F_{\text {off }}=0.08 \mathrm{~V} / \mathrm{cm}$, across the Rydberg sample. As an additional check, we consider a different energy transfer resonance, $32 p+32 p \rightarrow 33 s+32 s$, that is centered at a substantially higher field $F \sim 11.5 \mathrm{~V} / \mathrm{cm}[130]$. The nonzero low-density width for this energy transfer resonance gives the same maximum value for the offset field inhomogeneity, $\Delta F_{\text {off }}=0.08 \mathrm{~V} / \mathrm{cm}$.

Given $\Delta F_{o f f}$, we can compute the maximum possible variations in the energies, associated with transitions between initial $p$ states and the nearest lower-lying $s$ state, due to the inhomogeneous field. Using $\Delta F_{o f f}$, the calculated Stark shifts of each of the states involved in the transitions $26 p \rightarrow 26 s, 32 p \rightarrow 32 s$, and $40 p \rightarrow 40 s$, and the applied fields employed for the respective $p$-state excitations, we obtain the maximum possible transition energy variations due to the inhomogeneous electric field. These are 4.9, 13, and $30 \mathrm{MHz}$ for the $26 p, 32 p$, and $40 p$ initial states, respectively. Accordingly, for the $p$-state decays, the maximum energy variations due to the field are comparable to, but up to a factor of 
$1.7 \times$ larger than, those due to dipole-dipole interactions. Given our likely overestimate of the field inhomogeneity, both may play a role in suppressing collective emission from the ensemble.

\subsection{Conclusion}

We have studied the decay of Rydberg excitations in a cold $\mathrm{Rb}$ gas and find no evidence for the dramatic decrease in lifetimes predicted by Wang et al. [44]. The decay rates and population redistribution we observe are consistent with a model that considers only spontaneous emission from, and blackbody redistribution within, isolated atoms. In our experiments, a small electric field in the interaction region ejects any free electrons or ions from the excitation volume, preventing ionization or population transfer due to interactions with charged particles. In addition, the lack of spatial overlap between the trapping lasers and the Rydberg excitation laser well outside of the cold atom cloud ensures that there is no Rydberg excitation within an extended volume of lower-density, background $\mathrm{Rb}$ atoms in the chamber. Suppression of superradiant emission is likely due to variations in transition energies across the cold Rydberg atom sample. For initial $s$ states, these variations are dominated by inhomogeneities in DD exchange interactions within the random ensemble. Such inhomogeneities will necessarily be present in any measurement involving a large number of atoms where the separation between atoms is not well defined. For initial $p$ states, the suppression is likely due to a combination of DD exchange and electric field inhomogeneities. 


\section{Chapter 4}

\section{Dipole-Dipole Resonance Line Shapes in a Cold Rydberg Gas}

This chapter details measurements of dipole-dipole resonance line shapes in cold $\mathrm{Rb}$

Rydberg atoms in a MOT. This work was originally published in Physical Review A and can be found in Ref. [130]. The work from Ref. [130] has been updated to include more recent measurements examining the relationship between interaction time and resonance width, leading to greater insight on the contributions of many-body interactions to our two-body model.

\subsection{Introduction}

Because of their large transition dipole moments, Rydberg atoms are greatly affected by weak electric fields, including the multipole fields of neighboring atoms [8]. Accordingly, interactions between Rydberg atoms can be quite strong, coupling electronic and center-of- 
mass degrees of freedom at large internuclear separations. Rydberg-Rydberg interactions were originally studied in the context of collisions in thermal samples [8]. More recently, however, attention has turned to the exploration and control of the coherent couplings that exist between Rydberg atoms in (nearly) frozen gases, where the thermal kinetic energy of the atoms is less than their mutual interaction energies [9-39]. Such interactions enable a variety of few- and many-body quantum phenomena as well as potential applications in quantum information [12,40-43]. Typically, dipole-dipole (DD) effects dominate the atom-atom interaction when the spacing between the Rydberg atoms is much larger than the radial extent of the electronic wave function on individual atoms.

The degree to which the DD coupling between Rydberg atoms influences their behavior depends sensitively on the energy level structure of the individual atoms. Given their large polarizabilities, it is straightforward to manipulate the interactions between Rydberg atoms by applying static or pulsed electric fields. Stark-tuned, (Förster) resonant energy transfer (RET) reactions have been studied in both thermal $[8,134,136]$ and cold Rydberg gases $[10,11,13,15,16,19-21,23,24,29,36,38]$, and are perhaps the simplest example of electric-field controlled DD interactions involving Rydberg atoms. As an example of a RET process, consider two identical atoms $A$ and $B$, separated by a distance $R$ and initially in the same Rydberg state $|P\rangle$. Direct electronic energy transfer from $A$ to $B$ can efficiently occur, with little or no center-of-mass translational energy exchange, if there exist two states, $|S\rangle$ and $\left|S^{\prime}\right\rangle$, with energies $E_{S, P} \simeq-E_{S^{\prime}, P}$ relative to $|P\rangle$. Assuming $|S\rangle,|P\rangle$, and $\left|S^{\prime}\right\rangle$ are adjacent Rydberg levels with approximately the same principal quantum number $n$, the transition matrix elements $\mu_{A}=\left\langle S\left|r_{A}\right| P\right\rangle$ and $\mu_{B}=\left\langle S^{\prime}\left|r_{B}\right| P\right\rangle$ are large (scaling as $n^{2}$ ) and energy transfer from $A$ to $B$ is facilitated by a DD-interaction $V_{D D} \sim \mu_{A} \mu_{B} / R^{3}$ (atomic 
units are used unless otherwise noted) [8]. Here, $r_{A}$ and $r_{B}$ are the distance between the electron and nucleus on atoms $A$ and $B$, respectively. The energy transfer results in the excitation of atom $B$ from $|P\rangle$ to $\left|S^{\prime}\right\rangle$ and simultaneous deexcitation of atom $A$ from $|P\rangle$ to $|S\rangle$. The process is resonant, and most efficient, when the applied field is tuned to a value $F=F_{0}$ where the detuning $\delta=E_{S, P}+E_{S^{\prime}, P}=0$.

In the context of collisions it makes sense to discuss the DD interaction between a pair of atoms in terms of RET between the individual atoms. However, in a frozen gas, the DD interaction between two atoms is more conveniently discussed as a coherent coupling between molecular, or atom-pair states. For the example in the preceding paragraph, the relevant (uncoupled) pair states at large $R$ are $|P\rangle|P\rangle$ and $|S\rangle\left|S^{\prime}\right\rangle$. At smaller $R$, the coupled atoms are described by eigenstates which are linear combinations of the two uncoupled pair states. Through the DD interaction, the probability amplitude initially in $|P\rangle|P\rangle$ can be coherently transferred to $|S\rangle\left|S^{\prime}\right\rangle$ (and back) at a rate, and with a maximum probability, that depends on $\mu_{A}, \mu_{B}, R$, and $\delta$. For samples involving more than two atoms, the coupling between non-nearest-neighbor atoms complicates the eigenstate composition and the coherent population transfer processes. Indeed, measurements of Rydberg population transfer probability, as a function of the detuning $\delta$ from DD resonance, have provided evidence that many-body interactions play an important role in the coupled-atom dynamics in large ensembles $[10,11]$. Also, clear changes in resonance "line shapes" have been observed as the number of interacting atoms increases from 2 to several [29].

Using line shape measurements for the $32 p_{3 / 2} 32 p_{3 / 2} \leftrightarrow 32 s 33 s$ DD resonance in $\mathrm{Rb}$, we clarify the respective roles of nearest- and beyond nearest-neighbor interactions in resonantly coupled systems, and show that the line shapes contain information on the 
position correlation function of Rydberg atoms in a cold random ensemble.

\subsection{Experimental Procedure}

In the experiments, ${ }^{85} \mathrm{Rb}$ atoms at $\sim 70 \mu \mathrm{K}$ in a magneto-optical trap (MOT) (described in Section 2.2) are photoexcited from the $5 p_{3 / 2}$ Rydberg state in the presence of a weak electric field, $F \simeq 15 \mathrm{~V} / \mathrm{cm}$. The field mixes a small amount of $n s$ and $n d$ character into the $p$ state to enable the excitation, but detunes the atoms sufficiently far from DD resonance so that, initially, there is negligible interaction between them. The atoms are then exposed to a fast-rising "tuning" electric field pulse. The tuning pulse alters the energy differences $\delta$ between the $32 p_{3 / 2} 32 p_{3 / 2}$ and $32 s 33 s$ pair states, and projects the initial $32 p$ population onto the coupled pair states. The system is allowed to evolve throughout the duration $\tau$ of the tuning pulse. State-selective field ionization (SSFI) is then employed to measure the population transferred to $32 \mathrm{~s} 33 \mathrm{~s}$ pairs as a function of the tuning field strength and Rydberg density [8].

A $482 \mathrm{~nm}$ laser pulse directly excites atoms from the $5 p_{3 / 2}$ trap state to the $32 p_{3 / 2}$ Rydberg state. The experiments are performed using either a "long," narrow-band ( $\sim 1$ $\mathrm{MHz})$ or "short," broader-band ( $\sim 100 \mathrm{MHz})$ Rydberg excitation pulse. The long pulse is created using the method described in Section 2.1.1 in which an AOM chops a $3 \mu \mathrm{s}$ excitation pulse, with $\sim 1 \mu$ s rise and fall times, from a narrow band $(\sim 1 \mathrm{MHz})$ continuous wave diode laser. Alternatively, a Pockels cell is employed to slice a short $10 \mathrm{~ns}$ pulse from the diode laser, and that pulse is amplifed in dye using the $10 \mathrm{~ns}, 355 \mathrm{~nm}$ third harmonic of a pulsed Nd:yttrium-aluminum-garnet (YAG) laser (see Section 2.1 for a description of 
the Pockels cell, dye amplifier, and the Nd:YAG laser). In both cases, the $482 \mathrm{~nm}$ beam is focused into the $0.4 \mathrm{~mm}$ diameter MOT using a $350 \mathrm{~mm}$ focal length lens, exciting a cylindrical atomic volume with a diameter of $\sim 0.1 \mathrm{~mm}$ and a length of $0.4 \mathrm{~mm}$.

The laser excitation, energy tuning, and field ionization of the Rydberg atoms are facilitated by the application of pulsed and static voltages to two pairs of thin, parallel, stainless steel rods that are arranged around the cold atom cloud in a rectangular array. The field produced by the rods at the position of the MOT is proportional to the voltage difference between rod pairs and is quite uniform, with a variation of $0.47 \%$ over the atom cloud. The voltage pulse which produces the tuning field has fast $(2 \mathrm{~ns})$ rise and fall times and is produced by using an arbitrary wave-from generator (AWG) followed by a DC-coupled, pulse amplifier (described in Section 2.3.3). When the rise and fall times of the tuning pulse are set to the minimum of $2 \mathrm{~ns}$, small oscillations are observed during the nominally constant portions of the pulse waveform. These parasitic oscillations give rise to sidebands in the applied electric field that can lead to additional population transfer away from the peak of the resonance line shape (shown in Figure 4.1). To remove these oscillations, an exponential rising and falling pattern, with a rise and fall time of $\sim 10 \mathrm{ns,} \mathrm{is}$ used.

At the end of the interaction period defined by the tuning pulse, a high-voltage ramp applied to the field rods ionizes Rydberg atoms in the interaction region, propelling them toward a microchannel plate (MCP) detector (see Section 2.3.2). Different Rydberg states ionize at different times during the ramp [8]. Therefore, in principle, populations in different states can be distinguished in the time-dependent signal from the MCP detector. In practice, to obtain better temporal separation between the signal corresponding to the populations in 
the initial $\left(32 p_{3 / 2} 32 p_{3 / 2}\right)$ and final $(32 s 33 s)$ pair states, the maximum ionization field is set just above the threshold for ionizing $32 p_{3 / 2}$. As a result, we do not ionize atoms in $32 s$, and detect only half of the atoms (those in $33 s$ ) in each $32 s 33 s$ pair.

By recording the population transfer to $33 \mathrm{~s}$ as a function of the tuning field, we obtain the resonance line shape (see Figure 4.1). Line shapes are measured over a range of Rydberg densities, $2 \times 10^{8}<\rho<3 \times 10^{9} \mathrm{~cm}^{-3}$. The density is varied by adjusting the current applied to the getters that supply Rb to the MOT. The density of atoms in the MOT is determined, to within $30 \%$, by combining measurements of the spatial dimensions of the atom cloud size via direct imaging with a CCD camera with measurements of the radiated fluorescence using an optical power meter. The relative uncertainty between density measurement is less than $30 \%$. By saturating the Rydberg excitation using a sufficiently high laser fluence, we ensure that approximately one-third of the atoms in the excitation volume are excited to Rydberg states (see Section 2.2.3). Full line shapes were measured over a range of densities for $\tau=200 \mathrm{~ns}$ and $1 \mu \mathrm{s}$, for the exponential rise and fall patterns, and $\tau=15 \mu$ s for the step function rise and fall patterns.

In addition to the field produced by the rods, an additional parallel "offset" field of $2.758 \pm 0.005 \mathrm{~V} / \mathrm{cm}$ contributes to the net electric field in the interaction region. The offset field is the result of imperfect shielding of the MCP detector and is less homogeneous than the rod field. As discussed in more detail below, the measured line shapes can be used to characterize the variation in this offset field over the atomic ensemble. 
CHAPTER 4. DIPOLE-DIPOLE RESONANCE LINE SHAPES INA COLD RYDBERG GAS76

\subsection{Experimental Results}

Figure 4.1 shows the population transferred to $33 \mathrm{~s}$ as a function of the strength of the applied tuning field at three different densities. These line shape data exhibit several notable features. First, the functional form of the resonance lines change from something resembling a Gaussian at low density to a cusp, characterized by a narrow central peak with broad wings, at higher density. As described in detail in Section 4.4, the cusp line shape reflects the random variation in the interatomic spacing $R$ within the random ensemble.

Second, the maxima of the three line shapes appear at (slightly) different applied tuning fields, at values closer to $9.8 \mathrm{~V} / \mathrm{cm}$ than to the expected value, $F_{0}=12.5 \mathrm{~V} / \mathrm{cm}$, at which the resonance condition, $\delta=0$, is fulfilled for atoms initially in the $32 p_{3 / 2}\left|m_{j}\right|=3 / 2$ state. Here $m_{j}$ is the projection of total electronic angular momentum on the z-axis. As noted previously, the nominal $2.758 \mathrm{~V} / \mathrm{cm} \mathrm{MCP}$ offset field adds to the applied field from the rods, shifting the apparent resonance field. The variation in the peak position for different data sets is not caused by the different densities at which the data were taken, but is due rather to the inhomogeneity in the offset field and slight differences in the position of the Rydberg excitation laser within the MOT for different data runs. This was confirmed by measuring the line shape position as a function of excitation laser displacement within the MOT. The spatial variation in the offset field within the Rydberg excitation beam is responsible for the Gaussian, inhomogeneously broadened line shapes observed at low density.

Third, the widths of the line shapes grow linearly with increasing density. Figure 4.2 shows the full width at half maximum (FWHM), $\Delta$, of the measured resonances profiles as a function of Rydberg density. To convert the resonance widths (which are measured in units of field) to units of energy, we first measure the Stark shifts of the $32 p_{3 / 2}, 32 s$, 
and $33 s$ states as a function of applied field in the vicinity of the resonance. We then use those spectroscopic data to compute the detuning $\delta$ as a function of the applied field (see Fig. 4.3). We find that near $F_{0}, \delta$ varies approximately linearly with $F$ with a slope of $170 \mathrm{MHz} /(\mathrm{V} / \mathrm{cm})$. Note that since the tuning field, not the excitation laser, determines the detuning of the atoms from resonance, the laser bandwidth does not factor into the measured resonance widths.

\subsection{Analysis and Discussion}

The resonance line shapes carry information on the relative strength of nearest- and beyond nearest-neighbor interactions, the distribution of atom separations, and field inhomogeneities in the Rydberg ensemble. In order to extract that information, we must identify how each of these influences the different features in the observed profiles. To that end, we first consider the form of the line shape associated with a pair of stationary atoms, with a well-defined separation, coupled via a (near) resonant $32 p 32 p \leftrightarrow 32 s 33 s$ DD interaction. We diagonalize the Hamiltonian in the presence of the DD interaction, restricting the pair-state basis to those levels which are degenerate at resonance. This basis includes numerous states with different azimuthal quantum numbers $m$ for the individual atoms and different values of total electronic angular momentum and its projection on the internuclear axis $[5,134]$. However, ignoring spin, the problem reduces to an equivalent two-level system, involving two pair basis states, $|1\rangle$ and $|2\rangle$, with a DD coupling of $V_{d d}=\frac{2 \mu_{A} \mu_{B}}{\sqrt{3} R^{3}}$ between them [5]. Here $|1\rangle$ is a linear combination of $32 p 32 p$ states, $|2\rangle$ is an equal admixture of $32 s 33 s$ and $33 s 32 s$, and $\mu_{A}$ and $\mu_{B}$ are defined as in Section 4.1. 


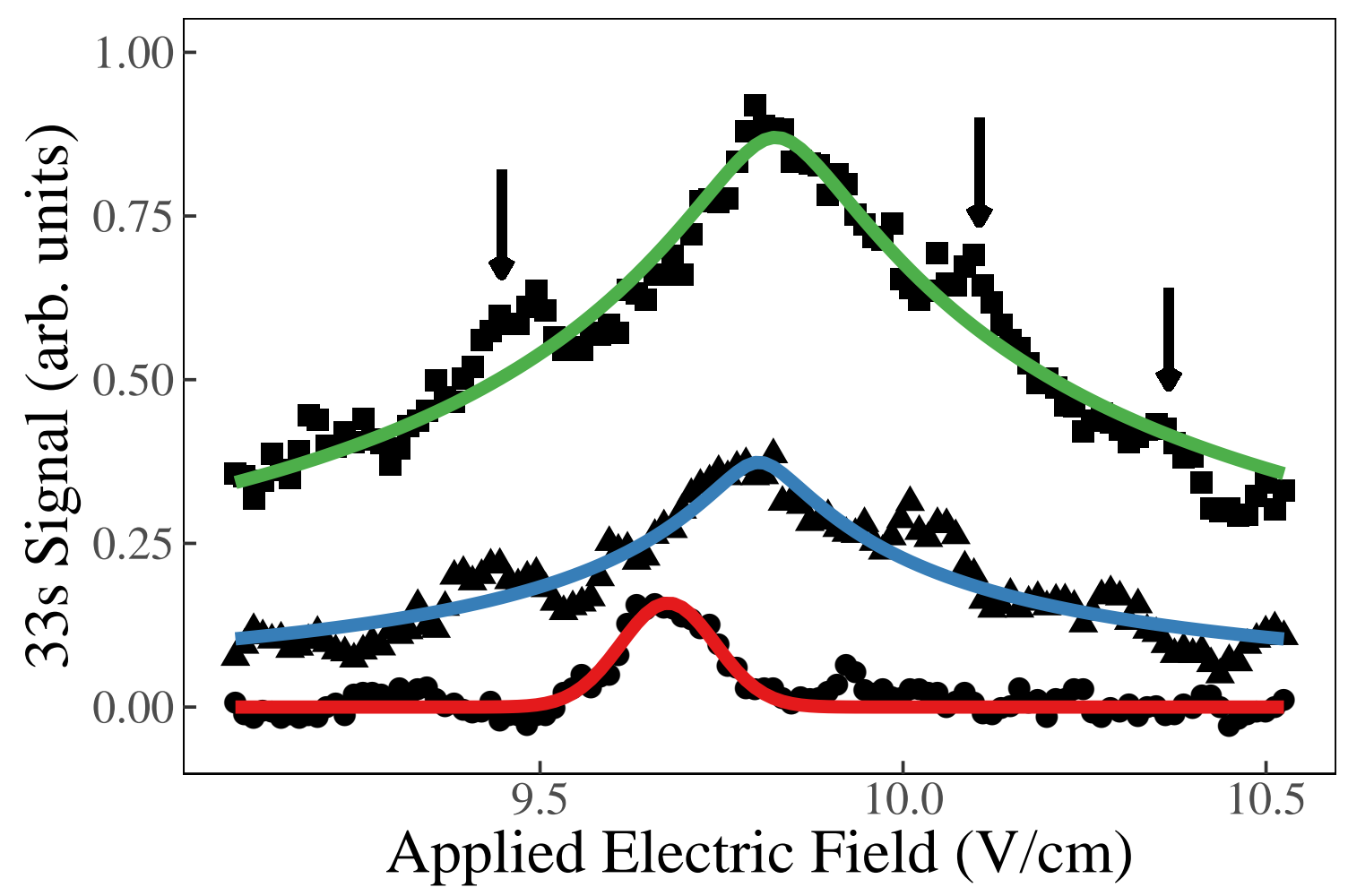

Figure 4.1: $32 p_{3 / 2} 32 p_{3 / 2} \rightarrow 32 s 33 s$ DD-resonance line shapes, showing population transfer to $33 \mathrm{~s}$ as a function of applied tuning field at various Rydberg densities: $\rho=2 \times 10^{8}$ (filled circles); $\rho=1 \times 10^{9}$ (filled triangles); and $\rho=3 \times 10^{9} \mathrm{~cm}^{-3}$ (filled squares). These line shapes were measured using a step function rising and falling edge for the tuning pulse and a tuning pulse length of $\tau=15 \mu \mathrm{s}$. The measured signals are not individually normalized, so the relative heights of the profiles reflects the difference in resonant transition probability. The baseline, corresponding to zero population transfer, is the same for the three data sets. The horizontal axis shows the applied tuning field due to the rods. The resonance line centers are shifted from the expected resonance condition, $F_{0}=12.5 \mathrm{~V} / \mathrm{cm}$, due to the presence of the MCP offset field described in Sections 3.4 and 4.3. The three data sets are acquired with the excitation beam focused at (slightly) different locations within the MOT. The relative shifts of the line centers are due to the variation in the offset field within the FWHM of the atom cloud. As described in Section 4.4, the inhomogeneity in the offset field is also responsible for the Gaussian line shapes observed at low Rydberg density. The solid (red) line through the lowest density data is the best Gaussian fit of the inhomogeneously broadened line shape. The solid (blue and green) lines through the higher density data are fits to the cusp line shape expected for a random ensemble, as described in Section 4.4. The small peaks indicated by arrows on either side of the resonance data are sidebands due to oscillations in the tuning pulse and vanish when an exponential rising and falling pattern is applied to the tuning pulse. 


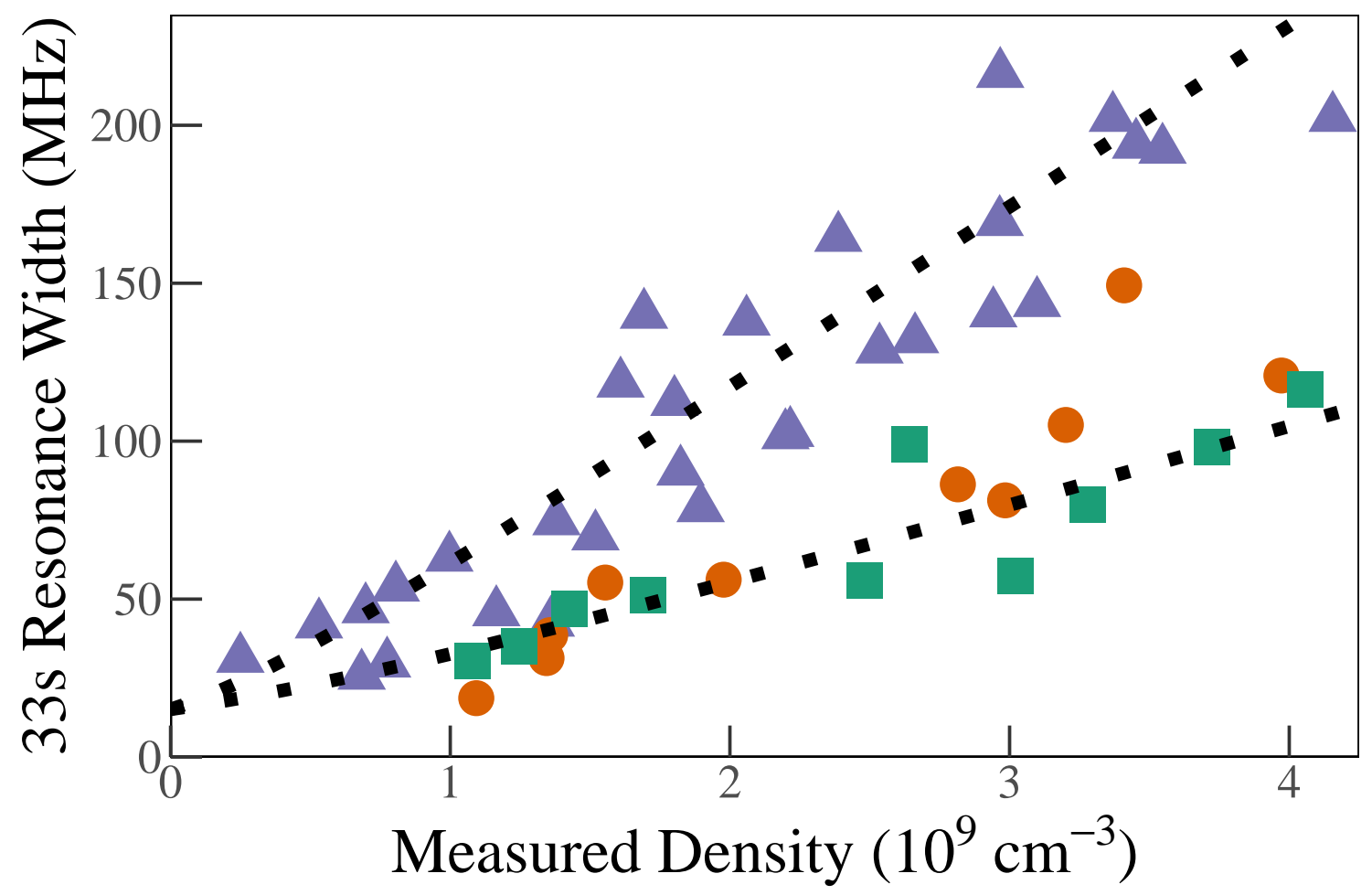

Figure 4.2: Resonance width as a function of Rydberg density. Measured widths are shown as points with the shapes showing different tuning pulse lengths $\tau$ : $200 \mathrm{~ns}$ (green squares), $1 \mu \mathrm{s}$ (orange cirlces), $15 \mu \mathrm{s}$ (purple triangles). The two dotted lines are the calculated width as described in Section 4.4 (lower) and the calculated width multiplied by a factor of 2 (upper) assuming only nearest-neighbor interactions and $15 \mathrm{MHz}$ of inhomogeneous broadening due the offset field. The points for $\tau=15 \mu$ s include data taken with both a long $3 \mu$ s excitation laser pulse and a short $10 \mathrm{~ns}$ excitation laser pulse. No significant difference in the profile widths for the long and short pulse excitations is expected or observed. 


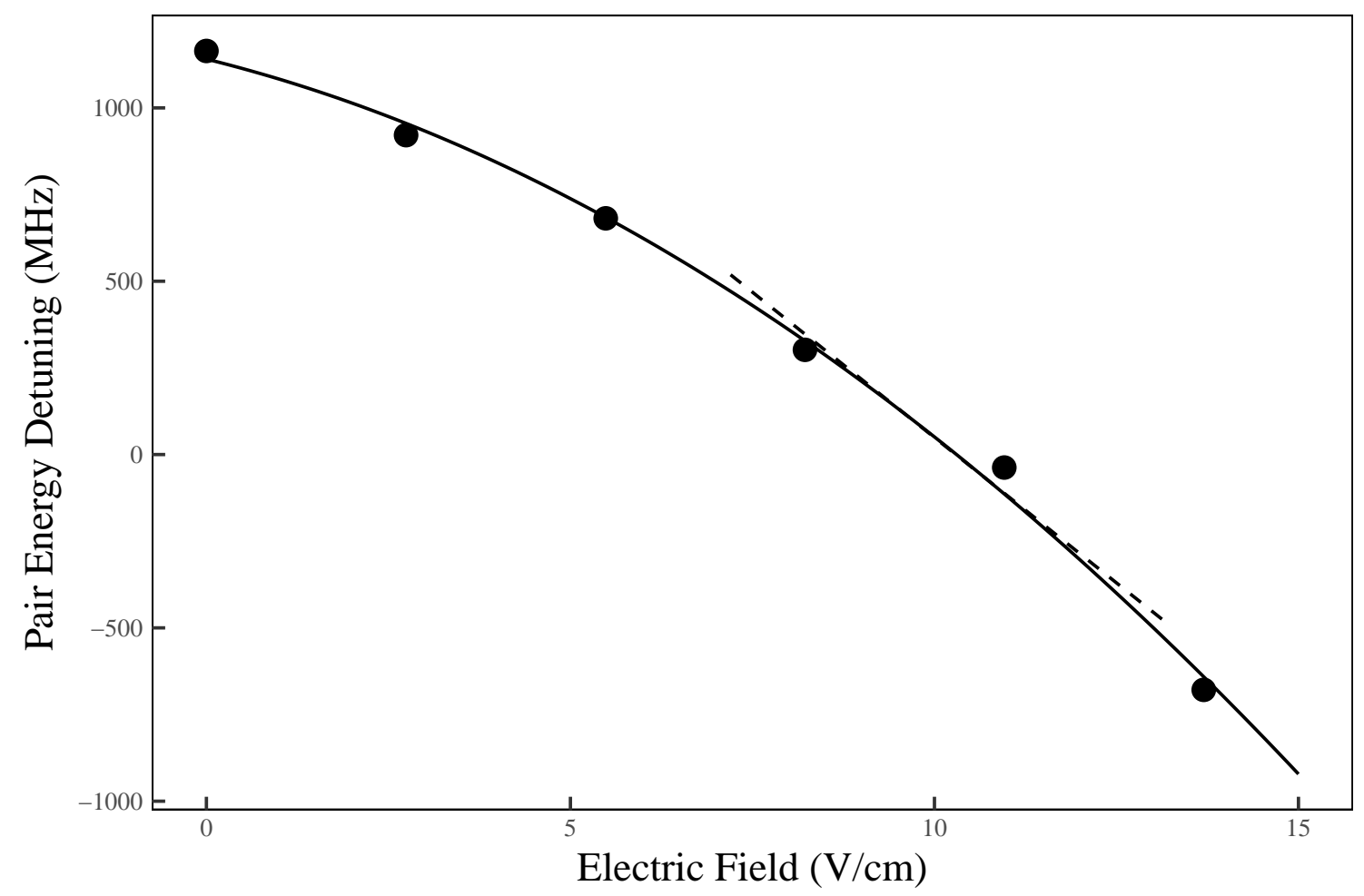

Figure 4.3: Pair-energy detuning from resonance as a function of applied electric field. The filled circles are experimentally determined values of $\delta$ obtained from Stark shift measurements for the $32 p_{3 / 2}\left|m_{j}\right|=3 / 2,32 s$, and $33 s$ levels as a function of the applied field. At these low fields, the energies of all three levels shift quadratically with the field. The solid line is a quadratic fit to the data. Near resonance, the variation in $\delta$ is approximately linear with a slope of $170 \mathrm{MHz} /(\mathrm{V} / \mathrm{cm})$ (dashed line). 
Diagonalizing the effective two-level Hamiltonian, one obtains the eigenstates

$$
\begin{aligned}
& |+\rangle=\cos \theta|1\rangle+\sin \theta|2\rangle, \\
& |-\rangle=-\sin \theta|1\rangle+\cos \theta|2\rangle,
\end{aligned}
$$

where $\tan 2 \theta=2 V_{D D} / \delta$. For these states $\theta=0$ for fields $F \ll F_{r e s}, \theta=\pi / 4$ for $F=F_{\text {res }}$, and $\theta=\pi / 2$ for $F \gg F_{\text {res }}$. These eigenstates have energies $E_{ \pm}=(\delta \pm \Gamma) / 2$, where $\Gamma=\sqrt{\delta^{2}+4 V_{D D}^{2}}$, and exhibit a standard avoided level crossing as a function of $\delta$, with an energy separation $\Delta E=E_{+}-E_{-}=2 V_{D D}$ at $\delta=0$. At large detunings from resonance, $|+\rangle$ and $|-\rangle$ have only $p$ - and $s$-character, respectively. Thus, the initial laser excitation, which is performed in an electric field for which atom pairs are far-detuned from the $32 p 32 p \leftrightarrow 32 s 33 s$ resonance, creates only $32 p$ atoms, thereby populating only $|+\rangle$. At $t=0$, the fast rising tuning-field pulse then projects the $32 p 32 p$ atom pairs into a coherent superposition of $|+\rangle$ and $|-\rangle$.

Initially, the time-dependent electronic wave function of each atom pair has only $32 p 32 p$ character. However, assuming coherence is maintained, the pair state evolves as a wave packet in the uncoupled basis, according to the two-level Rabi formula:

$$
\Psi(t)=[\cos (\Gamma t / 2)-i \eta \sin (\Gamma t / 2)]|1\rangle+i \chi \sin (\Gamma t / 2)|2\rangle
$$

where $\Gamma$ is the effective Rabi frequency, $\chi=2 V_{D D} / \Gamma$, and $\eta=\delta / \Gamma$ is a scaled detuning.

The principal signature of the evolution of this wave packet is the coherent transfer of population from $|1\rangle$ to $|2\rangle$, i.e., from $p$ - to $s$-character. According to Eq. 4.2, the probability of finding an atom in the $33 s$ state at a time $t$ following the start of the tuning pulse is, 
$\mathcal{P}_{0}=\chi^{2} \sin ^{2}(\Gamma t / 2)$. The temporal modulations in $\mathcal{P}_{0}$, predicted for a single atom pair, can be viewed as Rabi oscillations due to the coupling between the pair states $|1\rangle$ and $|2\rangle$ or, alternatively, as a quantum beat induced by the coherent excitation of the DD-dressed states $|+\rangle$ and $|-\rangle$. The amplitude of the Rabi oscillations, $\chi^{2}$, is a Lorentzian function of the detuning $\delta$ centered at $\delta=0$ with a FWHM, $\Delta=4 V_{D D}$.

Experimentally, we measure the number of $33 \mathrm{~s}$ atoms, produced via DD resonance, from a random ensemble of $32 p$ atoms. Within the ensemble, there is a broad distribution of atom separations and, therefore, a wide range of values for $V_{D D}$ and $\Gamma$ for different atom pairs. As a result, a monotonic increase and eventual saturation, but no Rabi oscillations, are actually observed in the $33 \mathrm{~s}$ population measured as a function of the interaction time, $\tau$. Accordingly, for sufficiently long $\tau$, one might expect the ensemble to exhibit a Lorentzian population transfer line shape (see Fig. 4.4) that is approximately equal to the time-averaged value of $\mathcal{P}_{0}$ for a single atom pair

$$
\mathcal{P}=\frac{1}{2} \chi^{2}=\frac{2 V_{D D}^{2}}{\delta^{2}+4 V_{D D}^{2}},
$$

with the values of $\chi$ and $V_{D D}$ computed using $R \simeq(2 \pi \rho)^{-1 / 3}$, the most probable nearestneighbor separation in the ensemble. Assuming only nearest-neighbor interactions, this approximation correctly predicts the FWHM of the resonance, $\Delta$, but it is a poor representation of the line shape overall.

To properly account for the variation in atom separations throughout the excitation volume, we integrate Eq. 4.3 over all $R$, weighting the contribution from each $R$ by the 


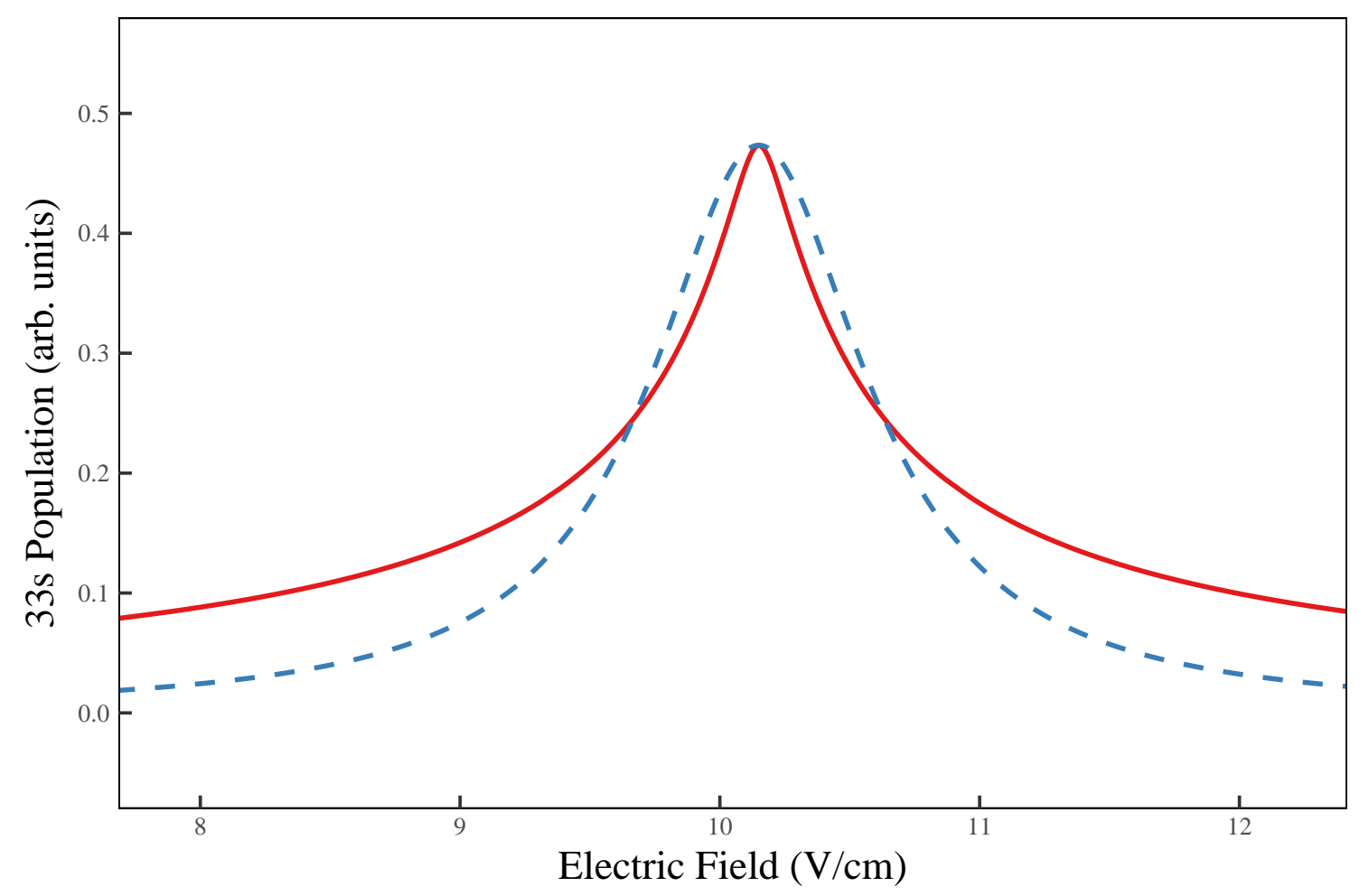

Figure 4.4: Comparsion of the cusp (solid line) and Lorentzian (dashed line) line shapes expected for ensembles with random [see Eq. 4.5] and uniform [see Eq. 4.3] atom separation, respectively. The Lorentzian profile assumes the most probable value of $R$ at the Rydberg density used to compute the cusp. Note the cusp's broad, large amplitude wings and relatively narrow central peak. 
nearest-neighbor distribution for a random ensemble [137]

$$
P(R)=4 \pi \rho R^{2} e^{-\frac{4}{3} \pi \rho R^{3}}
$$

The line shape resulting from the integration can be expressed in terms of standard functions as

$$
\mathcal{P}=\frac{1}{2} a\left\{\operatorname{Ci}(a) \sin a+\left[\frac{\pi}{2}-\operatorname{Si}(a)\right] \cos a\right\}
$$

where $a=16 \pi \rho \mu_{A} \mu_{B} /(3 \sqrt{3} \delta), \operatorname{Si}(x)=\int_{0}^{x} \frac{\sin u}{u} d u$ is the sine integral, $\operatorname{Ci}(x)=\gamma+\ln x+$ $\int_{0}^{x} \frac{\cos u-1}{u} d u$ is the cosine integral, and $\gamma \simeq 0.577216$ is Euler's constant [138]. As shown in Fig. 4.4, the line shape takes the form of a cusp which has a narrower central peak and significantly broader wings as compared to the Lorentzian profile computed at the same Rydberg density, but using a uniform atom separation equal to the most probable value of $R$. It is worth nothing, however, that the FWHM of the two line shapes

$$
\Delta \simeq 16 \pi \mu_{A} \mu_{B} / \sqrt{3}
$$

are identical, and as expected, are directly proportional to the Rydberg density. For the $32 p 32 p \leftrightarrow 32 s 33 s$ resonance, we compute $\mu_{A}=\langle 32 s|r| 32 p\rangle=964$ and $\mu_{B}=$ $\langle 33 s|r| 32 p\rangle=941$, giving $\Delta=26 \mathrm{MHz}$ for $\rho=1 \times 10^{9} \mathrm{~cm}^{-3}$.

In Fig. 4.1, the solid curves drawn through the data collected at $\rho=1 \times 10^{9}$ and $\rho=3 \times 10^{9} \mathrm{~cm}^{-3}$ are fits of Eq. 4.5 to those line shapes. The fits capture the primary features of the observed profiles when the weaker satellite resonances (sidebands due to the oscillations in the tuning pulse) in the data are ignored. At these densities, the "natural" width 
due to the DD interaction is the dominant contributor to the line shape, and inhomogeneous broadening due to the spatial variation in the offset field has a negligible effect. The broad wings of the line shapes distinguish them from the Lorentizans expected for an ensemble with well-defined atom separation. Conversely, for $\rho=2 \times 10^{8} \mathrm{~cm}^{-3}$, the natural width is less than the inhomogeneous width. As a result, in convolution, the measured profile is well represented by a Gaussian with negligible wings far from resonance.

The dashed curves in Fig. 4.2 are the FWHM of simulated profiles constructed by convoluting the cusp of Eq. 4.5, whose natural width increases proportionally to the Rydberg density, with a fixed-width (15 MHz) Gaussian. The Gaussian is included to model the effects of inhomogeneous broadening associated with the spatial variation in the applied electric field, and its FWHM is chosen to be in accord with the resonance widths measured at the lowest densities. In the following, we argue that the principal source of the observed inhomogeneous broadening is the gradient in the offset field produced by the MCP (as noted in Chapter 3).

As noted previously, the variation in the rod field over the interaction region is only $0.47 \%$, leading to a variation in $\delta$ of approximately $1 \mathrm{MHz}$ for tuning fields near the resonance condition. While there is a magnetic field gradient in the interaction region due to the MOT coils, the variation in $\delta$ due to Zeeman shifts is also $\sim 1 \mathrm{MHz}$. Both of these inhomogeneities are essentially negligible when taken in quadrature with the other sources of broadening that lead to the combined $15 \mathrm{MHz}$ inhomogeneous width observed at very low density. Given the detuning slope of $170 \mathrm{MHz} /(\mathrm{V} / \mathrm{cm})$ near resonance, an offset field variation of $0.085 \mathrm{~V} / \mathrm{cm}$ accounts for the $15 \mathrm{MHz}$ minimum width. Interestingly, a very similar offset field variation, $0.081 \mathrm{~V} / \mathrm{cm}$, when taken in quadrature with magnetic field inhomogeneity, also explains the 
4.2 $\mathrm{MHz}$ minimum width observed in independent measurements of the $25 s 33 s \leftrightarrow 24 p 34 p$ resonance, using the same apparatus and experimental geometry [36]. The latter resonance has considerably different tuning properties, including a detuning slope of $51 \mathrm{MHz} /(\mathrm{V} / \mathrm{cm})$ and resonant field of $3.4 \mathrm{~V} / \mathrm{cm}$, making it highly unlikely that the agreement between the two field variation determinations is coincidental.

Fig. 4.2 shows two simulated line widths. The lower dashed line represents the natural line widths predicted by Eq. 4.6 for the cusps used in the simulations. The upper dashed line is the calculated width assuming double the natural line widths predicted by Eq. 4.6. Fig. 4.2 show that we have a good agreement between our calculation and measurements for $\tau \leq 1 \mu \mathrm{s}$. For longer pulses, we observe a broadening of a factor of 2 beyond the two-body stationary model. One possible source of discrepancies between the measurements and the simulated widths could be uncertainty in the measured Rydberg density. However, the measurements made at short times agree well with the model and the density only has a $30 \%$ uncertainty with a relative uncertainty of less than $30 \%$. This makes it unlikely to be a major contributor to the broadening observed at longer times. We must consider two other possible broadening sources: beyond nearest-neighbor interactions and atom motion.

Historically, beyond nearest-neighbor interactions were considered the primary contributor to broadening in dipole-dipole resonance line shapes. The first studies of RET in a cold Rydberg gas $[10,11]$ reported measured widths that were much (up to two orders of magnitude) broader than expected for isolated pairs of atoms. It was suggested that rapid diffusion of the population, away from an interacting nearest-neighbor pair to other nearby atoms, rapidly occurred via exchange or "hopping" interactions of the form $|P\rangle|S\rangle \rightarrow|S\rangle|P\rangle$. Subsequent experiments verified the excitation diffusion process $[13,15]$ in the absence of 
other strong interactions. However, more detailed simulations $[9,24]$ of tunable, resonant population transfer in a many-atom system showed that the primary DD coupling between nearest neighbors could suppress the diffusion process. As a result, the inclusion of exchange interactions resulted in only a modest $(\sim 50 \%$ [9]) increase in the resonance width. According to [9], the characteristic time for diffusion is $t_{c}=(1 / 10)(1 / \bar{V})$ where $\bar{V}=\mu^{2} n$, $\mu$ is the transition matrix element for the diffusion interaction, and $n$ is the average density of the gas. This gives $t_{c} \approx 10 \mathrm{~ns}$ for both the $32 p 32 s \leftrightarrow 32 s 32 p$ and $32 p 33 s \leftrightarrow 33 s 32 p$ diffusion interactions. With interactions times $\tau \gg t_{c}$, we do not expect broadening due to diffusion to grow with interaction time, though the total population measured in the $|S\rangle$ states may grow. Since broadening from the diffusion process does not grow with increased interaction time, it does not explain the tuning pulse length dependent broadening and is not likely a contributor to the measured broadening.

Our final source of broadening to examine is relative atom motion, which is neglected in our model. For $\rho=2 \times 10^{9} \mathrm{~cm}^{-3}$, the most probable atom separation is $R \sim 4 \mu \mathrm{m}$ and the rms relative velocity between two $70 \mu \mathrm{K}$ atoms is $v_{r m s}=0.2 \mu \mathrm{m} / \mu \mathrm{s}$. Depending on the direction of relative motion, in a $\tau=15 \mu$ s interval, the separation between typical nearest neighbors changes by $15 \%$ to $75 \%$. In comparison to shorter interaction times, $\tau=$ $200 \mathrm{~ns}(1 \mu \mathrm{s})$, the separation between typical nearest neighbors changes by $0.2 \%(1 \%)$ to $1 \%(5 \%)$. For a simple, qualitative example of how atom motion might broaden the line shapes, let us assume that we have a cloud of atoms in a random distribution as described in Eq. 4.4. Close to the dipole-dipole resonance, all or most of the atoms are at separations that allow for participation in the RET interaction. In the wings of the resonance, only close together atoms will undergo the RET interaction. Thus, at longer time scales, there 
is a greater chance for atoms that were originally far apart and unable to interact to move closer together and undergo the RET interaction, leading to broader line shapes for longer interaction times. At short interaction times, the atoms are essentially frozen and only interact with their nearest neighbor at the beginning of the interaction time. As shown in Fig. 4.2, the $\tau=15 \mu$ s data falls approximately a factor of 2 above the simulated widths from Eq. 4.6. Clearly, there is a time-dependent broadening effect that is most likely attributable to atomic motion.

The broadening attributed to atomic motion could be further clarified by varying the temperature of the MOT and measuring the resonance line shape widths at a fixed interaction time. If the temperature of the MOT can be increased, the increased motion at shorter interaction times should lead to a broadening in the widths. If the temperature of the MOT can be decreased, by evaporative cooling processes for example, the decreased motion at longer interaction times should lead to a reduction in the widths. Measurements made by Mary Kutteruf show that the MOT temperature is quite independent of the atom density and trap/re-pump laser power [5], making it difficult to adjust the temperature of the MOT and maintain the same density for comparison.

\subsection{Conclusion}

We have measured, as a function of atom density, the line shapes associated with $32 p_{3 / 2} 32 p_{3 / 2} \rightarrow 32 s 33 s$ resonant population transfer in a cold Rb Rydberg gas. The line shapes are cusplike at high density, reflecting the random nearest-neighbor separation in the MOT, and are well reproduced by a closed form expression based on a two-body interaction 
model. The resonance widths agree with the model with shorter tuning pulse lengths, $\tau=$ $200 \mathrm{~ns}$ and $1 \mu \mathrm{s}$, overlapping well with the model and with longer tuning pulse lengths, $\tau=$ $15 \mu \mathrm{s}$, agreeing up to a factor of 2 , confirming that beyond nearest-neighbor processes such as excitation diffusion do not influence the population transfer rate to the degree previously indicated. Rather, atom motion appears to be the primary contributor to the line shape broadening at larger interaction times. At a low density the change in the line shape from a cusp to Gaussian form allows us to characterize the electric field inhomogeneitiy in the interaction region. In the future, similar resonance line shape analyses may make it possible to distinguish random from uniform atom distributions, perhaps providing a method to visualize changes in the position correlation function with the application of controlled DD forces between atoms (as proposed in [22] and explored in Chapter 5). 


\section{Chapter 5}

\section{Towards Controlling Minimum Atom Separation in a Cold Gas}

\subsection{Introduction}

Dipole-dipole (DD) interactions between Rydberg atoms can be strong at large distances due to the $n^{2}$ scaling of the dipole moment. In the previous chapters, we examined how DD interactions can shift the energies of the electronic states of atoms (Chapter 3) and we examined how dipole-dipole mediated resonant energy transfer (RET) can change the wavefunction character of pairs of Rydberg atoms as a function of an applied electric field (Chapter 4). As part of the discussion of RET line shapes in Chapter 4 we examined the effect that nearest-neighbor interactions, atomic motion, and atomic density have on the transfer of population between, and energy of, atoms in our MOT. We are interested in exploring control over the center of mass motion of atoms in a Rydberg-dressed dipole- 
dipole coupled system. Specifically, we examine systems where DD interactions might allow us to set a minimum separation between atoms in the MOT, perhaps resulting in longer range order.

Work on laser trapping and cooling over the past few decades has given us many new experimental techniques. Among these techniques is Rydberg dressing, where optical excitation mixes Rydberg character into the ground state of some atomic system [139]. In 2007, Wall et al. proposed a method for using Rydberg dressing to control the minimum separation between nearest-neighbor atoms in a cold ensemble [22]. Since that time, many other groups have explored the concept of Rydberg dressing for various applications. For example, Rydberg dressing has been used to study strongly correlated phases with reduced decoherence [140], quantum magnetism and topological ordering [109], excitation dynamics [141], the coherent migration of electronic excitation [142], and the creation of a single-photon transistor [38]. Many of these experiments exploit the strong long-range interactions between Rydberg atoms while also benefiting from enhanced life times due to only a small fraction of Rydberg character being mixed into the ground state.

In this chapter, we make a first attempt to implement the proposal [22] to use Rydberg dressing to manipulate atom separations in a MOT. Due to their strong long-range interactions, Rydberg atoms can exert mechanical forces on one another. As two Rydberg atoms come together or move apart, the $R$-dependent $\mathrm{DD}$ interaction results in a change in the electronic energy of the atoms. Any gain (loss) in electronic energy is accompanied by a subsequent loss (gain) in the center-of-mass motion of the atoms in the pair.

Figures 5.1 and 5.2 show a possible simple model system for controlling atom motion with DD interactions. More complete models are considered by Wall et al. and in Section 
5.4. Let us assume that we have cold ${ }^{85} \mathrm{Rb}$ atoms in a MOT that uses the $5 s_{1 / 2} \leftrightarrow 5 p_{3 / 2}$ trapping cycle described in Section 2.2. Atom pairs are illuminated by a "control" laser with detuning $\Delta$ to the blue (i.e. higher frequency) of the Stark-shifted single-atom $5 p_{3 / 2} \rightarrow$ $32 p_{3 / 2}$ Rydberg transition. Figure 5.1(a) shows the energies of the control-laser-dressed states, as a function of atom pair separation $R$, for 0 control-laser intensity and coupling. The zero-energy point is defined as the upper, solid, horizontal line corresponding to the $5 p_{3 / 2} 5 p_{3 / 2}$ trap state. The other solid horizontal line corresponds to the singly-excited Rydberg state (either $5 p_{3 / 2} 32 p_{3 / 2}$ or $32 p_{3 / 2} 5 p_{3 / 2}$ ). The two repelling curves correspond to doubly-excited Rydberg states with a $\sim R^{-3}$ dependent DD interaction. The dashed horizontal line gives the energy of the doubly-excited Rydberg state for atoms at infinite separation. The DD interaction we consider is the same as in Chapter 4, the resonant $32 p_{3 / 2} 32 p_{3 / 2} \leftrightarrow 32 s 33 s$ DD reaction. With the application of a small electric field, this reaction can be brought into resonance, with $2 E_{32 p_{3 / 2}}-\left(E_{32 s}+E_{33 s}\right)=0$. As in Chapter 4, the pair-state basis includes numerous states with azimuthal quantum numbers $m$ for the individual atoms and different values of total electronic angular momentum and its projection on the internuclear axis. However, if we ignore spin, the problem reduces down to an equivalent two-level system with a DD interaction potential of $V_{D D} \sim \frac{\mu_{A} \mu_{B}}{R^{3}}$ where $\mu_{A}=\left\langle 32 p_{3 / 2}|r| 32 s\right\rangle$ and $\mu_{B}=\left\langle 32 p_{3 / 2}|r| 33 s\right\rangle$ are dipole transition matrix elements and $R$ is the separation between pairs of atoms [5,134]. For 0 control laser intensity or coupling, the doubly-excited Rydberg state energy curves cross with that of the singly-excited Rydberg state and trap state energies. For the case of a laser coupling with a single-atom Rabi frequency $\Omega$, these crossings become avoided crossings (see Figure 5.1(b)).

Consider the upper dressed state which has no change in energy at large separations 


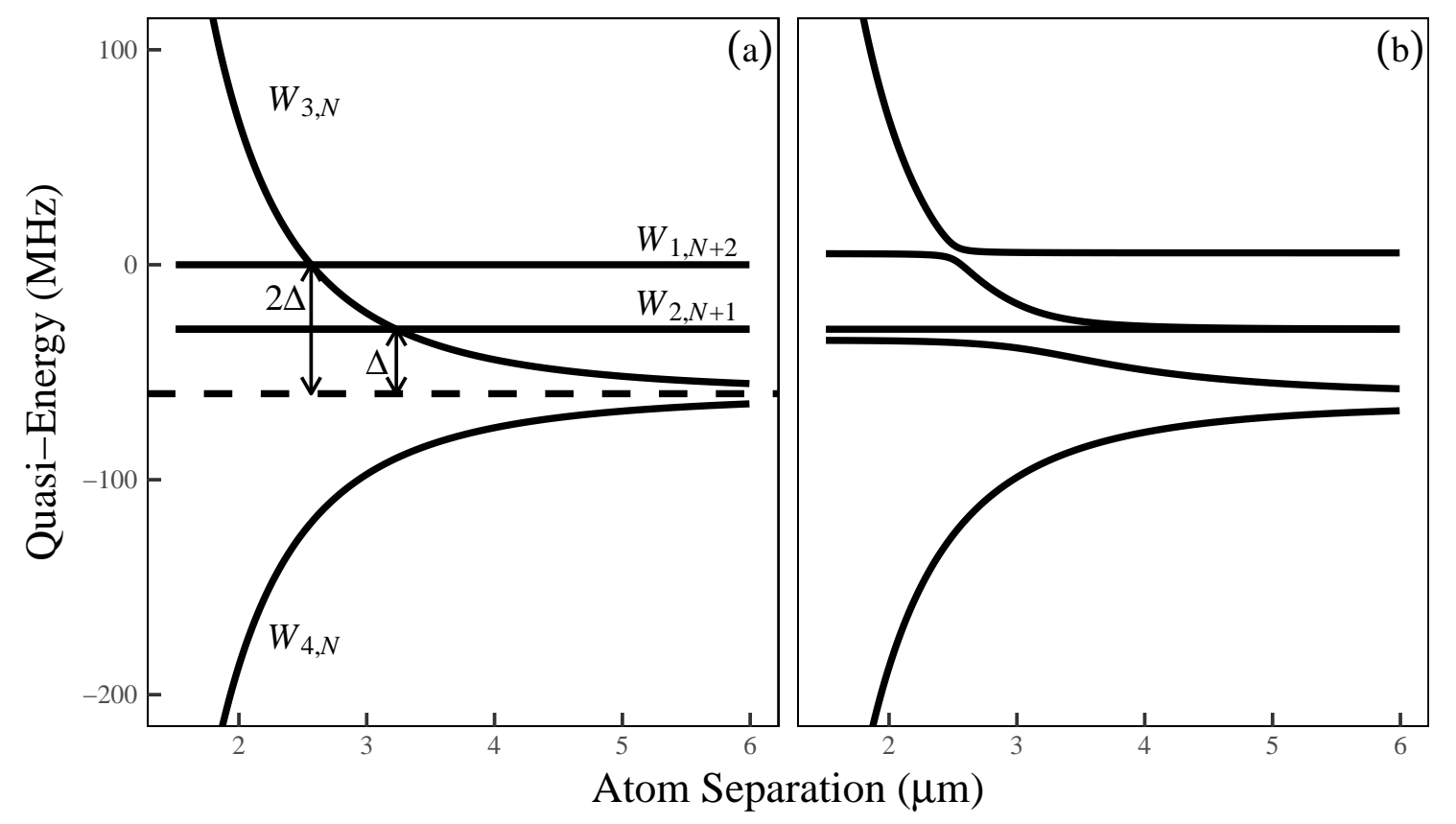

Figure 5.1: Energy of a laser dressed, DD resonant, atom pair with control laser detuning $\Delta=30 \mathrm{MHz}$ and single-atom Rabi frequency (a) $\Omega=0 \mathrm{MHz}$ and (b) $\Omega=19 \mathrm{MHz}$. In (a), $W_{1, N+2}$ is the energy of the $5 p_{3 / 2} 5 p_{3 / 2}$ trap state pair plus two blue-detuned photons. $W_{2, N+1}$ is the energy of the singly-excited Rydberg state, $5 p_{3 / 2} 32 p_{3 / 2}$ or $32 p_{3 / 2} 5 p_{3 / 2}$ plus one blue-detuned photon. $W_{3, N}$ and $W_{4, N}$ are the doubly-excited Rydberg states, $32 p_{3 / 2} 32 p_{3 / 2}+$ $32 s 33 s$ and $32 p_{3 / 2} 32 p_{3 / 2}-32 s 33 s$, respectively, with no additional photons. The energy $W_{1, N+2}$ is defined as 0 . The dashed horizontal line is the energy of the doubly-excited Rydberg state for atoms with infinite separation. In (b), the energies have developed laserinduced avoided crossings where the doubly-excited Rydberg state energy with dipoledipole interaction crosses with the other energy levels. The remaining flat energy level is the $5 p_{3 / 2} 32 p_{3 / 2}-32 p_{3 / 2} 5 p_{3 / 2}$ "dark" state that does not couple to the rest of the system. See Figure 5.2 for the composition of the states with laser interaction shown in (b). 


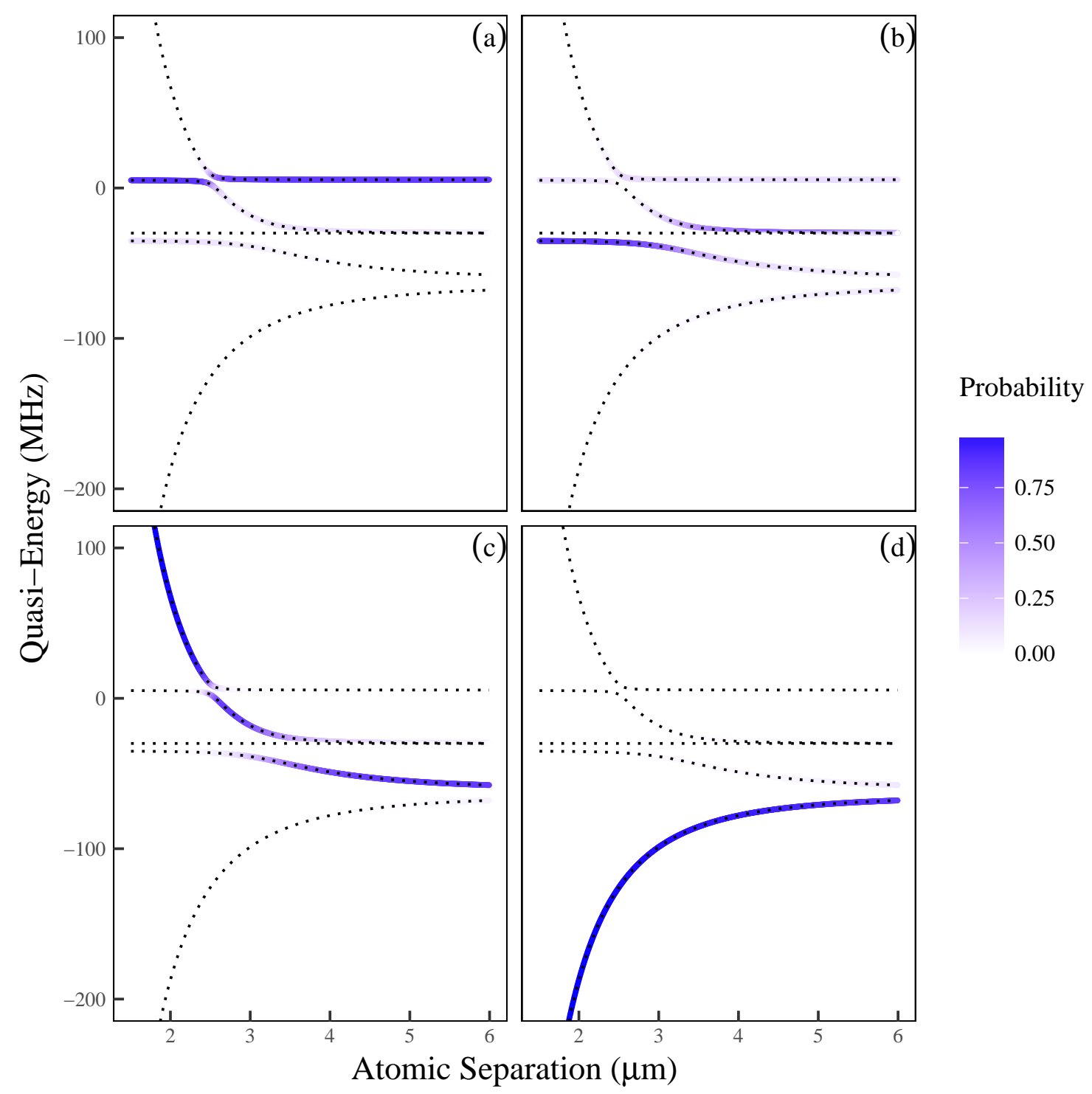

Figure 5.2: Energy of a laser dressed, DD resonant, atom pair for control laser detuning $\Delta=30 \mathrm{MHz}$ and Rabi frequency $\Omega=19 \mathrm{MHz}$, which allows for an adiabatic transition. The dashed lines are the energy levels as shown in Figure 5.1(b). The colors in each panel show the probability for the atom pairs to be in state (a) $5 p 5 p$, (b) $5 p 32 p+32 p 5 p$, (c) $32 p 32 p+32 s 33 s$, and (d) $32 p 32 p-32 s 33 s$. The $5 p 32 p-32 p 5 p$ state probability is not shown as it does not interact with the other states and resides entirely in the straight, horizontal dashed line. 
and rises quickly at small separations. With an atom-laser coupling of Rabi frequency $\Omega$, atom pairs on the upper energy curve are a separation-dependent mixture of the states $5 p_{3 / 2} 5 p_{3 / 2}, 5 p_{32} 32 p_{3 / 2}+32 p_{3 / 2} 5 p_{3 / 2}, 5 p_{32} 32 p_{3 / 2}-32 p_{3 / 2} 5 p_{3 / 2}, 32 p_{3 / 2} 32 p_{3 / 2}+32 s 33 s$, and $32 p_{3 / 2} 32 p_{3 / 2}-32 s 33 s$. Figure 5.2 gives the calculated probabilities for the atom pairs to be in a particular state as a function of separation. For the upper curve, atoms at large separations are mostly in the $5 p_{3 / 2} 5 p_{3 / 2}$ trap state with a small portion in the singly-excited Rydberg state $5 p_{3 / 2} 32 p_{3 / 2}+32 p_{3 / 2} 5 p_{3 / 2}$. At small separations, the atom pairs are entirely in the doubly-excited Rydberg state $32 p_{3 / 2} 32 p_{3 / 2}+32 s 33 s$. Let us assume we have a pair of atoms each initially in the $5 p_{3 / 2}$ trap state, at a large separation such that they lie on the upper energy curve. If the atoms move closer together, they reach the avoided crossing with the doubly-excited Rydberg state, bringing the two-photon transition into resonance. If the atoms are moving slowly enough, i.e., for sufficiently low temperatures $(\sim 0.2 \mathrm{~m} / \mathrm{s}$ for an atom pair at $\sim 70 \mu \mathrm{K})$, the atom pair can make an adiabatic transition to the doubly-excited Rydberg state, following the electronic energy curve. As the atom pair gains electronic energy the atoms lose kinetic energy, stop, and begin to move away from one another. As they move apart, they pass back through the avoided crossing, emit a pair of photons, and return to the $5 p_{3 / 2}$ state. The atoms only remain in the Rydberg state for a short time (a few $\mu \mathrm{s})$ while they are near one another [22]. The rest of the time, the atoms are cooled by the MOT while in the $5 p_{3 / 2}$ state and will not be transferred to untrapped states via spontaneous emission.

The preceding picture suggests that we have the ability to set the approximate minimum separation between atom pairs based on a transition to a repelling DD curve. The location of the avoided crossing between the $5 p_{3 / 2}$ trap state and the doubly-excited Rydberg state 
(the upper avoided crossing), and thus the approximate minimum separation, is set by the detuning of the control laser. The closer $\Delta$ is to 0 , the larger the minimum separation will be. The ability of atoms to move up the repelling DD energy curve is dependent on the relative speed of the atom pair, the strength of the DD interaction, and the control laser detuning and coupling strength. The atoms are continuously cooled by the MOT to keep their temperature low enough that they can adiabatically pass through the avoided crossing.

Note that for ${ }^{85} \mathrm{Rb}$, interactions of the form $n p n p \leftrightarrow n s n^{\prime} s$ are the most convenient, though they do require Stark mixing to allow for excitation from the $5 p_{3 / 2}$ state to the $n p$ Rydberg state. While an interaction of the form $n s n s \leftrightarrow n p n^{\prime} p$ would be preferable, such resonant interactions are hard to find due to the relative energy spacing of the $n p$ and $n s$ states.

Wall et al. suggested two possible experimental avenues to create this minimum separation control system. First, a control laser with large detuning $\Delta$ could be applied to an existing cold atom cloud. The control laser frequency would then be chirped to smaller detuning. This would create a force that initially pushes only the closest separated atoms apart and then moves to larger separations. The second implementation has the control laser at a fixed detuning $\Delta$ already incident on the interaction region while the MOT is forming. The control laser now acts to prevent atoms from approaching closer than the minimum separation while the MOT forms [22]. 


\subsection{Chirped Frequency Experiment}

In this section, we explore methods to control the minimum separation between atoms using a chirped frequency control laser. We describe how to implement the method, the measurements we made, and the complexities inherent to this system. Specifically, we discuss the limitations imposed by the density of the atoms and the tuning of the control laser.

\subsubsection{Experimental Procedure}

In this experiment, ${ }^{85} \mathrm{Rb}$ atoms at $\sim 70 \mu \mathrm{K}$ in the MOT described in Section 2.2 are Rydberg dressed from the $5 p_{3 / 2}$ trap state to the $32 p_{3 / 2}\left|m_{j}\right|=3 / 2$ Rydberg state by a control laser with detuning $\Delta$. The Rydberg dressing, as described in Section 5.1, uses the control laser and the $32 p_{3 / 2} 32 p_{3 / 2} \leftrightarrow 32 s 33 s$ coupling to put the atoms into a state where, at large separations, the atoms are a mixture of mostly $5 p 5 p$ and little to no $5 p 32 p+32 p 5 p$ pairs (see Figure 5.2(a) and (b)) and, at small separations, the atoms are entirely in the $32 p 32 p+32 s 33 s$ state (see Figure 5.2(c)). The $32 p_{3 / 2}$ state is accessible from the $5 p_{3 / 2}$ trap state by the application of the electric field that tunes the states to resonance, mixing a small amount of $n s$ and $n d$ character into the $p$-states. The atoms within the MOT are exposed to the control laser with some initial large detuning $\Delta_{I}$ that is then chirped (frequency shifted) to a smaller target detuning $\Delta_{T}$. The atoms are then allowed to interact for approximately $0.5 \mu$ s before being detected. State-selective field ionization (SSFI) is used to measure the population in the Rydberg states along with any stray ions that are produced.

To measure the effectiveness of this atom separation control method, we examine both 
the Rydberg population and the ion population within the MOT. Ions can be produced within a MOT prior to the application of a field ionization pulse due to collisions between Rydberg atoms $[131,143]$. Due to the long times used in these measurements, there are many opportunities for pairs of Rydberg atoms to collide. We can measure the effectiveness of our atom separation control method by examining the ion population relative to the Rydberg population. With no DD interaction, $5 p_{3 / 2}$ atoms with a small, singly-excited Rydberg state character will be able to closely approach one another and, potentially, ionize. With the DD coupling, the $5 p_{3 / 2}$ atoms will approach one another and be reflected at the minimum separation, preventing ionization. Thus, observation of a reduced ion population relative to the Rydberg population, with strong as compared to weak DD coupling, could serve as a signature of atom separation control.

The control laser is a $482 \mathrm{~nm}$ continuous wave (cw) laser that is frequency shifted by a double-pass AOM (described in Section 2.1.1) to ensure that the frequency shift does not deflect the laser. After the AOM, the power of the laser is approximately $50 \mathrm{~mW}$. The $482 \mathrm{~nm}$ laser is focused down to a spot size of approximately $0.1 \mathrm{~mm}$ by a $f=350 \mathrm{~mm}$ converging lens and is introduced to the MOT using the same geometry employed in the experiments in Chapters 3 and 4 . This gives a $5 p_{3 / 2} \rightarrow 32 p_{3 / 2}$ laser-atom coupling with a Rabi frequency of approximately $4 \mathrm{MHz}$ at the laser focus in the MOT. The target detuning $\Delta_{T}$ of the laser is set using a piezoelectric that adjusts the length of a FPI (see Section 2.3.5). The initial detuning $\Delta_{I}$ and subsequent chirp of the control laser frequency from the AOM is controlled by shifting the frequency of the RF field produced by the AOM driver. Unfortunately, due to the efficiency of the AOM, we can only shift the frequency of the control laser by $\sim 40 \mathrm{MHz}$ while maintaining sufficient laser power. All of the 
measurements are begun at the initial detuning and the frequency is tuned discretely in steps of $\delta$. There is a $10 \mathrm{~ms}$ delay between each step imposed by the speed of the AOM driver computer connection. The results shown in Section 5.2.2 are for a target detuning of $\Delta_{T}=$ $30 \mathrm{MHz}$, initial detunings $\Delta_{I}=30 \mathrm{MHz}, 40 \mathrm{MHz}, 50 \mathrm{MHz}, 60 \mathrm{MHz}$, and $70 \mathrm{MHz}$, and step sizes $\delta=1 \mathrm{MHz}$ and $5 \mathrm{MHz}$. This gives total times spent during the frequency chirp of $400 \mathrm{~ms}$ for the largest sweep to $20 \mathrm{~ms}$ for the shortest sweep. No frequency chirp occurs for $\Delta_{I}=30 \mathrm{MHz}$.

Laser excitation was performed at two total electric fields, $F=12.6 \mathrm{~V} / \mathrm{cm}$ and 18.6 $\mathrm{V} / \mathrm{cm}$ for on-DD resonance and off-DD resonance excitation, respectively. The on-DD resonance case should give the Rydberg dressing as shown in Figure 5.1(b). The off-DD resonance case should prevent the creation of a repelling DD curve and thus result in no atom separation control. All of the fields applied to the interaction region come via voltages applied to the field rods, as described in Chapter 2. At the end of the frequency sweeps, a high-voltage ramp is applied to ionize any Rydberg atoms and accelerate them to a multichannel plate (MCP) detector. Two main signals, the Rydberg atom population and the ion population, are measured by putting time gates around the signals in the MCP trace and integrating the signals within them (see Figure 5.3). Unlike in Chapters 3 and 4, it is more difficult for us to distinguish between the $32 p$ state and the $33 s$ state, so we integrate over the total Rydberg signal. An offset field of $2.758 \pm 0.005 \mathrm{~V} / \mathrm{cm}$ as described in Chapters 3 and 4 is added to the applied field to produce the total field in the interaction region. 


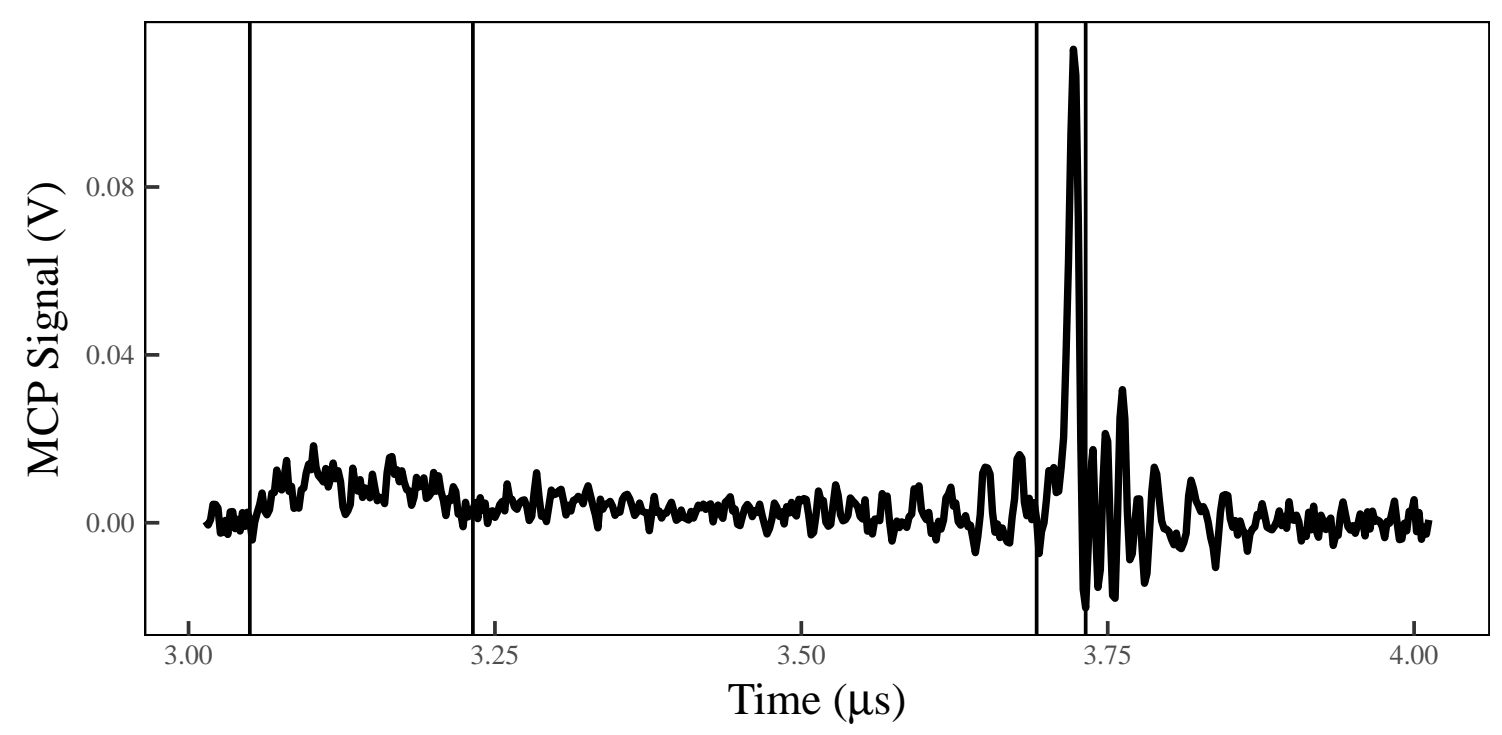

Figure 5.3: A sample field ionization trace for the fixed frequency control laser described in Section 5.3.1 with detuning $\Delta=15 \mathrm{MHz}$, MOT formation time $t_{\text {on }}=0.25 \mathrm{~s}$, and an electric field of $F=18.6 \mathrm{~V} / \mathrm{cm}$. The vertical lines on the right show the gate for the Rydberg states and the vertical lines on the left show the gate for the additional ions. The time $t=0$ is set to the arrival of the field ionization trigger. 


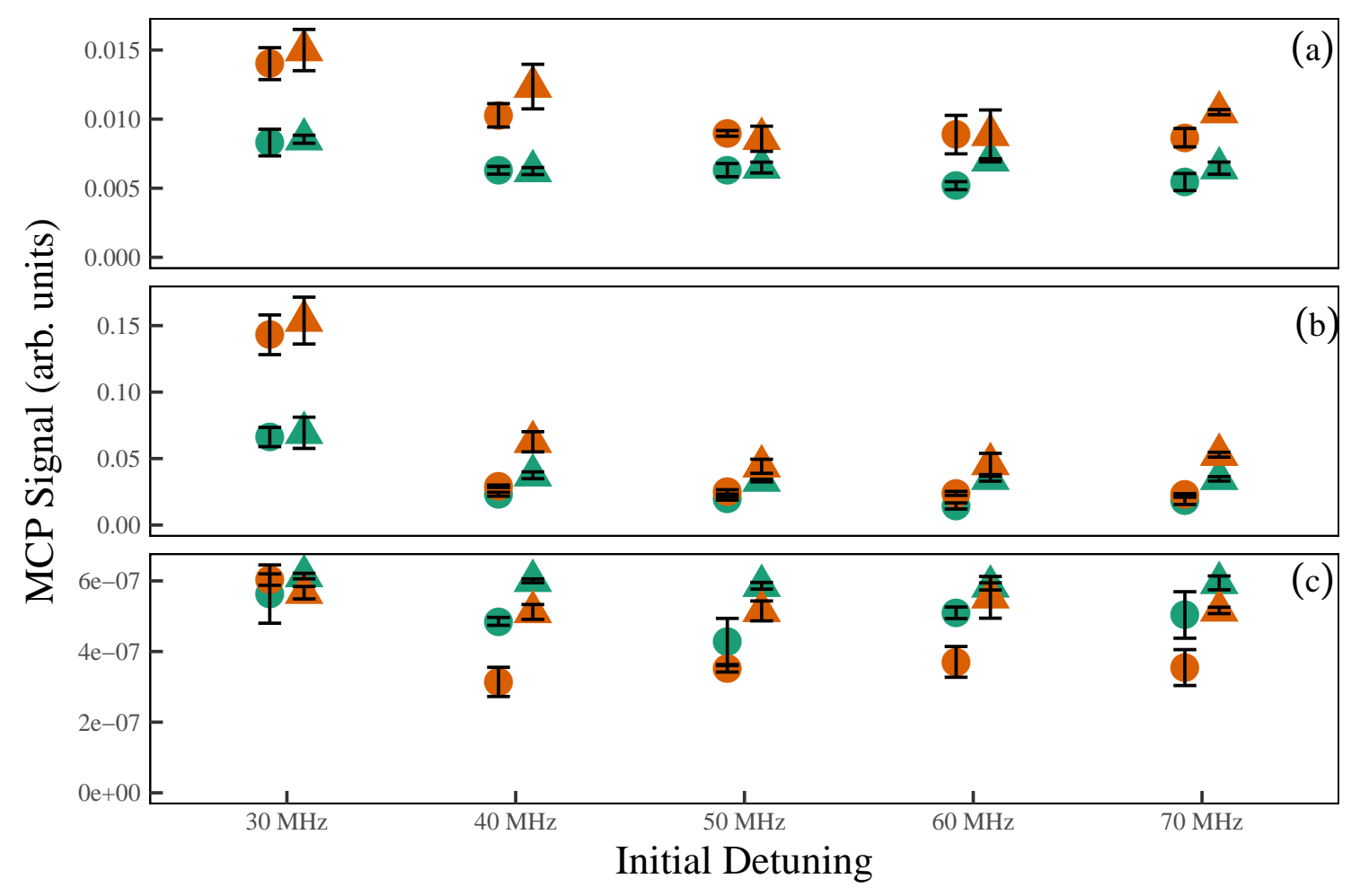

Figure 5.4: (a) Total Rydberg signal, (b) ion signal, and (c) MOT fluorescence as a function of initial detuning $\Delta_{I}$. The target detuning $\Delta_{T}=30 \mathrm{MHz}$ in all cases. The filled circles are for step sizes of $1 \mathrm{MHz}$ and the filled triangles are for step sizes of $5 \mathrm{MHz}$. The green points are for an applied electric field of $12.6 \mathrm{~V} / \mathrm{cm}$ (on resonance) and the orange points are for an applied electric field of $18.6 \mathrm{~V} / \mathrm{cm}$ (off resonance). Detection of the Rydberg atoms and ions was done $2 \mathrm{~ms}$ after the end of the frequency sweep (with the control laser still on). The Rydberg and ion signals are both normalized by the MOT fluorescence. No frequency chirp occurs for $\Delta_{I}=30 \mathrm{MHz}$. 


\subsubsection{Results and Analysis}

The results for the chirped-frequency minimum atom separation control experiment are shown in Figure 5.4. Overall, the Rydberg and ion signals for $\Delta_{I}=30 \mathrm{MHz}$, where no frequency chirp occurs, are larger than for the other initial detunings. Additionally, the Rydberg and ion signals are larger for the off-DD-resonance case while the signals are smaller for the on-DD-resonance case. We expect that, on-DD resonance, the majority of the atoms will be in the $5 p_{3 / 2}$ trap state with some small amount in the singly-excited Rydberg state $5 p 32 p+32 p 5 p$ (see Figure 5.2) and any ions created by collisions of closely spaced Rydberg excitations will be reduced. For the off-DD resonance case, we expect a larger number of ions due to collisions between Rydberg excitations [131, 143].

The measurements shown in Figure 5.4 show a general reduction in the ion population for on-DD resonance in agreement with our expectations and also shows a reduction in the Rydberg population that we must investigate further. To better interpret our results, we ran tests on the effect of changes in the laser-atom coupling strength as a function of applied electric field. We also studied the effect of laser pulse length on the Rydberg and ion signals as a function of different fixed control laser detunings and applied electric fields.

To determine the source of the differences in signals at different applied electric fields in Figure 5.4, we measured the coupling to the $32 p_{3 / 2}$ state for total electric fields of $F=$ $12.6 \mathrm{~V} / \mathrm{cm}$ (on-resonance) and $18.6 \mathrm{~V} / \mathrm{cm}$ (off-resonance). This was done by measuring the total Rydberg excitation as a function of the Rydberg excitation laser power at a fixed detuning $\Delta=30 \mathrm{MHz}$ relative to the Stark shifted Rydberg resonance at each total field. The power of the excitation laser was measured with the laser running in a cw mode and the laser was then chopped to a pulse length of $2 \mu$ s by the double-pass AOM described in 


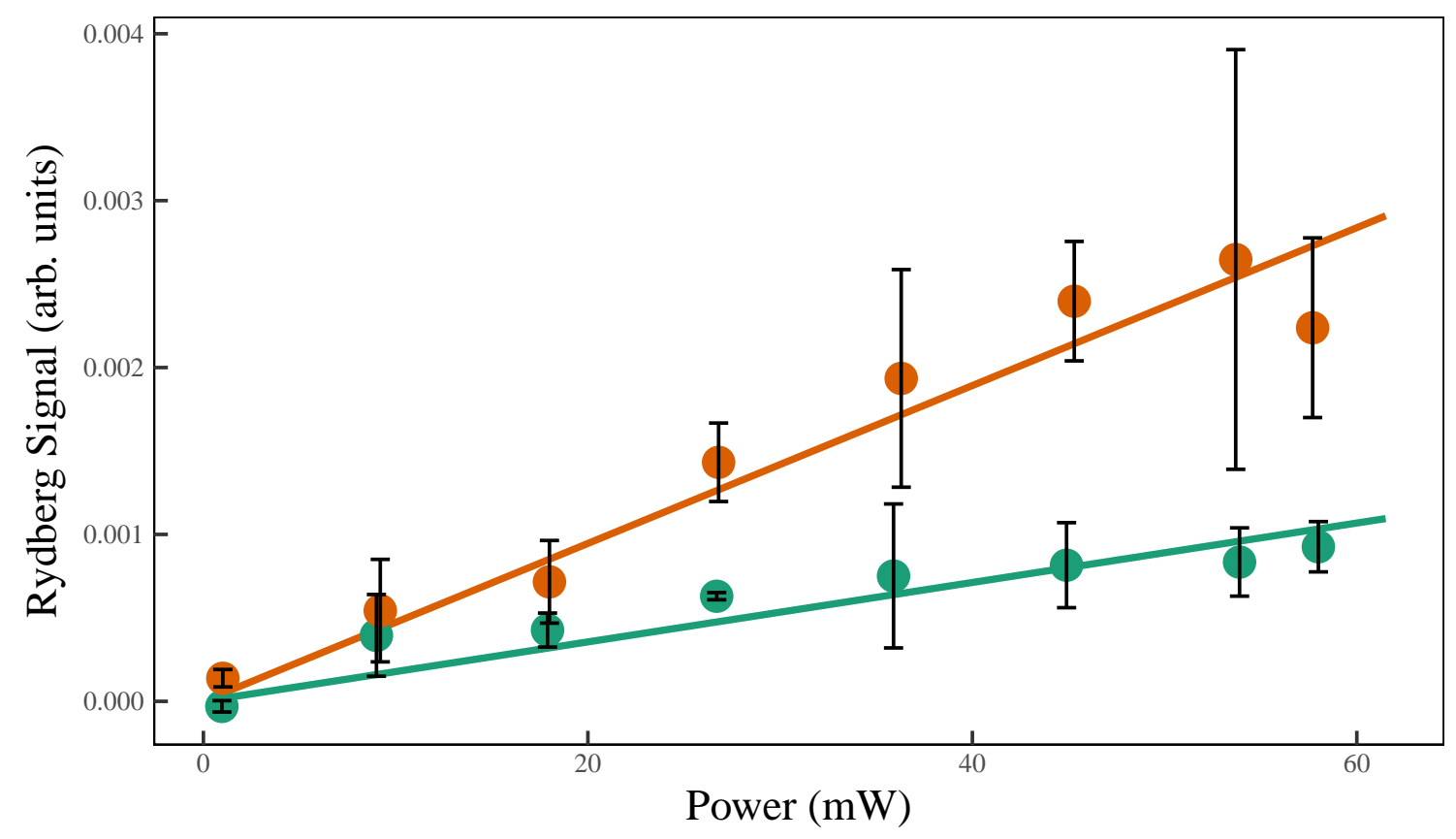

Figure 5.5: The total Rydberg signal as a function of Rydberg excitation laser power at a fixed excitation laser detuning of $\Delta=30 \mathrm{MHz}$. A $2 \mu$ s AOM chopped pulse was used for the excitation.The green points were taken in a total electric field of $12.6 \mathrm{~V} / \mathrm{cm}$ and the orange points were taken in a total field of $18.6 \mathrm{~V} / \mathrm{cm}$. The lines are linear fits with their y-intercept set to 0 . The Rydberg signal is normalized by the MOT fluorescence. The ratio between the two slopes $m_{18.6} / m_{12.6} \sim 2$.

Section 5.2.1. The relative coupling was determined by looking at the ratio of the slopes of a linear fit applied to the data (see Figure 5.5). The ratio of the slopes gave $m_{18.6} / m_{12.6} \sim$ 2.

Based on Stark calculations, we expect an increased Stark mixing of $n s$ and $n d$ character into the $32 p_{3 / 2}$ state to result in approximately a factor of 2 greater Rydberg signal for an off-DD resonance field of $F=18.6 \mathrm{~V} / \mathrm{cm}$ compared to the on-DD resonance field of 12.6 $\mathrm{V} / \mathrm{cm}$. As the field is increased, nearby $n s$ and $n d$ states are more strongly mixed into the $32 p_{3 / 2}$ state, giving a greater coupling of the weakly-mixed $5 p_{3 / 2} \leftrightarrow 32 p_{3 / 2}$ transition. 
We observe approximately this behavior in Figure 5.4 for small initial detunings, while we observe a smaller increase in Rydberg population at large detunings. A major difference between the small and large detuning measurements is the increased time that the atoms are exposed to the control laser. To determine the source of the decreased Rydberg and ion populations at off-DD resonance electric fields, we examined the populations as a function of control laser pulse length at fixed detuning.

We examined the effect of control laser pulse length by measuring the Rydberg and ion signals as a function of Rydberg excitation laser pulse length while fixing the detuning $\Delta$ of the excitation laser and the applied electric field. The detuning was adjusted using the piezoelectric of the Fabry-Pérot interferometer and was set to $\Delta=20 \mathrm{MHz}, 30 \mathrm{MHz}$, and $40 \mathrm{MHz}$. The total electric field was set to $F=12.6 \mathrm{~V} / \mathrm{cm}$ (on-DD-resonance), $14.7 \mathrm{~V} / \mathrm{cm}$, 18.6 V/cm, and $22.6 \mathrm{~V} / \mathrm{cm}$. The laser pulse was created by chopping a pulse of variable length $\tau$ off of the cw control laser. Ionization of the atoms was performed at the end of the laser pulse.

The results from these measurements can be seen in Figures 5.6 and 5.7. In Figure 5.6, the total Rydberg signal shows a peak for times $\tau \leq 0.5 \mathrm{~ms}$ and then drops to a constant value for times $\tau \geq 5 \mathrm{~ms}$. This drop in the Rydberg and ion populations is consistent with the drop observed in the chirped-frequency measurements at larger detunings. Our measurement of the Rydberg coupling accounts for the factor of approximately 2 increase in the Rydberg population at small initial detuning and the laser pulse length measurement accounts for the decrease in the Rydberg and ion populations at large initial detunings and, thus, longer times. This indicates that no atom separation control has occurred.

We now want to investigate the possible reasons why we are unable to observe atom 


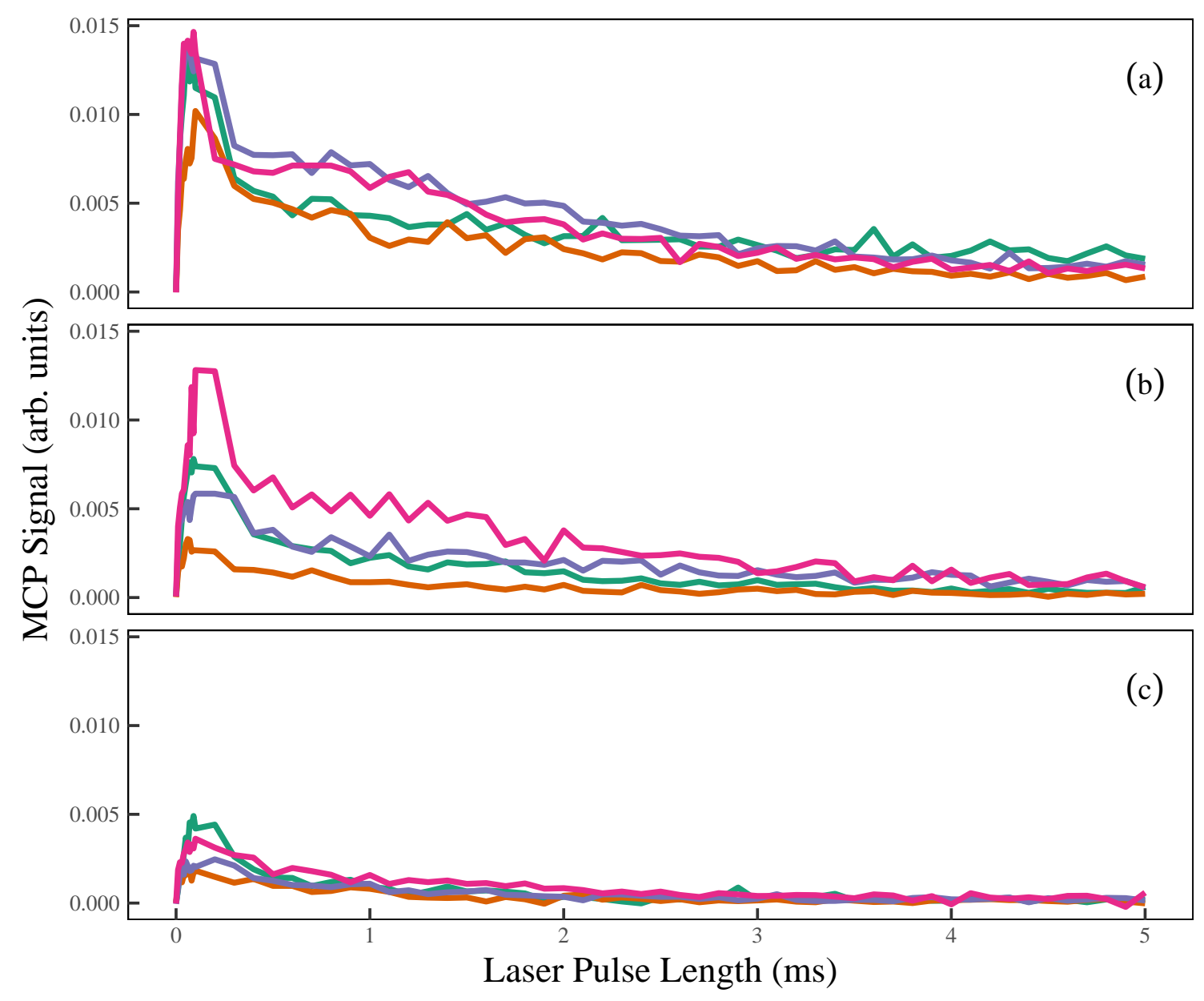

Figure 5.6: Total Rydberg signal as a function of Rydberg excitation laser pulse length for detuning $\Delta=$ (a) $20 \mathrm{MHz}$, (b) $30 \mathrm{MHz}$, and (c) $40 \mathrm{MHz}$. The different solid lines are for excitation at electric fields of $12.6 \mathrm{~V} / \mathrm{cm}$ (green), $14.7 \mathrm{~V} / \mathrm{cm}$ (orange), $18.6 \mathrm{~V} / \mathrm{cm}$ (purple), and $22.6 \mathrm{~V} / \mathrm{cm}$ (pink). See Figure 5.7 for total ion signals from the same measurement. The Rydberg signals have been normalized by the MOT fluorescence. 


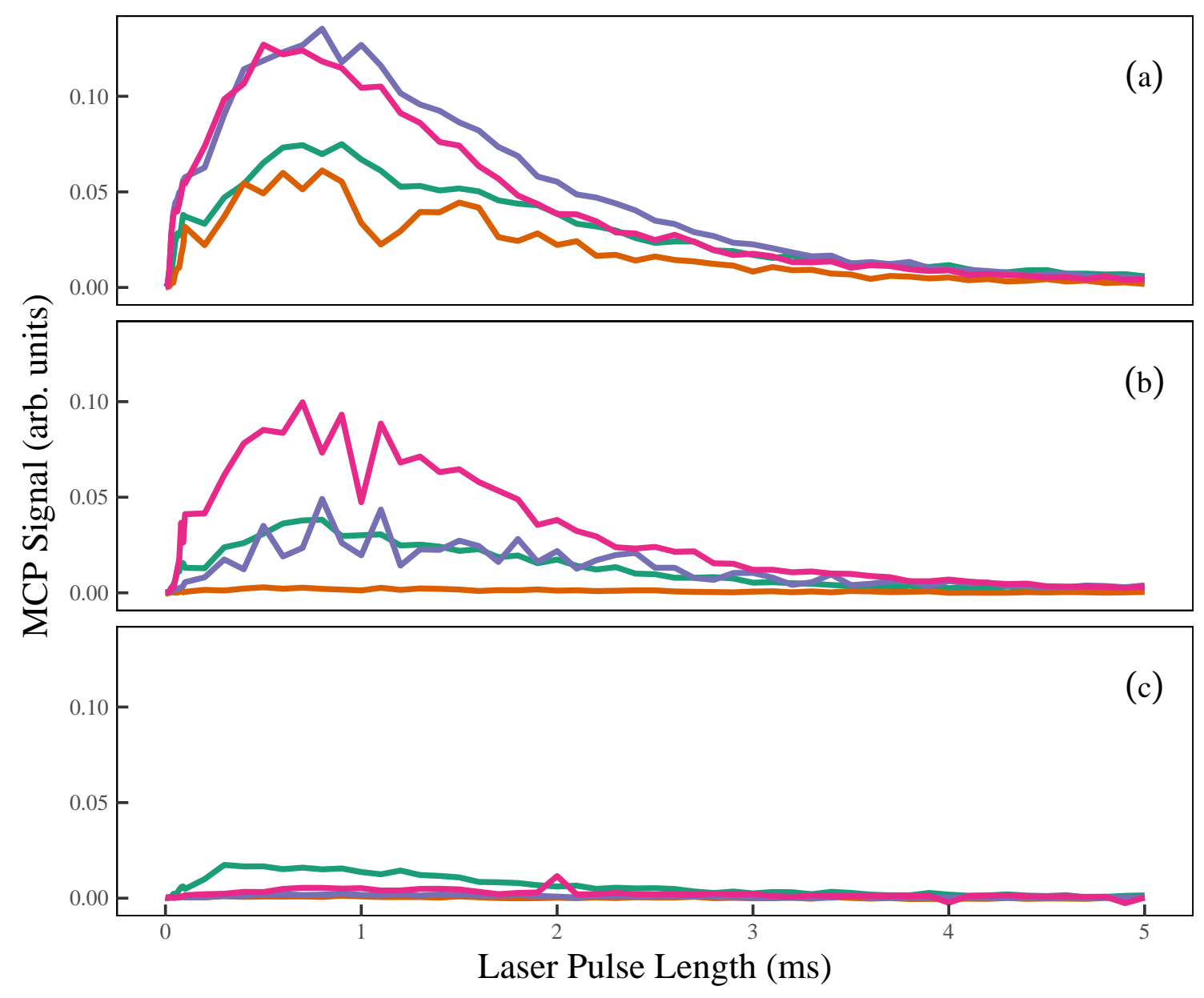

Figure 5.7: Total ion signal as a function of Rydberg excitation laser pulse length for detuning $\Delta=$ (a) $20 \mathrm{MHz}$, (b) $30 \mathrm{MHz}$, and (c) $40 \mathrm{MHz}$. The different solid lines are for excitation at electric fields of $12.6 \mathrm{~V} / \mathrm{cm}$ (green), $14.7 \mathrm{~V} / \mathrm{cm}$ (orange), $18.6 \mathrm{~V} / \mathrm{cm}$ (purple), and $22.6 \mathrm{~V} / \mathrm{cm}$ (pink). See Figure 5.6 for total Rydberg signals from the same measurement. The ion signals have been normalized by the MOT fluorescence. 
separation control. In the laser pulse length measurements described above, we found that as the laser detuning became larger the measurements made at lower field had higher maximum signals compared to the measurements made at high field. For example, in the laser pulse length measurement for a $\Delta$ of $40 \mathrm{MHz}$ shown in Figure $5.6(\mathrm{c})$ the $12.6 \mathrm{~V} / \mathrm{cm}$ measurement has the largest Rydberg signal. A similar phenomenon is seen in the ion signal with the peak occurring at times $\tau \approx 2 \mathrm{~ms}$ and also approaching constant values by $\tau=$ $5 \mathrm{~ms}$. From this data alone, it is unclear why the on-DD-resonance excitation dominates the Rydberg and ion signals at large $\Delta$. To this end, spectroscopy of the $32 p_{3 / 2}\left|m_{j}\right|=3 / 2$ state was performed to search for any spectral features that might be present at the laser detunings and electric fields used.

Spectroscopy is performed by sweeping the frequency of the Rydberg excitation laser using the AOM that also chops the cw laser into a $2 \mu$ s pulse. The frequency is swept around a center point $30 \mathrm{MHz}$ detuned relative to the Stark-shifted $32 p_{3 / 2}\left|m_{j}\right|=3 / 2$ Rydberg resonance due to the limited sweeping range of the AOM. The applied electric field is varied to give us the spectroscopy as a function of electric field. A sample of the results from the spectroscopy can be seen in Figure 5.8. In each of these sweeps, the left-most peak is the $32 p_{3 / 2}\left|m_{j}\right|=3 / 2$ Rydberg resonance and the right-most peak is a satellite peak that shifts in frequency as a function of electric field. The satellite peak is a feature that we do not expect to see on the high frequency side of the $32 p_{3 / 2}\left|m_{j}\right|=3 / 2$ Rydberg resonance and may be responsible for the larger Rydberg resonances observed for the on-DD resonance field laser pulse length measurements.

A map of the $32 p_{3 / 2}\left|m_{j}\right|=3 / 2$ Rydberg resonance and satellite peak locations can be seen in Figure 5.9. Figure 5.9 gives a comparison between the peak locations and the 


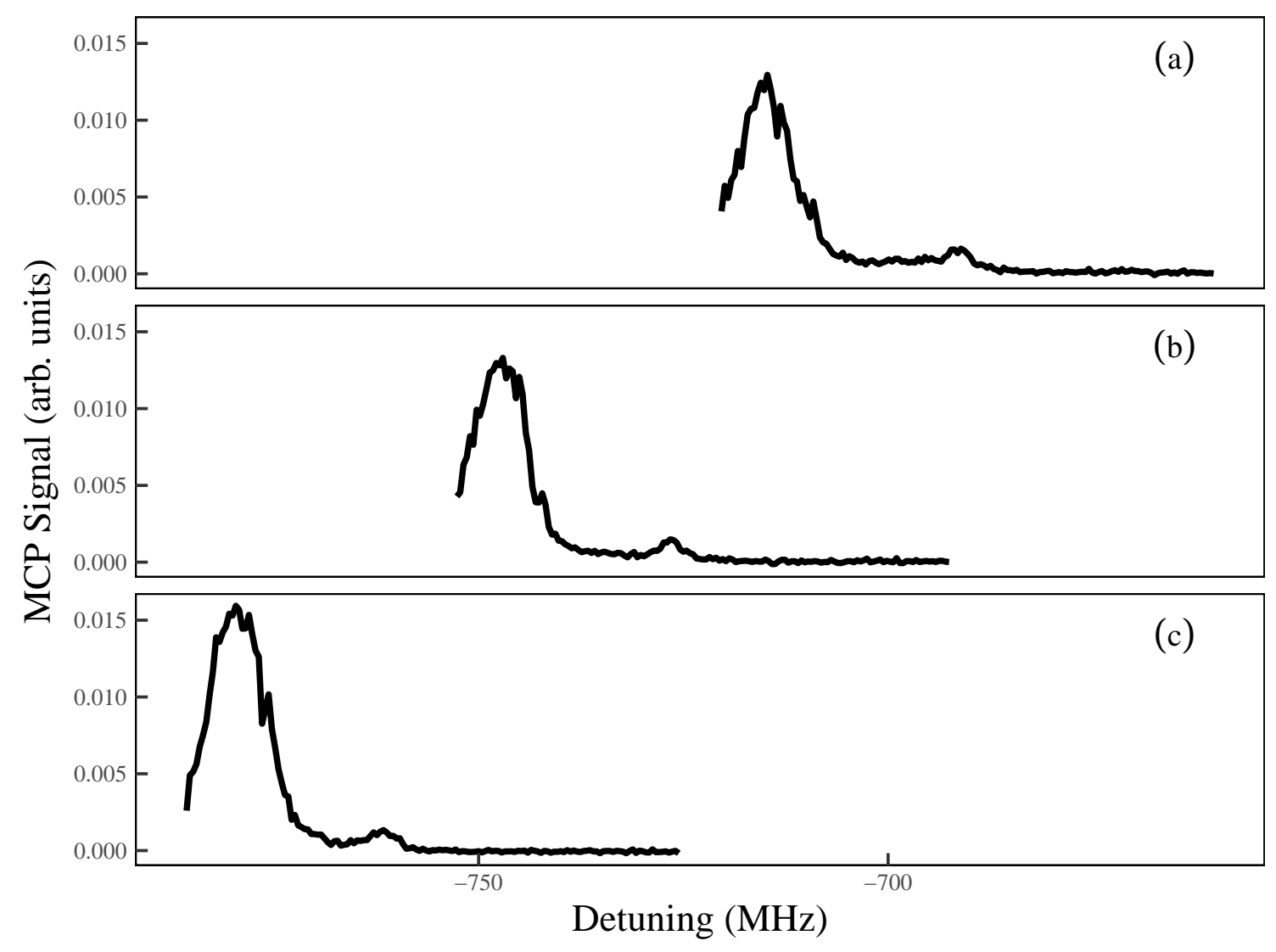

Figure 5.8: Rydberg signal as a function of detuning from the zero-field $32 p_{3 / 2}$ excitation frequency with an applied electric field of (a) $12.4 \mathrm{~V} / \mathrm{cm}$, (b) $12.6 \mathrm{~V} / \mathrm{cm}$, and (c) $13.0 \mathrm{~V} / \mathrm{cm}$. The large peak seen at lower frequencies is the $32 p_{3 / 2}\left|m_{j}\right|=3 / 2$ Rydberg state. The small peak seen at higher frequencies is a satellite peak identified as transitions to the $32 p_{3 / 2}$ $\left|m_{j}\right|=1 / 2$ Rydberg state from the lower-lying hyperfine levels of the $5 p_{3 / 2}$ trap state. 
laser frequencies required to excite the $32 p_{3 / 2}\left|m_{j}\right|=1 / 2$ and $3 / 2$ states from the various hyperfine split $5 p_{3 / 2}$ trap states. Typically, we assume that only the $\mathrm{F}=4$ hyperfine state of $5 p_{3 / 2}$ is populated in the MOT with some small amount entering the $\mathrm{F}=3$ hyperfine state as well. This shows that, in addition to the small population in the $\mathrm{F}=3$ hyperfine state, there is also a small population in the $\mathrm{F}=2$ hyperfine state. The $5 p_{3 / 2} \mathrm{~F}=2$ state could be populated due to either off-resonant excitation from the $5 s_{1 / 2} \mathrm{~F}=3 \rightarrow 5 p_{3 / 2} \mathrm{~F}=4$ trapping transition or off-resonant excitation from the $5 s_{1 / 2} \mathrm{~F}=2 \rightarrow 5 p_{3 / 2} \mathrm{~F}=3$ re-pumping transition. These populations lead to a direct excitation to the $32 p_{3 / 2}\left|m_{j}\right|=1 / 2$ state that is intersecting with the $32 p_{3 / 2}\left|m_{j}\right|=3 / 2$ state. By shifting the data $-0.5 \mathrm{~V} / \mathrm{cm}$, the data lines up reasonably well with the calculated excitation frequencies. This suggests that the additional population observed for the $F=12.6 \mathrm{~V} / \mathrm{cm}$ laser pulse scans in Figures 5.6 and 5.7 is likely due to these additional, field dependent, resonances that occur near $F=12.6$ $\mathrm{V} / \mathrm{cm}$ and disappear at higher electric fields.

Our additional measurements show that we expect more Rydberg atoms at higher applied electric fields due to the increased Rydberg coupling. We also showed that there is a change in the Rydberg and ion signals for longer pulse times consistent with our chirped frequency measurement. This helps us conclude that the signal differences we see are unlikely to be due to atom separation control. Additionally, we have measured transitions that appear to the blue (higher frequency) of the $32 p_{3 / 2}\left|m_{j}\right|=3 / 2$ Rydberg state. These additional resonances may affect our ability to control atom separation by providing DD interactions that are red-detuned from the additional resonances for smaller blue detuning from the $32 p_{3 / 2}\left|m_{j}\right|=3 / 2$ Rydberg state. These red detuned interactions would be attractive and cause the atoms to accelerate towards one another instead of repelling. 


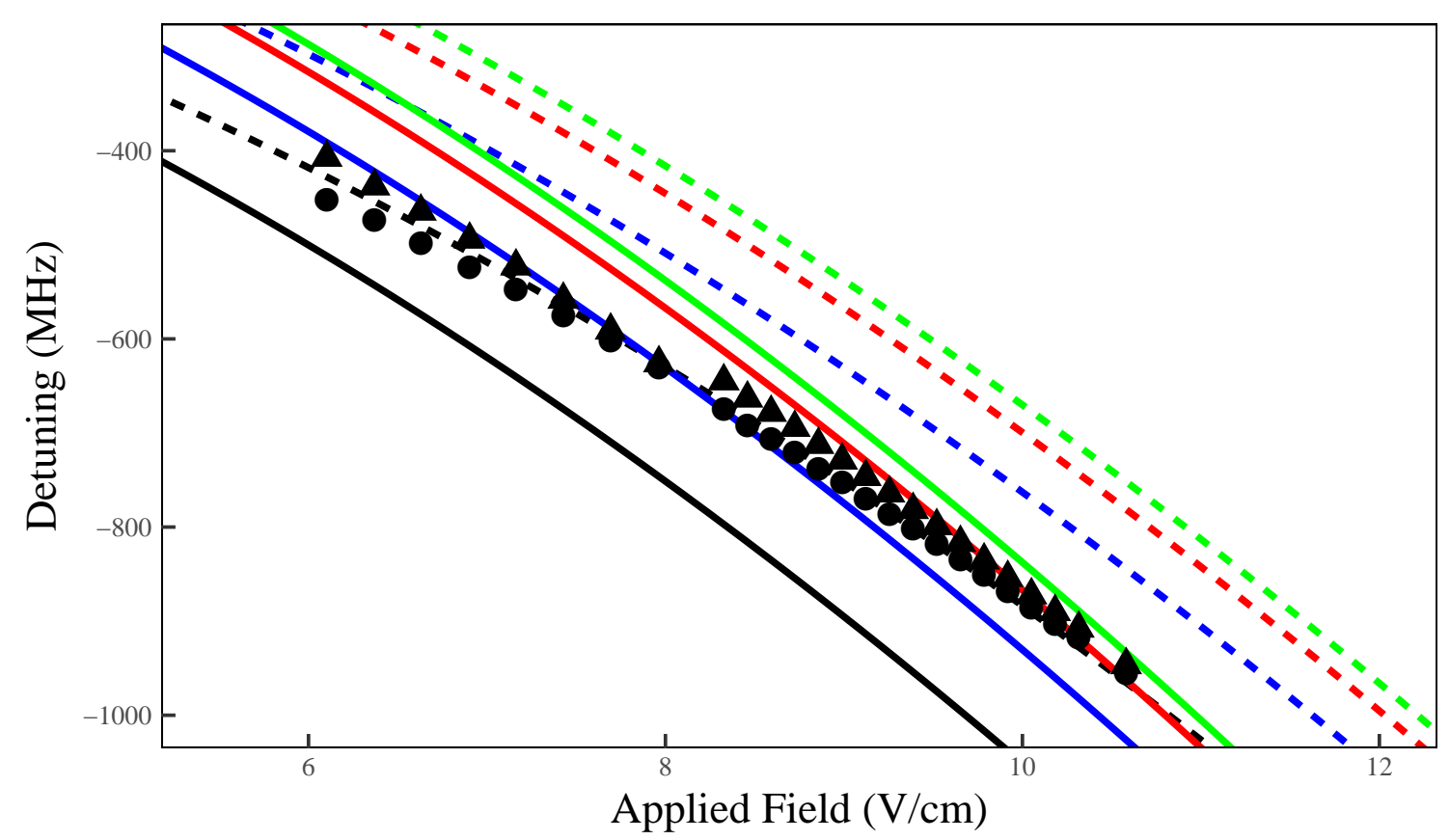

Figure 5.9: Detuning from the zero electric field Rydberg excitation laser frequency as a function of applied electric field. The points are the measured excitation frequencies for the large peak (filled circles) and small peak (filled triangles) from Figure 5.8. The lines are calculated excitation frequencies based on a Stark map calculation with the solid lines being excitation to the $32 p_{3 / 2}\left|m_{j}\right|=1 / 2$ state and dashed lines being excitation to the $32 p_{3 / 2}$ $\left|m_{j}\right|=3 / 2$ state. The colors are for excitation from the $5 p_{3 / 2}$ trap state with hyper-fine state $F=4$ (black), 3 (blue), 2 (red), and 1 (green). The measured points have been shifted by $-0.5 \mathrm{~V} / \mathrm{cm}$ to better align with the calculation. 
Another complication in this experiment is the limitation in how far we can detune the control laser. At $\Delta=30 \mathrm{MHz}$, the avoided crossing in Figure 5.1 is at $r \sim 2.5 \mu \mathrm{m}$. The maximum detuning we can reach with our current equipment is a detuning $\Delta=100 \mathrm{MHz}$, which has an avoided crossing at $r \sim 1.7 \mu \mathrm{m}$. As can be seen in Figure 5.10, $\sim 12 \%$ of the atom pairs are at separations less than or equal to $1.7 \mu \mathrm{m}$. This leaves a large number of atoms below the minimum separation. These can result in additional Rydberg excitation and ion formation. Ultimately, this will prevent us from truly controlling the minimum atom separation. To get all of the atoms in a state that would allow for optimum minimum separation control, the detuning of the laser would need to be at least $\Delta \geq 500 \mathrm{MHz}$ up to $\Delta \sim 5 \mathrm{GHz}$ for a minimum separation $r_{\min } \sim 1 \mu \mathrm{m}$ and $0.5 \mu \mathrm{m}$, respectively. These detunings are currently inaccessible by our equipment. Additionally, those large detunings may put the atoms in contact with additional resonances, as seen in Figure 5.9, that could shut off the atom control interaction.

\subsection{Fixed Frequency Experiment}

In this section we describe attempts to control the minimum separation of atoms using a fixed-frequency control laser. We discuss our implementation of the method, the measurements made using this technique, and possible future experiments.

\subsubsection{Experimental Procedure}

For this experiment, ${ }^{85} \mathrm{Rb}$ atoms in the $5 p_{3 / 2}$ trap state at $\sim 70 \mu \mathrm{K}$ in a MOT are Rydberg dressed with the $32 p_{3 / 2}\left|m_{j}\right|=3 / 2$ state under the same conditions as in Section 5.2.1 with 


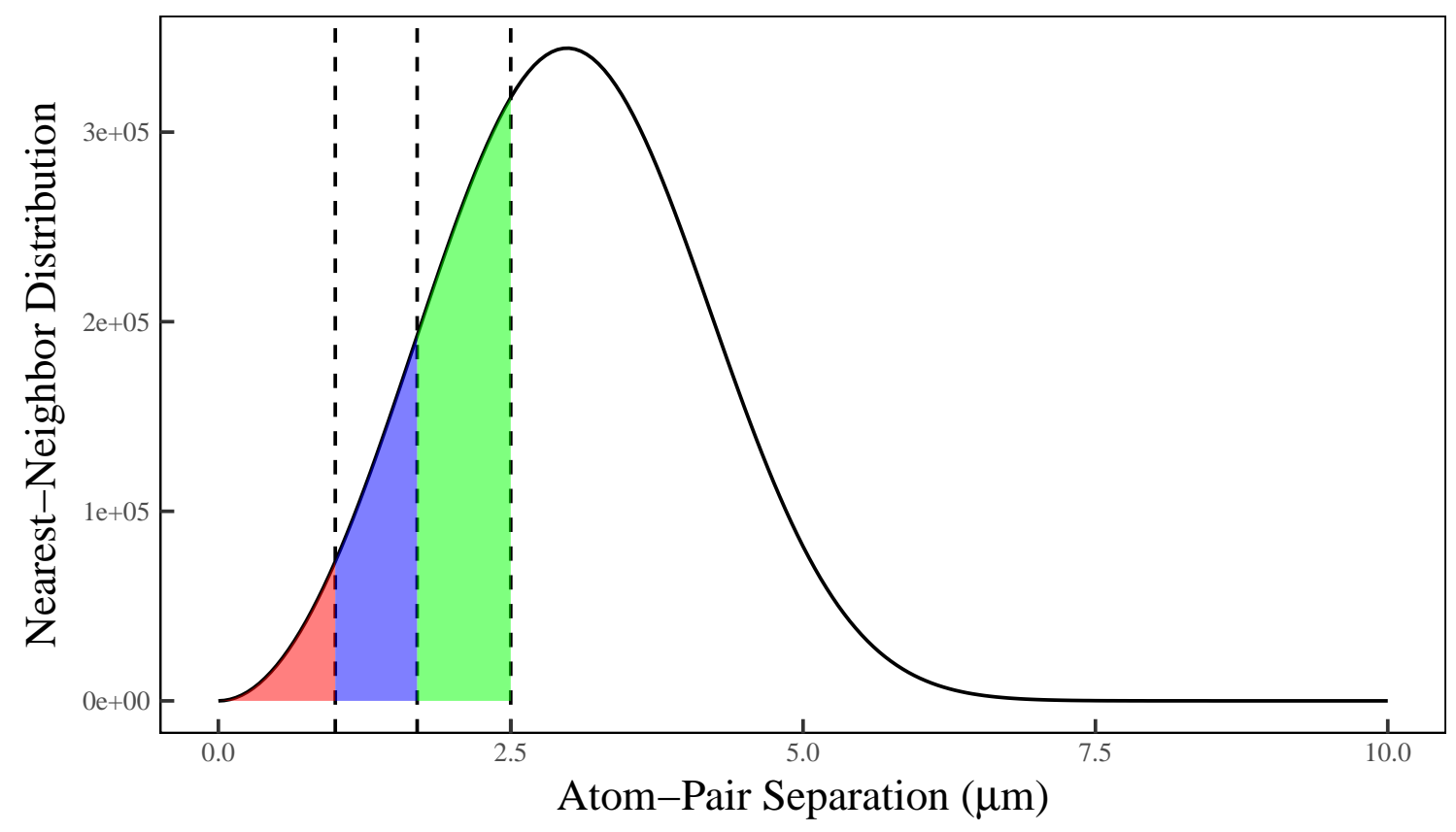

Figure 5.10: Nearest-neighbor probability distribution for a nominal density of $6 \times 10^{9}$ $\mathrm{cm}^{-3}$. The shaded sections are for separations of less than $1 \mu \mathrm{m}$ (furthest left and shaded red), $1.7 \mu \mathrm{m}$ (center and shaded blue), and $2.5 \mu \mathrm{m}$ (furthest rigth and shaded green). $2.7 \%$ of the atoms are in the red section, $12 \%$ are in the red and blue sections, and $33 \%$ are in all of the shaded sections.

the following changes. The re-pump laser is first turned off, allowing the MOT to dissipate. While the re-pump laser is turned off, the area where the MOT forms is then exposed to a control laser with a fixed detuning $\Delta$. The re-pump is switched on and the MOT is allowed to reform for a time $\tau$ while being illuminated by the control laser. State-selective field ionization (SSFI) is used to measure the population in the Rydberg state along with any indirectly produced ions. The control laser is a $482 \mathrm{~nm} \mathrm{cw}$ laser that is chopped by a double-pass AOM with a power of $50 \mathrm{~mW}$ after the AOM. The $482 \mathrm{~nm}$ laser is focused down to a spot size of approximately $0.1 \mathrm{~mm}$ by a $f=350 \mathrm{~mm}$ converging lens and is introduced 
to the MOT using the same geometry as the experiments in Chapters 3 and 4 . This gives a $5 p_{3 / 2} \rightarrow 32 p_{3 / 2}$ laser-atom coupling with a Rabi frequency of approximately $4 \mathrm{MHz}$ at the laser focus in the MOT. The detuning $\Delta$ of the control laser is set using a piezoelectric that adjusts the length of a FPI (see Section 2.3.5). The results shown in Section 5.3.2 are for control laser detuning $\Delta=15 \mathrm{MHz}, 30 \mathrm{MHz}$, and $40 \mathrm{MHz}$ relative to the Stark-shifted $5 p_{3 / 2} \rightarrow 32 p_{3 / 2}\left|m_{j}\right|=3 / 2$ Rydberg transition.

The re-pump laser is switched using an AOM in a single-pass configuration. When switched, the re-pump laser light is removed from the chamber. With the re-pump laser light removed, the MOT takes $\sim 1$ s to dissipate. This is measured by illuminating the MOT with an on-resonance, $482 \mathrm{~nm}, 3 \mu$ s long laser pulse for a variable time $t_{\text {off }}$ after the re-pump is switched and measuring the resulting Rydberg population. Figure 5.11 shows the results of measurements where the Rydberg population is measured out to turn-off times of $t_{\text {off }}=1.5 \mathrm{~s}$. The long amount of time for MOT dissipation is likely due to some small leakage of the re-pump laser light through the AOM.

The measurement of the MOT dissipation time was further confirmed by measuring how long it takes for the MOT to turn back on. If the MOT has not fully dissipated, Rydberg population is observed immediately after re-establishing the re-pump, indicative of an already present atom cloud. The MOT turn-on time was measured by exciting $32 p_{3 / 2}$ Rydberg states with a $3 \mu \mathrm{s}, 482 \mathrm{~nm}$ pulse a time $t_{\text {on }}$ after the re-pump was switched into the chamber, giving $\sim 2 \mathrm{~s}$ for the MOT to refill. For these measurements, and the subsequent fixed-frequency control laser measurements, the re-pump was turned off for $t_{o f f}=1 \mathrm{~s}$ prior to the introduction of the Rydberg excitation laser or control laser. We examined the atom separation control effects for times $t_{\text {on }}=50 \mathrm{~ms}, 100 \mathrm{~ms}, 150 \mathrm{~ms}, 200 \mathrm{~ms}$, and $250 \mathrm{~ms}$ 


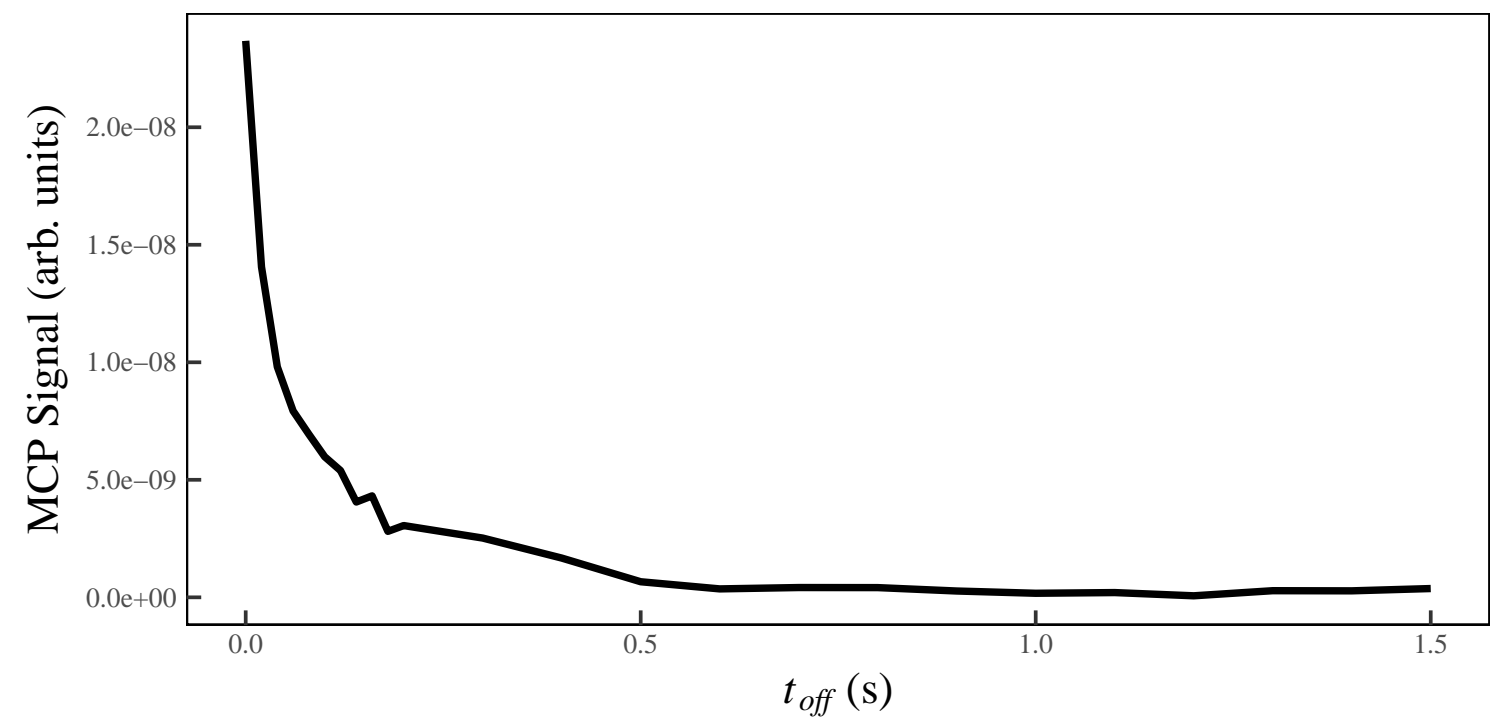

Figure 5.11: Rydberg atom population as a function of re-pump turn-off time $t_{\text {off }}$. Complete dissipation of the MOT is achieved after approximately $1 \mathrm{~s}$.

where the MOT has begun to fill and is reaching $~ 50 \%$ capacity.

As in Section 5.2.1, laser excitation was performed at two electric fields, $F=12.6$ $\mathrm{V} / \mathrm{cm}$ and $18.6 \mathrm{~V} / \mathrm{cm}$ for on-DD resonance and off-DD resonance excitation, respectively. The total electric field $F$ is the sum of the electric field applied to the rods and the offset field of $2.758 \pm 0.005 \mathrm{~V} / \mathrm{cm}$ as described in Chapters 3 and 4. Rydberg atom population and indirectly produced ions are accelerated towards the MCP detector by the application of a field ionization pulse. For these measurements, the time-dependent current from the MCP is recorded directly and saved for additional signal processing.

The output from the MCP is measured on an oscilloscope and recorded using the LabVIEW program described in Section 2.4.1. Field ionization traces from the oscilloscope are recorded for each shot of the laser. Fourier filtering is performed on the field ionization (FI) traces after data collection using the signal package [94] to remove background 
oscillations. Figure 5.3 shows a sample FI trace after Fourier filtering with $\Delta=15$ $\mathrm{MHz}, t_{o n}=0.25 \mathrm{~s}$, and $F=18.6 \mathrm{~V} / \mathrm{cm}$. FI trace gates are set such that integration occurs around the peaks representing the Rydberg population (rightmost vertical lines in Figure 5.3), the ion population (leftmost vertical lines in Figure 5.3), and the total measured excitation (the sum of both gates). The non-Rydberg, indirect ion population measured here is attributed to collisions between Rydberg atoms as described in Section 5.2.1. The results from integrating the Rydberg FI gate and the indirect ion population gate are divided by the total ion yield for normalization.

\subsubsection{Results and Analysis}

Figures 5.12, 5.13, and 5.14 show the normalized Rydberg and ion populations from the fixed-frequency, atom separation control measurements for $\Delta=15 \mathrm{MHz}, 30 \mathrm{MHz}$, and $40 \mathrm{MHz}$, respectively. As with the chirped frequency measurements, we expect that if the on-DD resonance conditions limit the minimum separation between atoms, then, relative to the off-resonance condition, a decrease in the ion population relative to the Rydberg population should be observed. Off-DD resonance, we expect to get more ions due to interactions between closely spaced Rydberg atoms.

Figure 5.12 shows Rydberg and ion population detected in approximately equal proportion for both on- and off-DD resonant fields. This implies no atom separation control. This is expected for a detuning of $\Delta=15 \mathrm{MHz}$ due to direct excitation to the $32 p_{3 / 2}$ $\left|m_{j}\right|=3 / 2$ state from the energetically broad $5 p_{3 / 2}$ trap state. Figures 5.13 and 5.14 show a decrease in the Rydberg population and an increase in the ion population at low $t_{o n}$ for the on-resonances vs. off-resonance case with $\Delta=30 \mathrm{MHz}$ and $40 \mathrm{MHz}$. This indicates some 


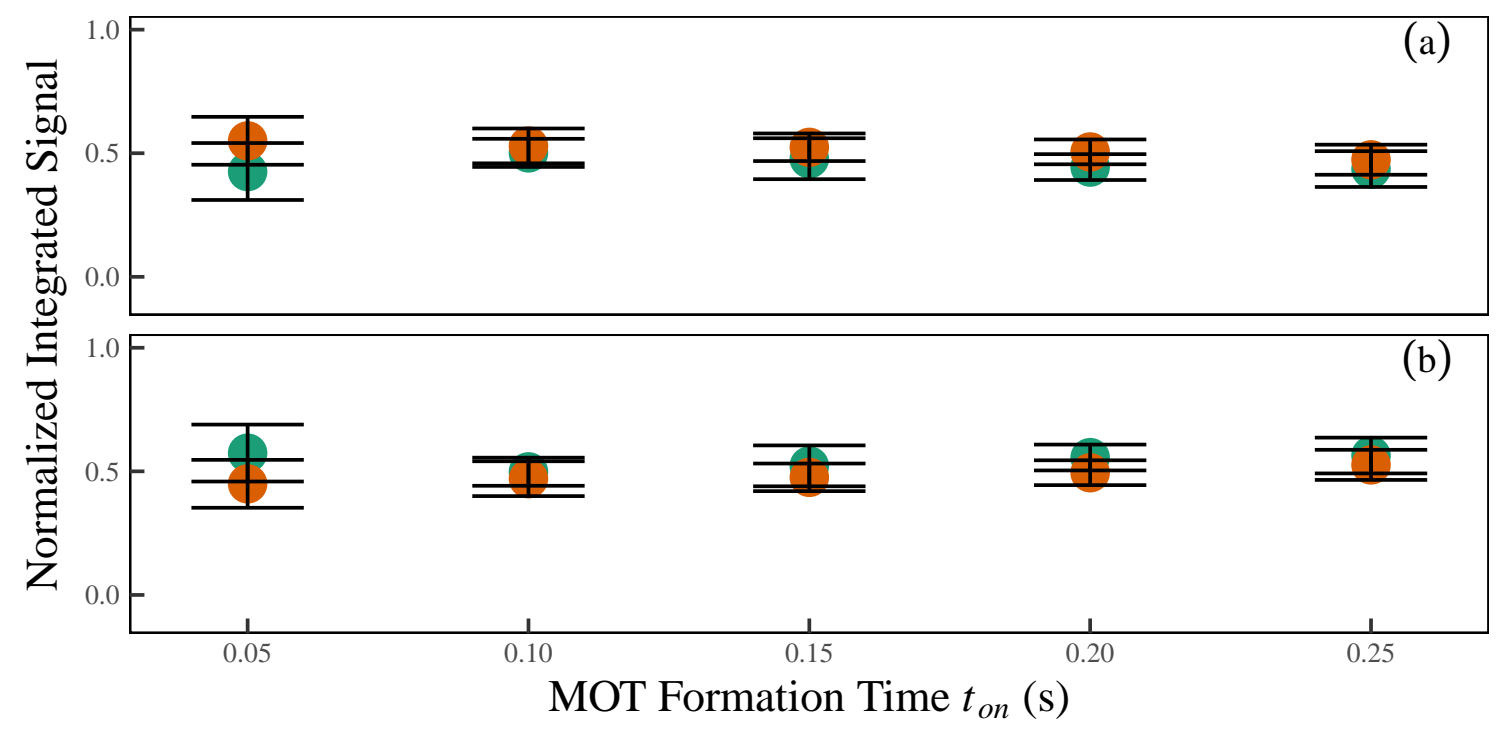

Figure 5.12: (a) Normalized Rydberg population and (b) normalized ion population for a control laser detuning $\Delta=15 \mathrm{MHz}$. Measurements were made at electric fields of $F=$ $12.6 \mathrm{~V} / \mathrm{cm}$ (green points) and $18.6 \mathrm{~V} / \mathrm{cm}$ (orange points). Both Rydberg and ion populations were normalized by dividing by the total ion yield (sum of the Rydberg and ion populations).

effect related to the presence of the on-DD resonance electric field. However, the errors bars are largely overlapping and it is difficult to determine whether it is an actual effect or purely noise due to the low signals at large $\Delta$.

While we might hope to see some signature of atom separation control at $\Delta=30$ $\mathrm{MHz}$ and $40 \mathrm{MHz}$, the control laser Rabi frequency used for both the chirped-frequency control laser and the fixed-frequency control laser is likely not high enough to make the atom separation control interaction purely adiabatic. We can estimate the probability that the atoms traverse the avoided crossing adiabatically by

$$
P_{\text {adiabatic }}=1-\exp \left[-\frac{\pi \Omega^{4}(C / 2)^{1 / 3}}{24 \Delta^{10 / 3} v}\right]
$$




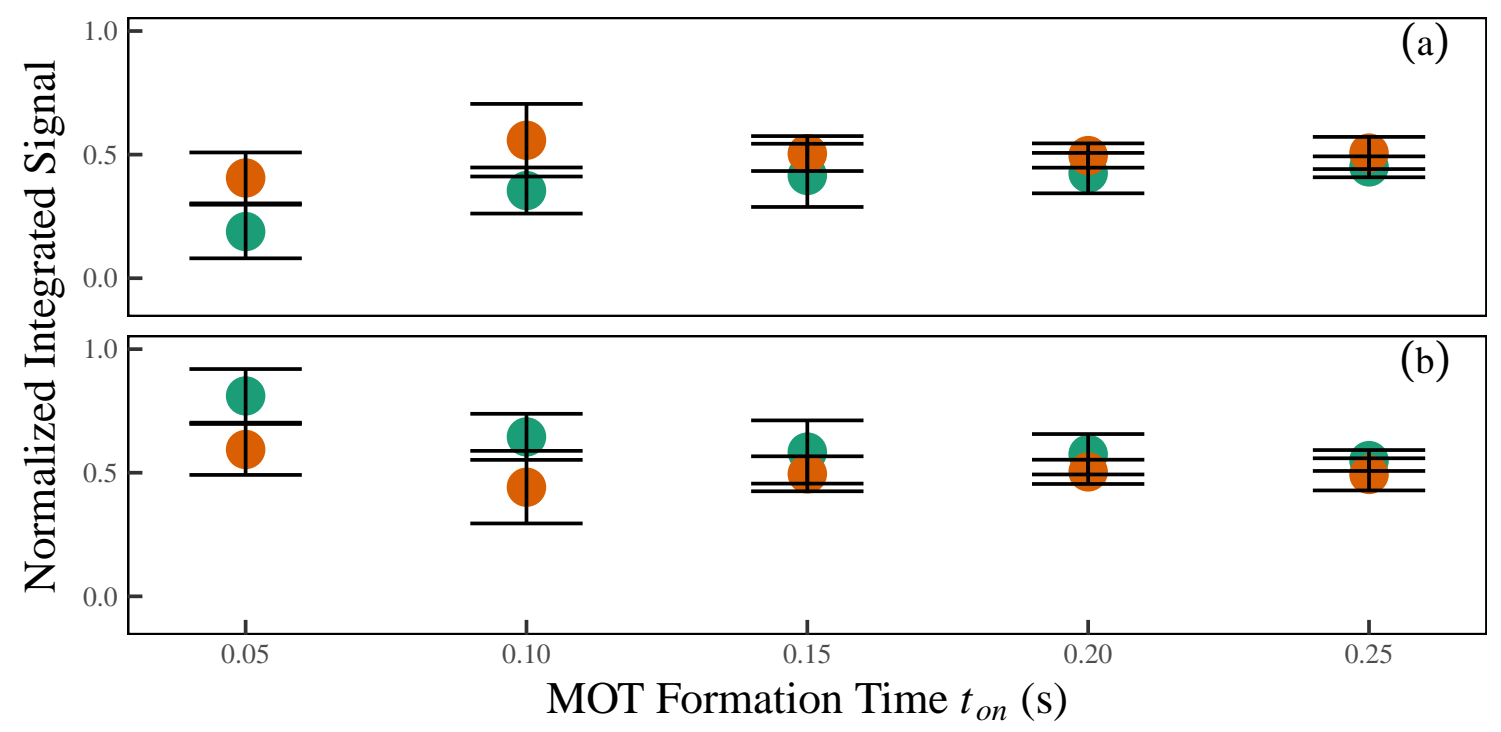

Figure 5.13: (a) Normalized Rydberg population and (b) normalized ion population for a control laser detuning $\Delta=30 \mathrm{MHz}$. Measurements were made at electric fields of $F=$ $12.6 \mathrm{~V} / \mathrm{cm}$ (green points) and $18.6 \mathrm{~V} / \mathrm{cm}$ (orange points). Both Rydberg and ion populations were normalized by dividing by the total ion yield (sum of the Rydberg and ion populations). 


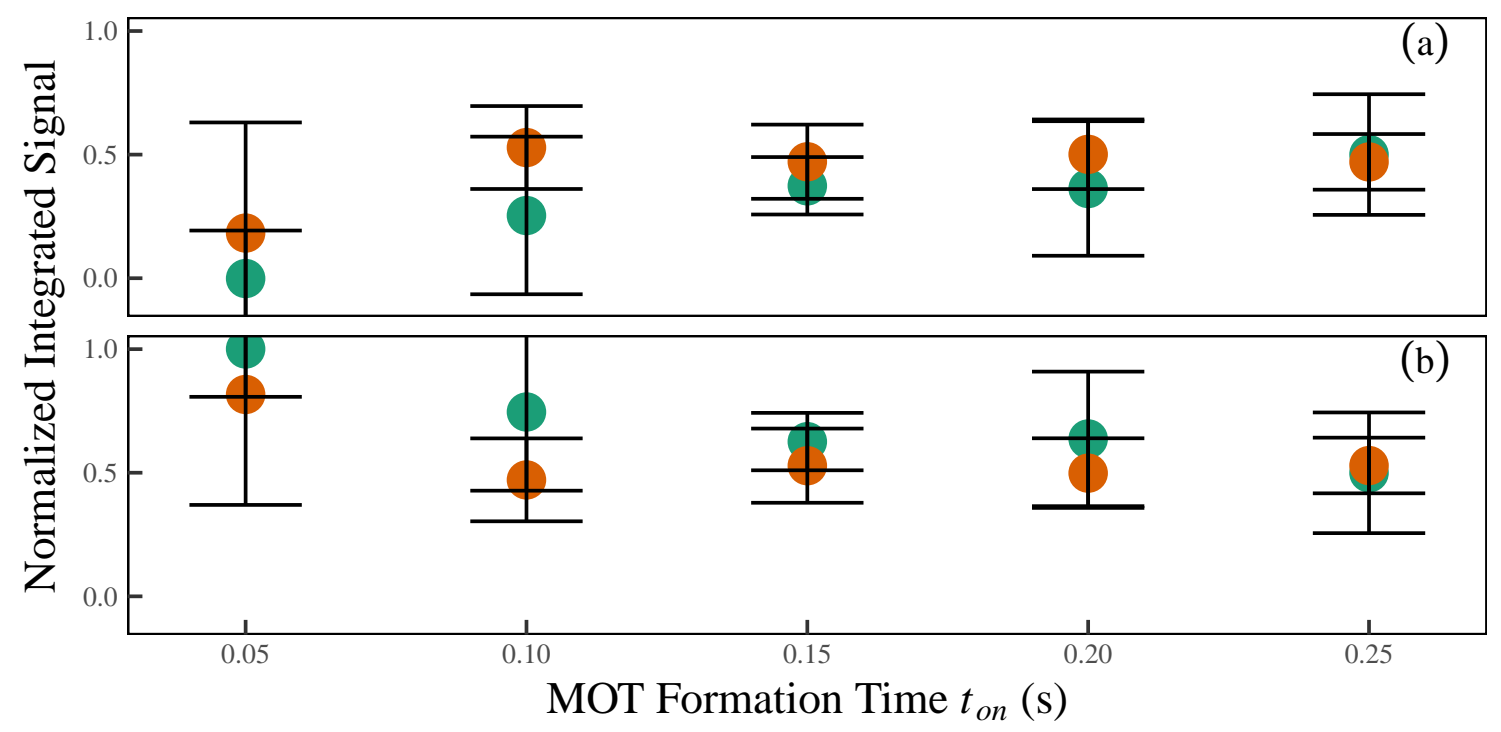

Figure 5.14: (a) Normalized Rydberg population and (b) normalized ion population for a control laser detuning $\Delta=40 \mathrm{MHz}$. Measurements were made at electric fields of $F=$ $12.6 \mathrm{~V} / \mathrm{cm}$ (green points) and $18.6 \mathrm{~V} / \mathrm{cm}$ (orange points). Both Rydberg and ion populations were normalized by dividing by the total ion yield (sum of the Rydberg and ion populations). 
where $C=\frac{2}{\sqrt{3}} \mu_{A} \mu_{B}$ and $v$ is the relative speed of the atoms [22]. Figure 5.15 shows $P_{\text {adiabatic }}$ as a function of the Rabi frequency of the control laser for a few typical detunings and a typical relative velocity of atoms in the MOT of $v=0.22 \mathrm{~m} / \mathrm{s}(\sim 70 \mu \mathrm{K})$. Obtaining $\sim 100 \%$ adiabatic transfer would require a Rabi frequency of $\Omega \sim 15 \mathrm{MHz}$ for detunings of 30 or $40 \mathrm{MHz}$. These higher Rabi frequencies are needed at large detuning due to the increasing steepness of the Rydberg-Rydberg curve with decreasing $R$. Due to the limitations of the laser equipment and focusing used in these experiments, we achieved a maximum Rabi frequency of $\Omega \sim 4 \mathrm{MHz}$. As can be seen in Figure 5.15, this Rabi frequency is clearly not in the purely adiabatic crossing regime at larger detunings.

\subsection{Model Improvements}

Our model for controlling the minimum separation of atoms is a simple one that neglects four important factors: the $5 s_{1 / 2}$ ground state of ${ }^{85} \mathrm{Rb}$, spontaneous decay from the $5 p_{3 / 2}$ trap state to the $5 s_{1 / 2}$ ground state, the natural energy width of the $5 p_{3 / 2}$ trap state, and the orientation of atom pairs relative to the laser polarization. As such, we must consider these factors if we hope to observe minimum atom separation control.

First, let us include the $5 s_{1 / 2}$ state in our model. Adding the $5 s_{1 / 2}$ state gives us three new pair states, $5 s_{1 / 2} 5 s_{1 / 2}, 5 s_{1 / 2} 5 p_{3 / 2}$, and $5 p_{3 / 2} 5 s_{1 / 2}$, that we must include in our laser-dressed atom pair calculation. The $5 p_{3 / 2}$ state is detuned from the $5 s_{1 / 2} \rightarrow 5 p_{3 / 2}$ transition by $\Delta_{1}$ and is laser-coupled to the $5 s_{1 / 2}$ state with a single-atom Rabi frequency $\Omega_{1}$. Figure 5.16(a) shows the energy levels of this new system, as a function of atom pair separation, for a trap laser with red detuning $\Delta_{1}=20 \mathrm{MHz}$ and single-atom Rabi frequency $\Omega_{1}=16 \mathrm{MHz}$ for 


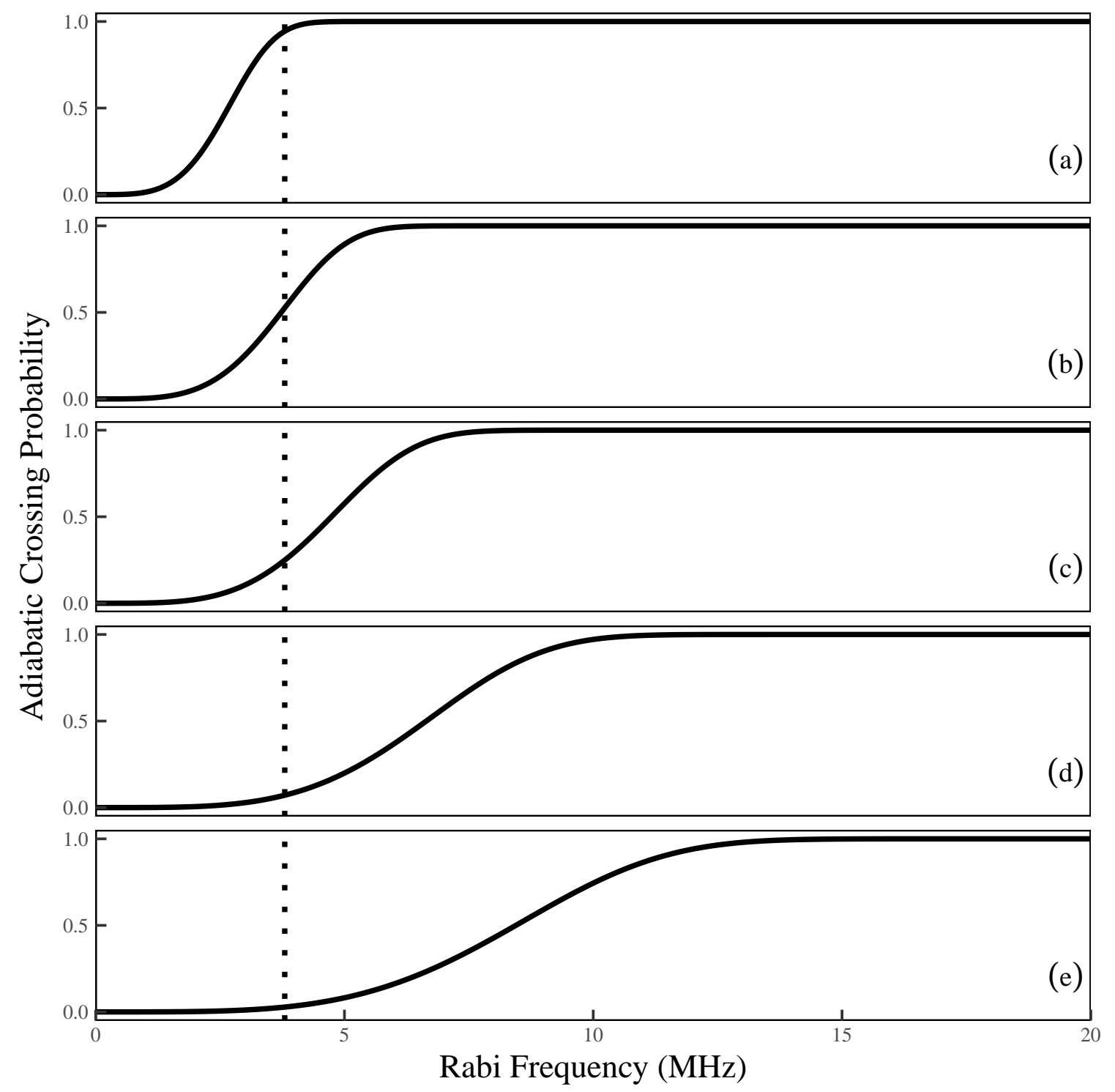

Figure 5.15: Adiabatic crossing probability as a function of Rabi Frequency $\Omega$ for control laser detunings $\Delta$ of (a) $10 \mathrm{MHz}$, (b) $15 \mathrm{MHz}$, (c) $20 \mathrm{MHz}$, (d) $30 \mathrm{MHz}$, and (e) $40 \mathrm{MHz}$. The adiabatic crossing probability is given in Equation 5.1. The dotted vertical line is a reference for the control laser Rabi frequency used in the experiments, approximately 4 $\mathrm{MHz}$. 
the laser-atom coupling between the $5 s_{1 / 2}$ and $5 p_{3 / 2}$ states. For this calculation, we assume a fixed blue detuning $\Delta_{2}=30 \mathrm{MHz}$ from the Stark-shifted $5 p_{3 / 2} \rightarrow 32 p_{3 / 2}$ transition and a single-atom Rabi frequency $\Omega_{2}=22 \mathrm{MHz}$ for the laser-atom coupling between the $5 p_{3 / 2}$ and $32 p_{3 / 2}$ states.

Figure 5.16(b) - (f) shows that atoms primarily in the $5 s 5 p+5 p 5 s, 5 p 5 p$, and $5 p 32 p+$ $32 p 5 p$ states should adiabatically transition to the repelling $32 p 32 p+32 s 33 s$ Rydberg state. For atom temperatures of $\sim 70 \mu \mathrm{K}$, the atoms will have a kinetic energy equivalent to $\sim 2$ MHz. At these temperatures, atoms in states with some $5 p$ character should be stopped by the DD interaction due to the steep slope. Atoms in the bottom energy level with mostly $5 s 5 s$ character are less likely to make the adiabatic transition due to the reduced coupling to the repelling $32 p 32 p+32 s 33 s$ Rydberg state. If the adiabatic transition occurs, then the energy shift of the repelling Rydberg state should be enough to stop those atoms as well. Overall, a less well-defined minimum atom separation is expected as the atoms on different energy levels make adiabatic transitions at different separations. While the separation may not be well defined and not all of the atoms may be stopped, we still might expect enough atoms to be affected to alter the atom correlation function, reducing the number of atom pairs with very small separations.

Second, let us consider spontaneous decay from the $5 p_{3 / 2}$ trap state to the $5 s_{1 / 2}$ ground state. Atoms traversing the avoided crossings in states that contain $5 p_{3 / 2}$ character could decay during the crossing. If those atoms are cycled back into the $5 p_{3 / 2}$ state, the atoms would have some probability of being in either state that makes up the avoided crossing. Atoms on the lower state of the avoided crossing would have nothing preventing them from approaching closer than the minimum separation. Atom pairs traverse the highest avoided 


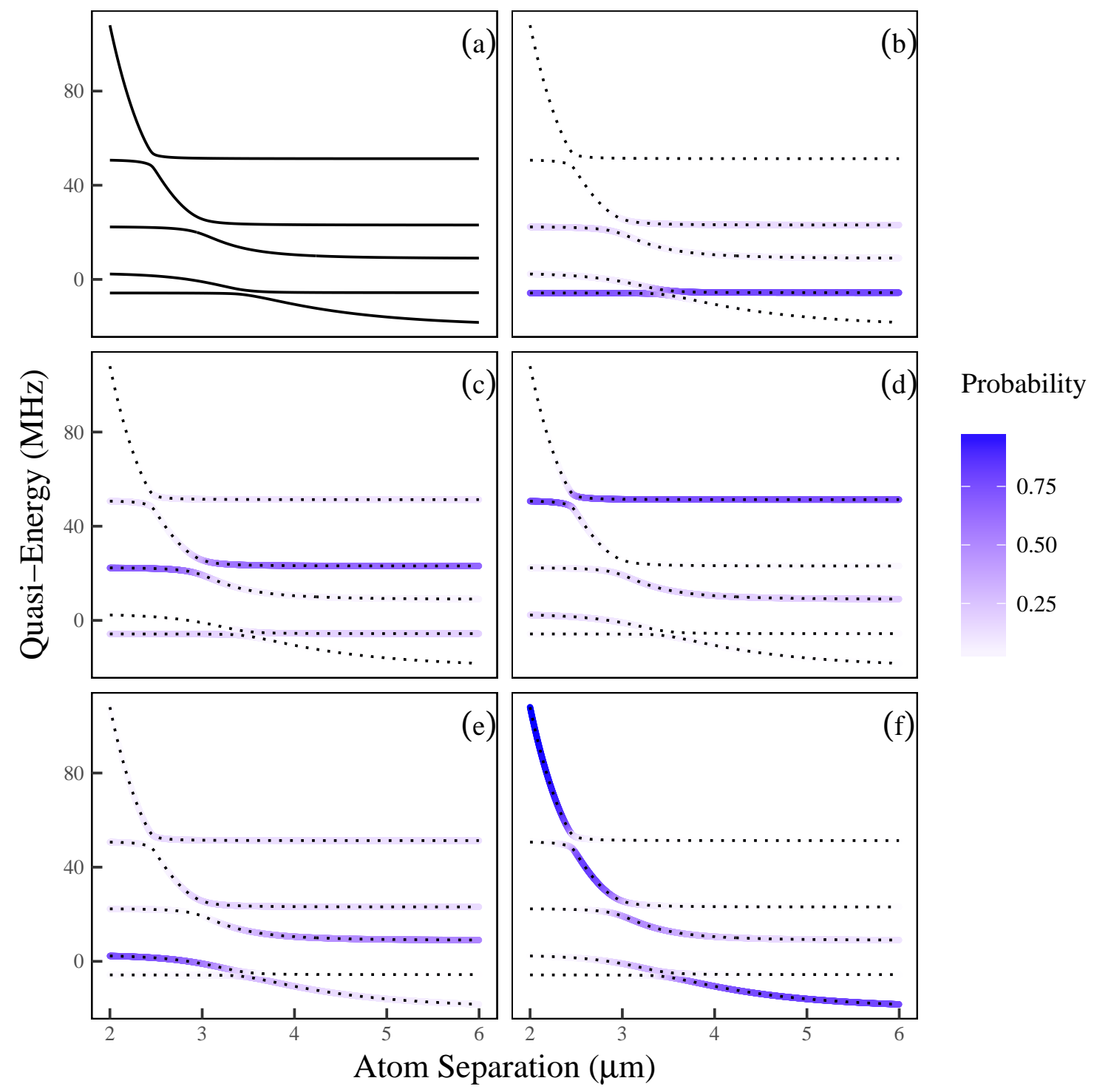

Figure 5.16: Energy of the laser dressed, DD resonant, atom pair for a model including the $5 s$ ground state. The calculation was done using a trap laser detuning $\Delta_{1}=20 \mathrm{MHz}$ and single-atom Rabi frequency $\Omega_{1}=16 \mathrm{MHz}$ and a control laser detuning $\Delta_{2}=30 \mathrm{MHz}$ and single-atom Rabi frequency $\Omega_{2}=22 \mathrm{MHz}$. The energy levels are shown in the solid lines in (a). (b) - (f) shows the energy levels in the dashed lines with the color giving the probability for the atom pairs to be in the state (b) $5 s 5 s$, (c) $5 s 5 p+5 p 5 s$, (d) $5 p 5 p$, (e) $5 p 32 p+32 p 5 p$, (f) $32 p 32 p+32 s 33 s$. The repelling Rydberg-Rydberg state $32 p 32 p-32 s 33 s$, along with the non-interacting, "dark", states $5 s 5 p-5 p 5 s$ and $5 p 32 p-32 p 5 p$ are not shown. 
crossing in $\sim 1 \mu \mathrm{s}$, giving the atoms many opportunities to spontaneously decay with a $5 p_{3 / 2}$ lifetime of approximately $26 \mathrm{~ns}$. Even with these losses, there may be enough atoms repelling at the minimum separation to create an observable effect. Accurately treating the effect of spontaneous emission would require a quantum Monte Carlo simulation or density matrix calculation that is beyond the work presented in this dissertation.

Third, let us consider the natural energy width of the $5 p_{3 / 2}$ trap state and examine the uppermost avoided crossing from Figure 5.16. Due to the natural energy width of the $5 p_{3 / 2}$ state, atoms with $5 p_{3 / 2}$ character can pass through the avoided crossing without making the transition to the repelling Rydberg curve for small laser-atom couplings. By making the avoided crossing gap larger than the natural energy width of the $5 p_{3 / 2}$ state, we can ensure that there is no overlap between the states on either side of the avoided crossing. With an avoided crossing gap of $\sqrt{2} \Omega^{2} /(4 \Delta)$ [22], we would need a Rabi frequency of $\sim 21 \mathrm{MHz}$ at a typical detuning of $30 \mathrm{MHz}$. Figure 5.17 shows a rough calculation of the laser-dressed energies as shown in Figure 5.16, but with a single-atom Rabi frequency $\Omega$ of $22 \mathrm{MHz}$ and including a natural width for the $5 p_{3 / 2}$ trap state. This calculation was done by diagonalizing a time-independent Hamiltonian as for Figure 5.16, but adding the complex term $i \Gamma / 2$ to all of the diagonal terms with a single atom in the $5 p_{3 / 2}$ state and adding the complex term $i \Gamma$ to the state with both atoms in the $5 p_{3 / 2}$ state, where $\Gamma=6.0666 \mathrm{MHz}$ is the natural width of $5 p_{3 / 2}$. In Figure 5.17, the solid black lines are the average energies of each state given by the real component of the diagonalized energies. The gray bars are the width of each state given by $\pm 1 / 2$ of the imaginary component of the diagonalized energies. A laser-atom coupling of $\Omega=22 \mathrm{MHz}$ clearly creates a large enough avoided crossing gap to fully separate the states. 


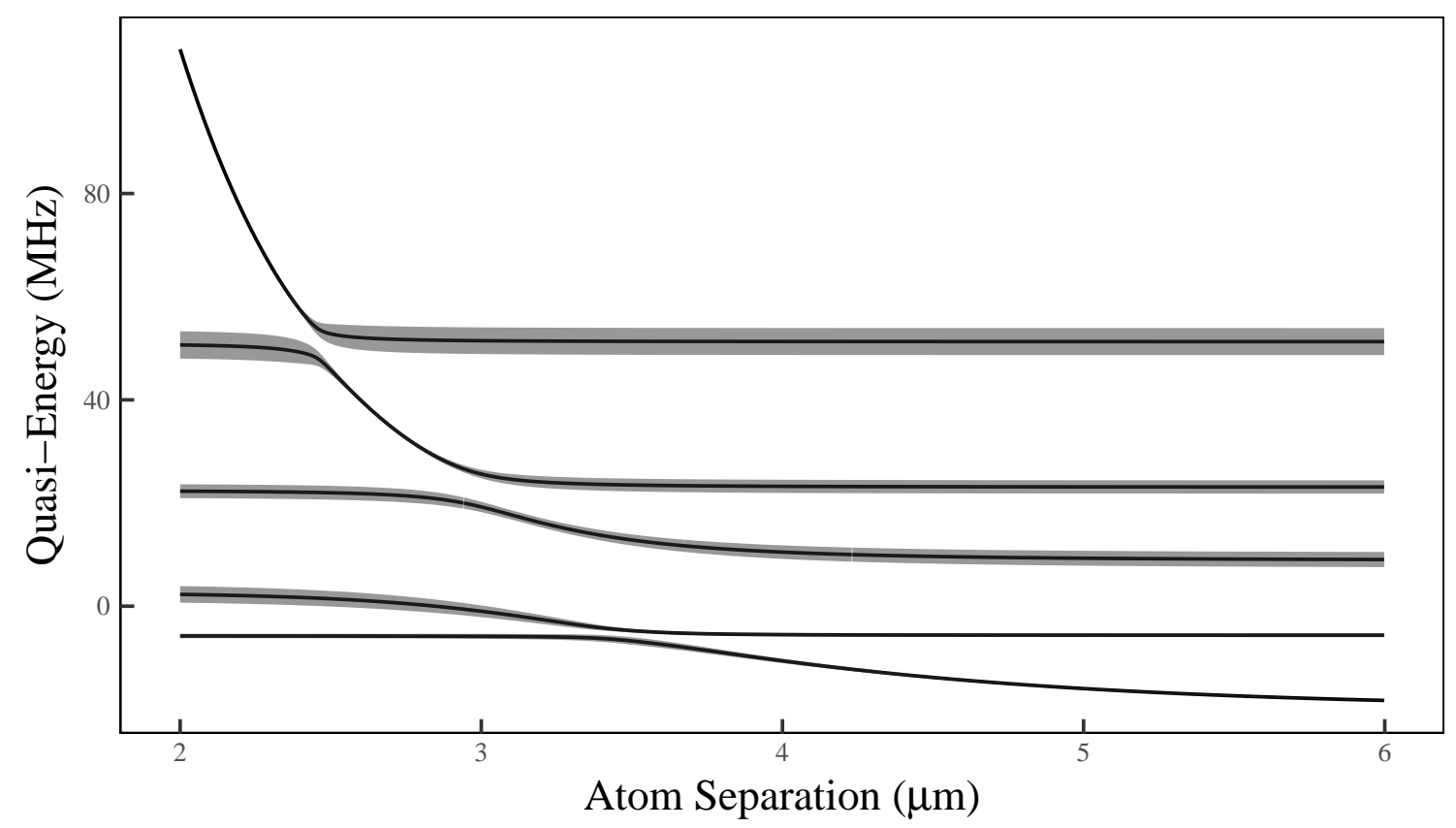

Figure 5.17: Rough model showing the laser dressed, DD resonant, atom pair energies and including the $5 s$ ground state and the natural energy width of the $5 p_{3 / 2}$ state. The calculation was done using a trap laser detuning $\Delta_{1}=20 \mathrm{MHz}$ and single-atom Rabi frequency $\Omega_{1}=16 \mathrm{MHz}$ and a control laser detuning $\Delta_{2}=30 \mathrm{MHz}$ and single-atom Rabi frequency $\Omega_{2}=22 \mathrm{MHz}$. The bold black lines are the average energies for each state. The gray bars around each line are the energy widths of each state assuming the natural line width for the $5 p_{3 / 2}$ state of $6.0666 \mathrm{MHz}$ [7].

Fourth, we must consider the effect of atom pair orientation, relative to the control laser polarization, on the laser-atom coupling. As discussed by Wall et al., there are possible atom pair orientations that could result in a negligible coupling between the control laser and the atoms [22]. This means that atom pairs with those orientations will be unable to transition to the repelling Rydberg state and can move closer than the minimum separation. Wall et al. suggested a few methods for limiting these non-interacting configurations, including using a restricted geometry, so that atoms only approach one another from angles away from the 
non-interacting configurations, and using a different control laser polarization [22]. While neither of these suggestions were implemented in the experiments described in Sections 5.2 and 5.3, we had hoped that a sufficient number of atoms would be oriented such that we could observe some signature of minimum atom separation control.

\subsection{Future Steps}

To move forward with these methods for minimum atom separation control, some improvements must be made to increase the likelihood of observing a signature of minimum atom separation control. As discussed in Section 5.3.2, the laser-atom coupling was likely not large enough to allow for purely adiabatic transitions to the repelling Rydberg state. To reach the adiabatic atom separation control regime, we must address two primary factors: the control laser detuning and power. Figure 5.15(d) shows that at a detuning of $\Delta=$ $30 \mathrm{MHz}$, we would need a Rabi frequency of $\Omega \sim 15 \mathrm{MHz}$ to enter the purely adiabatic regime. The adiabatic passage requirement and the requirement that the Rabi frequency be at least $\sim 21 \mathrm{MHz}$ to create a large enough avoided crossing gap means that we will need a laser-atom coupling of at least $\sim 21 \mathrm{MHz}$. At our current maximum power of $P=50 \mathrm{~mW}$, we get a laser-atom coupling with a Rabi frequency of $\Omega \sim 4 \mathrm{MHz}$ for a laser spot size of $0.1 \mathrm{~mm}$ using a $f=350 \mathrm{~mm}$ converging lens. To reach the adiabatic regime would require increasing the intensity of the laser at the focus by a factor of $\sim 28$ since the Rabi frequency scales as the square root of the laser intensity. Doing so by increasing the power of the laser alone would require a laser power of $P \approx 1.4 \mathrm{~W}$, which is well outside the range of our current equipment. Another choice would be to decrease the spot size of the laser at the 
focus. This could be done by increasing the size of the laser beam when it hits the focusing lens or by decreasing the focal length of the lens. By increasing the spot size of the laser beam on the $350 \mathrm{~mm}$ focusing lens by a factor of 5 , the spot size at the focus will decrease by a factor of 5 . This gives a factor of 25 increase in the laser intensity at the focus, which should hopefully allow us to reach the purely adiabatic regime.

Another possibility is to lower the detuning of the control laser. Figure 5.15(a) shows that for a detuning $\Delta=10 \mathrm{MHz}$ a Rabi Frequency of $\Omega=7 \mathrm{MHz}$ should be sufficient to ensure that the interaction is purely adiabatic. Additionally, a lower detuning will give us a much larger minimum separation. With the current excitation scheme, lower detunings are not feasible due to direct excitation of the $32 p_{3 / 2}$ Rydberg state from the broad $5 p_{3 / 2}$ trap state. A lower detuning could be accessed by the introduction of a $\sim 780 \mathrm{~nm}$ laser far detuned from the $5 p_{3 / 2} F=4$ resonance. Using a two-photon transition to access the $32 p_{3 / 2}$ state, a much lower detuning could be achieved. In addition to the lower net detuning, a two-photon transition would allow us to work with a much smaller avoided crossing gap, as the large detuning from the $5 p_{3 / 2}$ state will reduce the impact of it's broad energy width. However, this will require an even larger laser intensity due to the smaller Rydberg coupling via the two-photon excitation. Additionally, such a large detuning could put the lasers close to other resonances involving different $5 p_{3 / 2}$ hyperfine components as detailed in Section 5.2.2.

While increasing the control laser intensity should help ensure that the atoms are able to make adiabatic transitions to the repelling Rydberg curve, a large laser intensity alone will not solve all of the complications introduced in the expanded model discussed in Section 5.4. However, it should help to increase our chances of observing any signature of minimum 
atom separation control.

In addition to the major changes in laser intensity discussed above, the following improvements are recommended to ensure higher quality measurements and are meant as a guide to future students within our lab. Two factors contributing to measurement noise are the control laser frequency stability and the re-pump laser switch stability. Instability in the control laser frequency prevents longer and repeated measurements that could be used to reduce the overall noise in the measurements. This instability appears to primarily come from transient fluctuations in the piezoelectric that controls the cavity length of the Fabry-Pérot interferometer (as described in Section 2.3.5). The long settling time of the piezoelectric causes any sudden changes in the voltage applied to the piezoelectric to cause a drift in the laser frequency. The control laser frequency instability can be reduced with the introduction of a more robust frequency stabilization system.

Intermittent instability in the re-pump laser switch prevents the MOT from fully dissipating, causing the control laser to be introduced with atoms already present. This appears to be due to leakage of the un-switched re-pump laser beam in the AOM switch that arises from small changes in the re-pump laser pointing. This can be improved by expanding and collimating the re-pump laser beam prior to the AOM. For the measurements shown in Figures 5.12, 5.13, and 5.14, the re-pump beam was too small and slightly expanding, causing some parts of the beam to not meet the Bragg condition and thus not be switched by the AOM. By expanding and better collimating the re-pump laser beam, it should be easier to separate the beams and eliminate any leakage from the AOM. Another alternative is to introduce an AOM into the trap laser beam, ensuring the complete dissipation of the MOT by removing the trap laser. The downside to introducing an AOM to the trap laser beam is 
a loss of trap laser power that may prevent the formation of the MOT.

\subsection{Conclusion}

Though limitations in control laser power may have prevented us from achieving purely adiabatic atom separation control interactions, we have explored two methods for atom separation control. The chirped-frequency method involved illuminating a fully-loaded MOT with a control laser at larger detuning and chirping it towards the atomic resonance. We determined that the chirped-frequency method is unlikely to achieve measurable atom separation control with our current apparatus due to the large initial detuning and chirping range required to separate closely spaced atoms. The fixed-frequency method involved forming the MOT while it is illuminated with a control laser beam of fixed frequency, preventing the atoms from approaching closer than the minimum separation. We determined that the fixed-frequency method is more likely to achieve atom separation control once the control laser power and detuning limitations have been solved. Additional measurement of the atomic spectrum around the $32 p_{3 / 2}$ Rydberg state and the laser pulse length dependence of the Rydberg and ion populations have given us further information on how to design future experiments to improve the likelihood of achieving atom separation control. 


\section{Chapter 6}

\section{Summary and Conclusion}

Over the last few decades, the study of dipole-dipole interactions in cold Rydberg gases has been rapidly growing. Dipole-dipole interactions offer opportunities to study few- to many-body physics and quantum information. The study of few-body physics is the primary focus of the experiments discussed here.

Chapter 3 described measurements of atomic decay rates in a cold Rydberg gas. We concluded that the lack of superradiant or collective decay can be attributed to strong dipole-dipole interactions causing variations in energy levels across the MOT. Additional measurements showed that the electric field inhomogeneity was small enough to not effect the decay for $s$-state atoms, but were comparable with the dipole-dipole force for $p$-state atoms. Our measurements were consistent with spontaneous emission from, and blackbody redistribution within, isolated atoms, showing no sign of superradiant emission.

In Chapter 4, we described measurements of the line shape of the dipole-dipole mediated resonant energy transfer reaction $32 p_{3 / 2} 32 p_{3 / 2} \leftrightarrow 32 s 33 s$. We observed cusp-like shapes at 
high Rydberg atom densities, reflecting the random nearest-neighbor distribution of atoms in the MOT. These are well reproduced by a two-body model. Resonance widths agree with the model for interaction times $\tau<1 \mu$ s with broadening by a factor of 2 for interaction times of $\tau=15 \mu \mathrm{s}$. We attribute this broadening to atomic motion. We additionally conclude that beyond nearest-neighbor processes do not contribute to line shape broadening at the scale previously thought $[10,11]$.

In Chapter 5, we explore methods to control the minimum separation of atoms using dipole-dipole forces. This is done by the introduction of a strong, blue-detuned, control laser to the MOT, creating a set of Rydberg dressed states. We first described a method using a chirped-frequency control laser to push atoms at small separations to larger ones over the course of the frequency chirp. This method was deemed ineffective with the current apparatus due to the large detuning of $500 \mathrm{MHz} \lesssim \Delta \lesssim 5 \mathrm{GHz}$ required to influence the most closely spaced atoms. The second method described uses a fixed-frequency control laser that prevents atoms from going beyond the minimum separation while the MOT is filled. This method appears to be more promising, though limitations in control laser intensity may be responsible for preventing us from observing atom separation control.

In the future, the decay measurements of Chapter 3 might be expanded by considering states of even lower $n$. The superradiant decay and dipole-dipole dephasing rates are essentially identical up to a geometric factor of $L / \lambda$ in the superradiance rate, where $L$ is the excitation length and is equal to the MOT diameter. As $n$ decreases, $\lambda$ associated with the lowest energy transition also decreases, allowing for superradiant decay to out pace dipole-dipole dephasing. By stepping down in $n$, we expect that we should enter a regime where we observe an enhanced decay rate. 
The fixed-frequency atom separation control method described in Chapter 5 can be improved two ways. First, the intensity of the laser will have to be increased by a factor of $\sim 28$ to reach a Rabi frequency allowing for purely adiabatic transitions. Second, a $\sim 780 \mathrm{~nm}$ laser with large detuning from the $5 p_{3 / 2}$ state can be introduced to allow for two-photon excitation to the $32 p_{3 / 2}$ state without any direct excitation of the broad $5 p_{3 / 2}$ state. This method will also require a large intensity in both lasers, but it has the added benefit of allowing for smaller detuning from Rydberg resonance. Lower detuning allows for a larger minimum separation and a lower Rabi frequency required to reach the purely adiabatic transition regime. With a functioning minimum atom separation control method, separation-dependent phenomena, such as the line shapes of DD resonances as described in Chapter 4 and wavepacket coherence transfer via DD interactions [45], might be studied with a more uniform ensemble of atoms. 


\section{Bibliography}

[1] New Focus. User's Guide: 7000/6000 Series Tunable Diode Laser. San Jose, CA, USA, 2007.

[2] Continuum. Operation and Maintenance Manual for Surelite Lasers. Santa Clara, CA, USA, 1997.

[3] H. J. Metcalf and P van der Stratten. Laser Cooling and Trapping. Springer-Verlag New York, Inc., New York, NY, USA, 1999.

[4] E. L. Raab, M. Prentiss, Alex Cable, Steven Chu, and D. E. Pritchard. Trapping of Neutral Sodium Atoms with Radiation Pressure. Phys. Rev. Lett., 59, 2631, 1987.

[5] M. R. Kutteruf. PhD thesis, University of Virginia, 2010.

[6] Tao Zhou, B. G. Richards, and R. R. Jones. Absence of Collective Decay in a Cold Rydberg Gas. 93, 033407, 2016.

[7] Daniel A. Steck. Rubidium 85 d line data. Available online at http://steck.us/alkalidata (revision 2.1.5, 19 September 2012). 
[8] T. F. Gallagher. Rydberg Atoms. Cambridge University Press, Cambridge, England, 1994.

[9] B. Sun and F. Robicheaux. Spectral linewidth broadening from pair fluctuations in a frozen Rydberg gas. Phys. Rev. A, 78, 040701(R), 2008.

[10] W. R. Anderson, J. R. Veale, and T. F. Gallagher. Resonant dipole-dipole energy transfer in a nearly frozen Rydberg gas. Phys. Rev. Lett., 80, 249, 1998.

[11] I. Mourachko, D. Comparat, F. de Tomasi, A. Fioretti, P. Nosbaum, V. M. Akulin, and P. Pillet. Many-body effects in a frozen Rydberg gas. Phys. Rev. Lett., 80, 253, 1998.

[12] M. D. Lukin, M. Fleischhauer, R. Cote, L. M. Duan, D. Jaksch, J. I. Cirac, and P. Zoller. Dipole Blockade and Quantum Information Processing in Mesoscopic Atomic Ensembles. Phys. Rev. Lett., 87, 037901, 2001.

[13] W. R. Anderson, M. P. Robinson, J. D. D. Martin, and T. F. Gallagher. Dephasing of resonant energy transfer in a cold Rydberg gas. Phys. Rev. A, 65, 063404, 2002.

[14] D. Tong, S. M. Farooqi, J. Stanojevic, S. Krishnan, Y. P. Zhang, R. Côté, E. E. Eyler, and P. L. Gould. Local blockade of Rydberg excitation in an ultracold gas. Phys. Rev. Lett., 93, 063001, 2004.

[15] I. Mourachko, Wenhui Li, and T. F. Gallagher. Controlled many-body interactions in a frozen Rydberg gas. Phys. Rev. A, 70, 031401(R), 2004. 
[16] Thomas J. Carroll, Katharine Claringbould, Anne Goodsell, M. J. Lim, and Michael W. Noel. Angular dependence of the dipole-dipole interaction in a nearly one-dimensional sample of Rydberg atoms. Phys. Rev. Lett., 93, 153001, 2004.

[17] Kilian Singer, Markus Reetz-Lamour, Thomas Amthor, Luis Gustavo Marcassa, and Matthias Weidemüller. Suppression of excitation and spectral broadening induced by interactions in a cold gas of Rydberg atoms. Phys. Rev. Lett., 93, 163001, 2004.

[18] F. Robicheaux and J. V. Hernández. Many-body wave function in a dipole blockade configuration. Phys. Rev. A, 72, 063403, 2005.

[19] T. Cubel Liebisch, A Reinhard, P. R. Berman, and G. Raithel. Atom counting statistics in ensembles of interacting Rydberg atoms. Phys. Rev. Lett., 95, 253002, 2005.

[20] S. Westermann, T. Amthor, A. L. de Oliveira, J. Deiglmayr, M. Reetz-Lamour, and M. Weidemüller. Dynamics of resonant energy transfer in a cold Rydberg gas. Eur. Phys. J. D, 40, 37, 2006.

[21] Thibault Vogt, Matthieu Viteau, Jianming Zhao, Amodsen Chotia, Daniel Comparat, and Pierre Pillet. Dipole blockade at Förster resonances in high resolution laser excitation of Rydberg states of cesium atoms. Phys. Rev. Lett., 97, 083003, 2006.

[22] M. L. Wall, F. Robicheaux, and R. R. Jones. Controlling atom motion through the dipole-dipole force. J. Phys. B At. Mol. Opt. Phys., 40, 3693, 2007.

[23] C. S. E. Van Ditzhuijzen, A. F. Koenderink, J. V. Hernández, F. Robicheaux, L. D. Noordam, and H. B Van Linden Van Den Heuvell. Spatially resolved observation 
of dipole-dipole interaction between Rydberg atoms. Phys. Rev. Lett., 100, 243201, 2008.

[24] K. C. Younge, A. Reinhard, T. Pohl, P. R. Berman, and G. Raithel. Mesoscopic Rydberg ensembles: Beyond the pairwise-interaction approximation. Phys. Rev. A, 79, 043420, 2009.

[25] Alpha Gaëtan, Yevhen Miroshnychenko, Tatjana Wilk, Amodsen Chotia, Matthieu Viteau, Daneil Comparat, Pierre Pillet, Antoine Browaeys, and Philippe Grangier. Observation of collective excitation of two individual atoms in the Rydberg blockade regime. Nat. Phys., 5, 115, 2009.

[26] E. Urban, T. A. Johnson, T. Henage, L. Isenhower, D. D. Yavuz, T. G. Walker, and M. Saffman. Observation of Rydberg blockade between two atoms. Nat. Phys., 5, $110,2009$.

[27] Robert Löw, Hendrik Weimer, Ulrich Krohn, Rolf Heidemann, Vera Bendkowsky, Björn Butscher, Hans Peter Büchler, and Tilman Pfau. Universal scaling in a strongly interacting Rydberg gas. Phys. Rev. A, 80, 033422, 2009.

[28] T. Pohl and P. R. Berman. Breaking the dipole blockade: Nearly resonant dipole interactions in few-atom systems. Phys. Rev. Lett., 102, 013004, 2009.

[29] I. I. Ryabtsev, D. B. Tretyakov, I. I. Beterov, and V. M. Entin. Observation of the stark-tuned Förster resonance between two Rydberg atoms. Phys. Rev. Lett., 104, 073003, 2010. 
[30] T. Pohl, E. Demler, and M. D. Lukin. Dynamical crystallization in the dipole blockade of ultracold atoms. Phys. Rev. Lett., 104, 043002, 2010.

[31] S. Wüster, C. Ates, A. Eisfeld, and J. M. Rost. Newton's cradle and entanglement transport in a flexible Rydberg chain. Phys. Rev. Lett., 105, 053004, 2010.

[32] J. D. Pritchard, D. Maxwell, A. Gauguet, K. J. Weatherill, M. P. A. Jones, and C. S. Adams. Cooperative atom-light interaction in a blockaded Rydberg ensemble. Phys. Rev. Lett., 105, 193603, 2010.

[33] A. Schwarzkopf, R. E. Sapiro, and G. Raithel. Imaging spatial correlations of Rydberg excitations in cold atom clouds. Phys. Rev. Lett., 107, 103001, 2011.

[34] F. Bariani, Y. O. Dudin, T. A. B. Kennedy, and A. Kuzmich. Dephasing of multiparticle Rydberg excitations for fast entanglement generation. Phys. Rev. Lett., 108, 030501, 2012.

[35] Matthieu Viteau, Paul Huillery, Mark G. Bason, Nicola Malossi, Donatella Ciampini, Oliver Morsch, Ennio Arimondo, Daniel Comparat, and Pierre Pillet. Cooperative excitation and many-body interactions in a cold Rydberg gas. Phys. Rev. Lett., 109, 053002, 2012.

[36] M. R. Kutteruf and R. R. Jones. Probing electronic coherence in a gas of dipole-dipole coupled rydberg atoms. Phys. Rev. Lett., 108, 013001, 2012.

[37] H. Schempp, G. Günter, M. Robert-De-Saint-Vincent, C. S. Hofmann, D. Breyel, A. Komnik, D. W. Schönleber, M. Gärttner, J. Evers, S. Whitlock, and M. Wei- 
demüller. Full counting statistics of laser excited Rydberg aggregates in a onedimensional geometry. Phys. Rev. Lett., 112, 013002, 2014.

[38] Daniel Tiarks, Simon Baur, Katharina Schneider, Stephan Dürr, and Gerhard Rempe. Single-photon transistor using a Förster resonance. Phys. Rev. Lett., 113, 053602, 2014.

[39] Y. O. Dudin and A. Kuzmich. Excitations of a Cold Atomic Gas. Science, 336, 887, 2012.

[40] M. Saffman, T. G. Walker, and K. Mølmer. Quantum information with Rydberg atoms. Rev. Mod. Phys., 82, 2313, 2010.

[41] L. Isenhower, E. Urban, X. L. Zhang, A. T. Gill, T. Henage, T. A. Johnson, T. G. Walker, and M. Saffman. Demonstration of a neutral atom controlled-NOT quantum gate. Phys. Rev. Lett., 104, 010503, 2010.

[42] D. Paredes-Barato and C. S. Adams. All-optical quantum information processing using Rydberg gates. Phys. Rev. Lett., 112, 040501, 2014.

[43] M. Ebert, M. Kwon, T. G. Walker, and M. Saffman. Coherence and Rydberg Blockade of Atomic Ensemble Qubits. Phys. Rev. Lett., 115, 093601, 2015.

[44] T. Wang, S. F. Yelin, R. Côté, E. E. Eyler, S. F. Farooqi, P. L. Gould, M. Koštrun, D. Tong, and D. Vrinceanu. Superradiance in ultracold Rydberg gases. Phys. Rev. A, 75, 033802, 2007.

[45] T. Zhou. PhD thesis, University of Virginia, 2016. 
[46] D. R. Hartree. The Wave Mechanics of an Atom with a Non-Coulomb Central Field. Part I. Theory and Methods. Math. Proc. Cambridge Philos. Soc., 24, 89, 1928.

[47] H. A. Bethe and E. E. Salpeter. Quantum Mechanics of One- and Two-Electron Atoms. Dover Publications, Inc., Mineola, New York, USA, 2008.

[48] F. Weinhold and C. R. Landis. Discovering Chemistry With Natural Bond Orbitals. John Wiley \& Sons, Inc., Hoboken, New Jersey, USA, 2012.

[49] K. A. Safinya, J. F. Delpech, F. Gounand, W. Sandner, and T. F. Gallagher. Resonant Rydberg-atom-Rydberg-atom collisions. Phys. Rev. Lett., 47, 405, 1981.

[50] Michael G. Littman and Harold J. Metcalf. Spectrally narrow pulsed dye laser without beam expander. Appl. Opt., 17, 2224, 1978.

[51] K. C. Harvey and C. J. Myatt. External-cavity diode laser using a grazing-incidence diffraction grating. Opt. Lett., 16, 910, 1991.

[52] K. Liu and M. G. Littman. Novel geometry for single-mode scanning of tunable lasers. Opt. Lett., 6, 117, 1981.

[53] Toptica Photonics AG. DL pro: Grating Stabilized Diode Laser Head Manual. Munich, Germany, 2008.

[54] Toptica Photonics AG. TA/DL-SHG pro: Frequency Doubled High Power Laser System Manual. Munich, Germany, 2009. 
[55] R. W. P. Drever, J. L. Hall, F. V. Kowalski, J. Hough, G. M. Ford, A. J. Munley, and H. Ward. Laser phase and frequency stabilization using an optical resonator. Appl. Phys. B, 31, 97, 1983.

[56] Eric D. Black. An introduction to Pound-Drever-Hall laser frequency stabilization. Am. J. Phys., 69, 79, 2001.

[57] IntraAction Corp. Model AFM-802A1 Acousto-Optic Modulator Instruction Manual. Bellwood, IL, USA, 2010.

[58] B. E. A. Saleh and M. C. Teich. Fundamentals of Photonics. John Wiley \& Sons, Inc., Hoboken, New Jersey, USA, 2007.

[59] FastPulse Technology Inc. Series 5056 Self-Contained HV Switching Module QSwitch Driver. Saddle Brook, New Jersey, USA, 2004.

[60] E. A. Donley, T. P. Heavner, F. Levi, M. O. Tataw, and S. R. Jefferts. Double-pass acousto-optic modulator system. Rev. Sci. Instrum., 76, 063112, 2005.

[61] R. Steven Hargrove and Tehmau Kan. High power efficient dye amplifier pumped by copper vapor lasers. IEEE J. Quantum Electron., 16, 1108, 1980.

[62] C. Monroe, W. Swann, H. Robinson, and C. Wieman. Very cold trapped atoms in a vapor cell. Phys. Rev. Lett., 65, 1571, 1990.

[63] Steven Chu, L. Hollberg, J.E. Bjorkholm, Alex Cable, and A. Ashkin. Threedimensional viscous confinement and cooling of atoms by resonance radiation pressure. Phys. Rev. Lett., 55, 48, 1985. 
[64] Paul D. Lett, Richard N. Watts, Christoph I. Westbrook, William D. Phillips, Phillip L. Gould, and Harold J. Metcalf. Observation of Atoms Laser Cooled below the Doppler Limit. Phys. Rev. Lett., 61, 169, 1988.

[65] J. Dalibard and C. Cohen-Tannoudji. Laser cooling below the Doppler limit by polarization gradients: simple theoretical models. J Opt Soc Am B, 6, 2023, 1989.

[66] P. J. Ungar, D. S. Weiss, E. Riis, and Steven Chu. Optical molasses and multilevel atoms: theory. J Opt Soc Am B, 6, 2058, 1989.

[67] David S. Weiss, Erling Riis, Yaakov Shevy, P. Jeffery Ungar, and Steven Chu. Optical molasses and multilevel atoms: experiment. J Opt Soc Am B, 6, 2072, 1989.

[68] P. D. Lett, W. D. Phillips, S. L. Rolston, C. E. Tanner, R. N. Watts, and C. I. Westbrook. Optical molasses. J Opt Soc Am B, 6, 2084, 1989.

[69] Alan L. Migdall, John V. Prodan, William D. Phillips, Thomas H. Bergeman, and Harold J. Metcalf. First observation of magnetically trapped neutral atoms. Phys. Rev. Lett., 54, 2596, 1985.

[70] SAES Getters S. p. A. Alkali Metal Dispensers Brochure. Italy, 2007.

[71] T. Bergeman, Gidon Erez, and Harold J. Metcalf. Magnetostatic trapping fields for neutral atoms. Phys. Rev. A, 35, 1535, 1987.

[72] W. R. Anderson. PhD thesis, University of Virginia, 1996.

[73] H. Ludvigsen, A. Äijälä, A. Pietiläinen, H. Talvitie, and E. Ikonen. Laser cooling of rubidium atoms in a vapor cell. Phys. Scr., 49, 424, 1994. 
[74] W. Demtroder. Laser Spectroscopy: Basic Concepts and Instrumentation, Second Enlarged Edition. Springer-Verlag, Berlin, Germany, 1996.

[75] J. Han. PhD thesis, University of Virginia, 2009.

[76] Claude N. Cohen-Tannoudji. The Autler-Townes Effect Revisited, pages 109-123. Springer New York, New York, NY, 1996.

[77] F. Robicheaux, C. Wesdorp, and L. D. Noordam. Selective field ionization in Li and Rb: Theory and experiment. Phys. Rev. A, 62, 043404, 2000.

[78] Joseph Ladislas Wiza. Microchannel plate detectors. Nucl. Instruments Methods, 162, 587, 1979.

[79] Hewlett-Packard (Agilent Technologies). 6270B DC Power Supply Operating and Service Manual. Berkeley Heights, NJ, USA, 1970.

[80] Tektronix. AWG510 \& AWG520 Arbitrary Waveform Generator. Beaverton, OR, USA.

[81] Avtech Electrosystems LTD. Instructions: Model AV-141F. Ottawa, Ontario, Canada, 2013.

[82] Stanford Research Systems. Model DG535 Digital Delay/Pulse Generator. Sunnyvale, CA, USA, 2006.

[83] Stanford Research Systems. DG645 Digital Delay Generator. Sunnyvale, CA, USA, 2008. 
[84] G. C. Goodwin, S. F. Graebe, and M. E. Salgado. Control System Design. Pearson, USA, 2000.

[85] R Core Team. R: A Language and Environment for Statistical Computing. R Foundation for Statistical Computing, Vienna, Austria, 2016.

[86] Hadley Wickham and Romain Francois. dplyr: A Grammar of Data Manipulation, 2016. $\mathrm{R}$ package version 0.5.0.

[87] Hadley Wickham. tidyr: Easily Tidy Data with 'spread()' and 'gather()' Functions, 2016. R package version 0.6.0.

[88] Hadley Wickham, Jim Hester, and Romain Francois. readr: Read Tabular Data, 2016. R package version 1.0.0.

[89] Hadley Wickham, Romain Francois, and Kirill Müller. tibble: Simple Data Frames, 2016. R package version 1.2.

[90] Hadley Wickham. ggplot2: Elegant Graphics for Data Analysis. Springer-Verlag New York, 2009.

[91] Hadley Wickham. gtable: Arrange 'Grobs' in Tables, 2016. R package version 0.2.0.

[92] Jarek Tuszynski. caTools: Tools: moving window statistics, GIF, Base64, ROC AUC, etc., 2014. R package version 1.17.1.

[93] Robin K. S. Hankin. Special functions in r: introducing the gsl package. $R$ News, 6 , 2006. 
[94] Uwe Ligges et al. signal: Signal processing, 2014.

[95] Brian Richards. starkr: Calculation of Stark Maps for Rubidium-85, 2015. R package version 0.1.0.9000.

[96] T. Wilk, A. Gaëtan, C. Evellin, J. Wolters, Y. Miroshnychenko, P. Grangier, and A. Browaeys. Entanglement of two individual neutral atoms using Rydberg blockade. Phys. Rev. Lett., 104, 010502, 2010.

[97] Hendrik Weimer, Robert Löw, Tilman Pfau, and Hans Peter Büchler. Quantum critical behavior in strongly interacting Rydberg gases. Phys. Rev. Lett., 101, 250601, 2008.

[98] M. Saffman and K. Mølmer. Efficient multiparticle entanglement via asymmetric Rydberg blockade. Phys. Rev. Lett., 102, 240502, 2009.

[99] Thomas Amthor, Christian Giese, Christoph S. Hofmann, and Matthias Weidemüller. Evidence of antiblockade in an ultracold Rydberg gas. Phys. Rev. Lett., 104, 013001, 2010.

[100] Jens Honer, Hendrik Weimer, Tilman Pfau, and Hans Peter Büchler. Collective many-body interaction in Rydberg dressed atoms. Phys. Rev. Lett., 105, 160404, 2010.

[101] S. Sevinçli, N. Henkel, C. Ates, and T. Pohl. Nonlocal nonlinear optics in cold Rydberg gases. Phys. Rev. Lett., 107, 153001, 2011.

[102] D. D. Bhaktavatsala Rao and Klaus Mølmer. Dark entangled steady states of interacting Rydberg atoms. Phys. Rev. Lett., 111, 033606, 2013. 
[103] Matthew Ebert, Alexander Gill, Michael Gibbons, Xianli Zhang, Mark Saffman, and Thad G. Walker. Atomic fock state preparation using Rydberg blockade. Phys. Rev. Lett., 112, 043602, 2014.

[104] H. Gorniaczyk, C. Tresp, J. Schmidt, H. Fedder, and S. Hofferberth. Single-photon transistor mediated by interstate Rydberg interactions. Phys. Rev. Lett., 113, 053601, 2014.

[105] David Petrosyan and Klaus Mølmer. Binding potentials and interaction gates between microwave-dressed Rydberg atoms. Phys. Rev. Lett., 113, 123003, 2014.

[106] J. Pellegrino, R. Bourgain, S. Jennewein, Y. R. P. Sortais, A. Browaeys, S. D. Jenkins, and J. Ruostekoski. Observation of suppression of light scattering induced by dipoledipole interactions in a cold-atom ensemble. Phys. Rev. Lett., 113, 133602, 2014.

[107] Daniel Barredo, Henning Labuhn, Sylvain Ravets, Thierry Lahaye, Antoine Browaeys, and Charles S. Adams. Coherent excitation transfer in a spin chain of three Rydberg atoms. Phys. Rev. Lett., 114, 113002, 2015.

[108] Alexander W. Glaetzle, Marcello Dalmonte, Rejish Nath, Christian Gross, Immanuel Bloch, and Peter Zoller. Designing frustrated quantum magnets with laser-dressed Rydberg atoms. Phys. Rev. Lett., 114, 173002, 2015.

[109] R. M. W. Van Bijnen and T. Pohl. Quantum Magnetism and Topological Ordering via Rydberg Dressing near Förster Resonances. Phys. Rev. Lett., 114, 243002, 2015.

[110] F. Gounand, M. Hugon, P. R. Fournier, and J. Berlande. Superradiant cascading effects in rubidium Rydberg levels. J. Phys. B At. Mol. Phys., 12, 547, 1979. 
[111] C. Carr, R. Ritter, C. G. Wade, C. S. Adams, and K. J. Weatherill. Nonequilibrium phase transition in a dilute Rydberg ensemble. Phys. Rev. Lett., 111, 113901, 2013.

[112] R. H. Dicke. Coherence in Spontaneous Radiation Processes. Phys. Rev., 93, 99, 1954.

[113] N. Skribanowitz, I. P. Herman, J. C. MacGillivray, and M. S. Feld. Observation of Dicke superradiance in optically pumped HF gas. Phys. Rev. Lett., 30, 309, 1973.

[114] M. Gross, C. Fabre, P. Pillet, and S. Haroche. Observation of near-infrared Dicke superradiance on cascading transitions in atomic sodium. Phys. Rev. Lett., 36, 1035, 1976.

[115] D. Pavolini, A. Crubellier, P. Pillet, L. Cabaret, and S. Liberman. Experimental evidence for subradiance. Phys. Rev. Lett., 54, 1917, 1985.

[116] M. G. Moore and P. Meystre. Theory of Superradiant Scattering of Laser Light from Bose-Einstein Condensates. Phys. Rev. Lett., 83, 5202, 1999.

[117] J. I. Kim, R. B. B. Santos, and P. Nussenzveig. Manipulation of cold atomic collisions by cavity QED effects. Phys. Rev. Lett., 86, 1474, 2001.

[118] C. Greiner, B. Boggs, and T. W. Mossberg. Superradiant emission dynamics of an optically thin material sample in a short-decay-time optical cavity. Phys. Rev. Lett., 85, 3793, 2000.

[119] Chiu Fan Lee and Neil F. Johnson. First-order superradiant phase transitions in a multiqubit cavity system. Phys. Rev. Lett., 93, 083001, 2004. 
[120] Vasily V. Temnov and Ulrike Woggon. Superradiance and subradiance in an inhomogeneously broadened ensemble of two-level systems coupled to a low-Q cavity. Phys. Rev. Lett., 95, 243602, 2005.

[121] J. O. Day, E. Brekke, and T. G. Walker. Dynamics of low-density ultracold Rydberg gases. Phys. Rev. A, 77, 052712, 2008.

[122] Kirill Prozument, Anthony P. Colombo, Yan Zhou, G. Barratt Park, Vladimir S. Petrović, Stephen L. Coy, and Robert W. Field. Chirped-Pulse Millimeter-Wave Spectroscopy of Rydberg-Rydberg Transitions. Phys. Rev. Lett., 107, 143001, 2011.

[123] Anthony P. Colombo, Yan Zhou, Kirill Prozument, Stephen L. Coy, and Robert W. Field. Chirped-pulse millimeter-wave spectroscopy: Spectrum, dynamics, and manipulation of Rydberg-Rydberg transitions. J. Chem. Phys., 138, 014301, 2013.

[124] Florian Karlewski, Markus Mack, Jens Grimmel, Nóra Sándor, and József Fortágh. State-selective all-optical detection of Rydberg atoms. Phys. Rev. A, 91, 043422, 2015.

[125] Nicholas E. Rehler and Joseph H. Eberly. Superradiance. Phys. Rev. A, 3, 1735, 1971.

[126] R. Bonifacio and L. A. Lugiato. Cooperative radiation processes in two-level systems: Superfluorescence. Phys. Rev. A, 11, 1507, 1975.

[127] Zhi-Gang Feng, Lin-Jie Zhang, Jian-Ming Zhao, Chang-Yong Li, and Suo-Tang Jia. Lifetime measurement of ultracold caesium Rydberg states. J. Phys. B At. Mol. Phys., 42, 145303, 2009. 
[128] K. J. Weatherill, J. D. Pritchard, R. P. Abel, M. G. Bason, A. K. Mohapatra, and C. S. Adams. Electromagnetically induced transparency of an interacting cold Rydberg ensemble. J. Phys. B At. Mol. Opt. Phys., 41, 201002, 2008.

[129] Jianing Han and H. Maeda. Super-radiance-cascades and multimode super-radiance oscillations in a cold 85 Rb Rydberg gas. Can. J. Phys., 92, 1130, 2014.

[130] B. G. Richards and R. R. Jones. Dipole-dipole resonance line shapes in a cold Rydberg gas. Phys. Rev. A, 93, 042505, 2016.

[131] Wenhui Li, Michael W. Noel, Michael P. Robinson, Paul J. Tanner, Thomas F. Gallagher, Daniel Comparat, Bruno Laburthe Tolra, Nicolas Vanhaecke, Thibault Vogt, Nassim Zahzam, Pierre Pillet, and Duncan A. Tate. Evolution dynamics of a dense frozen Rydberg gas to plasma. Phys. Rev. A, 70, 042713, 2004.

[132] M. P. Robinson. PhD thesis, University of Virginia, 2002.

[133] M. Gross and S. Haroche. Superradiance: An essay on the theory of collective spontaneous emission. Phys. Rep., 93, 301, 1982.

[134] M. R. Kutteruf and R. R. Jones. Controlled dipole-dipole interactions between K Rydberg atoms in a laser-chopped effusive beam. Phys. Rev. A, 82, 063409, 2010.

[135] Myron L. Zimmerman, Michael G. Littman, Michael M. Kash, and Daniel Kleppner. Stark structure of the Rydberg states of alkali-metal atoms. Phys. Rev. A, 20, 2251, 1979. 
[136] Nicolas Saquet, Anne Cournol, Jérôme Beugnon, Jacques Robert, Pierre Pillet, and Nicolas Vanhaecke. Landau-Zener transitions in frozen pairs of Rydberg atoms. Phys. Rev. Lett., 104, 133003, 2010.

[137] P. Hertz. Math. Ann., 67, 1909.

[138] M. Abramowitz and I. Stegun. Handbook of Mathematical Functions with Formulas, Graphs, and Mathematical Tables. Dover Publications, New York, USA, 1964.

[139] J. B. Balewski, A. T. Krupp, A. Gaj, S. Hofferberth, R. Löw, and T. Pfau. Rydberg dressing: Understanding of collective many-body effects and implications for experiments. New J. Phys., 16, 063012, 2014.

[140] G. Pupillo, A. Micheli, M. Boninsegni, I. Lesanovsky, and P. Zoller. Strongly correlated gases of Rydberg-dressed atoms: Quantum and classical dynamics. Phys. Rev. Lett., 104, 223002, 2010.

[141] C. Gaul, B. J. DeSalvo, J. A. Aman, F. B. Dunning, T. C. Killian, and T. Pohl. Resonant Rydberg Dressing of Alkaline-Earth Atoms via Electromagnetically Induced Transparency. Phys. Rev. Lett., 116, 243001, 2016.

[142] S. Wüster, C. Ates, A. Eisfeld, and J. M. Rost. Excitation transport through Rydberg dressing. New J. Phys., 13, 073044, 2011.

[143] Wenhui Li, Paul J. Tanner, and T. F. Gallagher. Dipole-Dipole Excitation and Ionization in an Ultracold Gas of Rydberg Atoms. Phys. Rev. Lett., 94, 173001, 2005. 